\title{
SSC/SSCS SSP/SSTS Joint Annual Meeting 2020
}

Davos, Switzerland, 3-5 June, 2020

(The meeting has been cancelled due to COVID-19) 


\section{Obstructive Lung Disease, Epidemiology, Health Prevention}

\section{1 \\ Experimental Dyspnea Interferes with Locomotion and Cognition: A Randomised Trial}

D. Lawi ${ }^{1}$, E. Dupuis-Lozeron
T. Similowski, , G. Berra
1, G. G. Allali, ${ }^{1,2,3}$,

${ }^{1}$ Hôpitaux Universitaires de Genève, Genève, ${ }^{2}$ University of Geneva, Geneva, Switzerland, ${ }^{3}$ Department of Neurology, Albert Einstein College of Medicine, New York, NY, United States, ${ }^{4}$ Groupe Hospitalier Pitié-Salpêtrière Charles Foix, ${ }^{5}$ Sorbonne University, Paris, France

Background: Chronic respiratory diseases are associated with cognitive dysfunction, but whether dyspnea by itself negatively impacts on cognition has not been demonstrated. Cortical networks engaged in subjects experiencing dyspnea are also activated during other tasks that require cognitive input and may provoke a negative impact through interference with each other.

Methods: This crossover randomised trial investigated whether experimentally-induced dyspnea would negatively impact on locomotion and cognitive function among 40 healthy adults. Crossover conditions were unloaded breathing or loaded breathing using an inspiratory threshold load. To evaluate locomotion, participants were assessed by the Timed Up and Go test. Cognitive function was assessed by categorical and phonemic verbal fluency tests, the Trail Making Test A and B (executive function), the CODE test from the WAIS-IV (processing speed), and by direct and indirect digit span (working memory).

Results: The mean time difference to perform the Timed Up and Go test between unloaded and loaded breathing was $-0.75 \mathrm{sec}$ $(-1.01$ to $-0.49 ; \mathrm{p}<0.001)$. Executive function, processing speed and working memory performed better during unloaded breathing, particularly for subjects starting first with the loaded breathing condition.

Conclusion: Our data suggest that respiratory threshold loading to elicit dyspnea had a major impact on locomotion and cognitive function in healthy adults.

Disclosure: Nothing to disclose

Table 1. Baseline characteristics of participants (for Abstract 1)

\begin{tabular}{|c|c|c|c|}
\hline Characteristics $^{\star}$ & $\begin{array}{l}\text { Unloaded } \\
\text { breathing first } \\
(\mathrm{n}=20)\end{array}$ & $\begin{array}{l}\text { Loaded } \\
\text { breathing first } \\
(\mathrm{n}=20)\end{array}$ & Total $(n=40)$ \\
\hline Age - yr & $26.35 \pm 4.94$ & $26.60 \pm 5.14$ & $26.48 \pm 4.98$ \\
\hline Male sex - n (\%) & $11(55)$ & $13(65)$ & $24(60)$ \\
\hline $\mathrm{BMI}-\mathrm{kg} / \mathrm{m} 2$ & $23.50 \pm 4.00$ & $22.98 \pm 3.73$ & $23.24 \pm 3.82$ \\
\hline $\begin{array}{l}\text { FEV1/FVC - } \\
\text { predicted \% }\end{array}$ & $101.15 \pm 6.23$ & $102.45 \pm 8.20$ & $101.80 \pm 7.22$ \\
\hline $\begin{array}{l}\text { FEV1 - } \\
\text { predicted \% }\end{array}$ & $100.40 \pm 11.25$ & $101.30 \pm 12.45$ & $100.85 \pm 11.72$ \\
\hline MIP - cmH20 & $111.65 \pm 24.34$ & $106.65 \pm 25.86$ & $109.15 \pm 24.92$ \\
\hline SNIP - cmH20 & $104.00 \pm 25.02$ & $86.75 \pm 20.82$ & $95.38 \pm 24.34$ \\
\hline $\begin{array}{l}\text { Inspiratory } \\
\text { load } \dagger \text { - level }\end{array}$ & $5.92 \pm 2.28$ & $4.33 \pm 2.52$ & $5.12 \pm 2.51$ \\
\hline
\end{tabular}




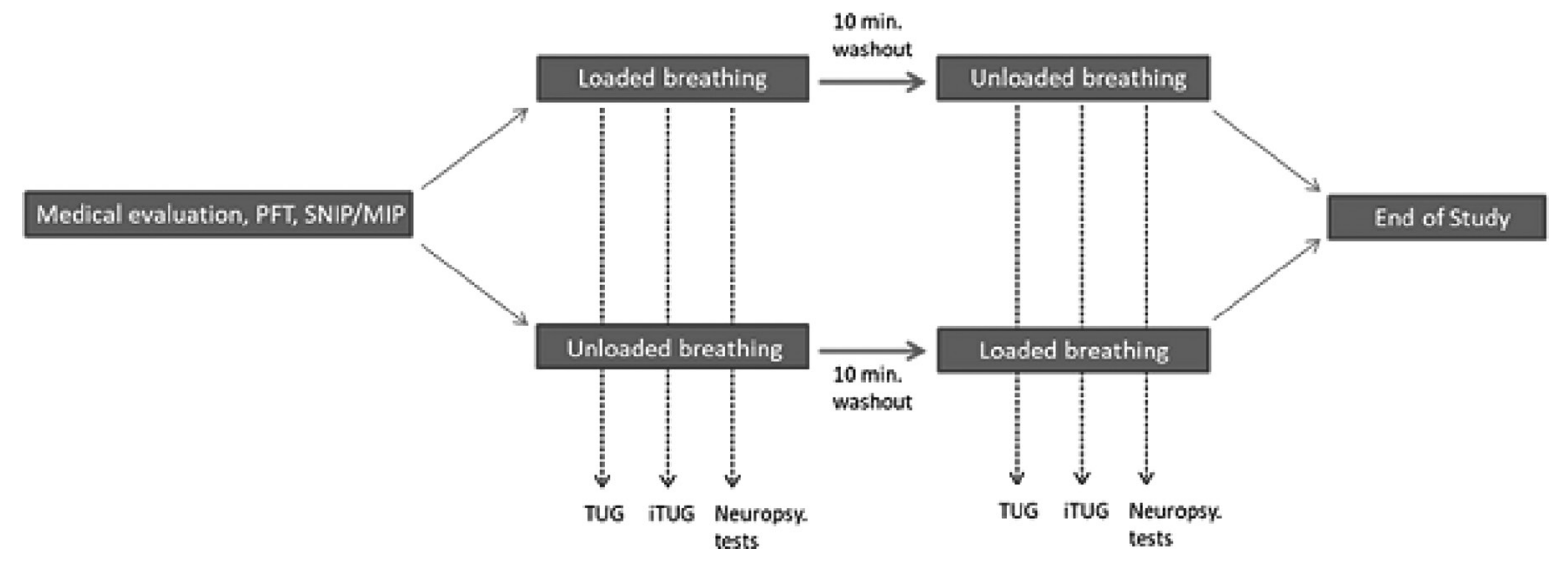

Fig. 1. Study design ${ }^{*}$ Randomized, open label, two-condition, two-period crossover design (for Abstract 1).

Fig. 2. TUG test performance. A: overall effect; B:Classified by experimental sequence (for Abstract 1).
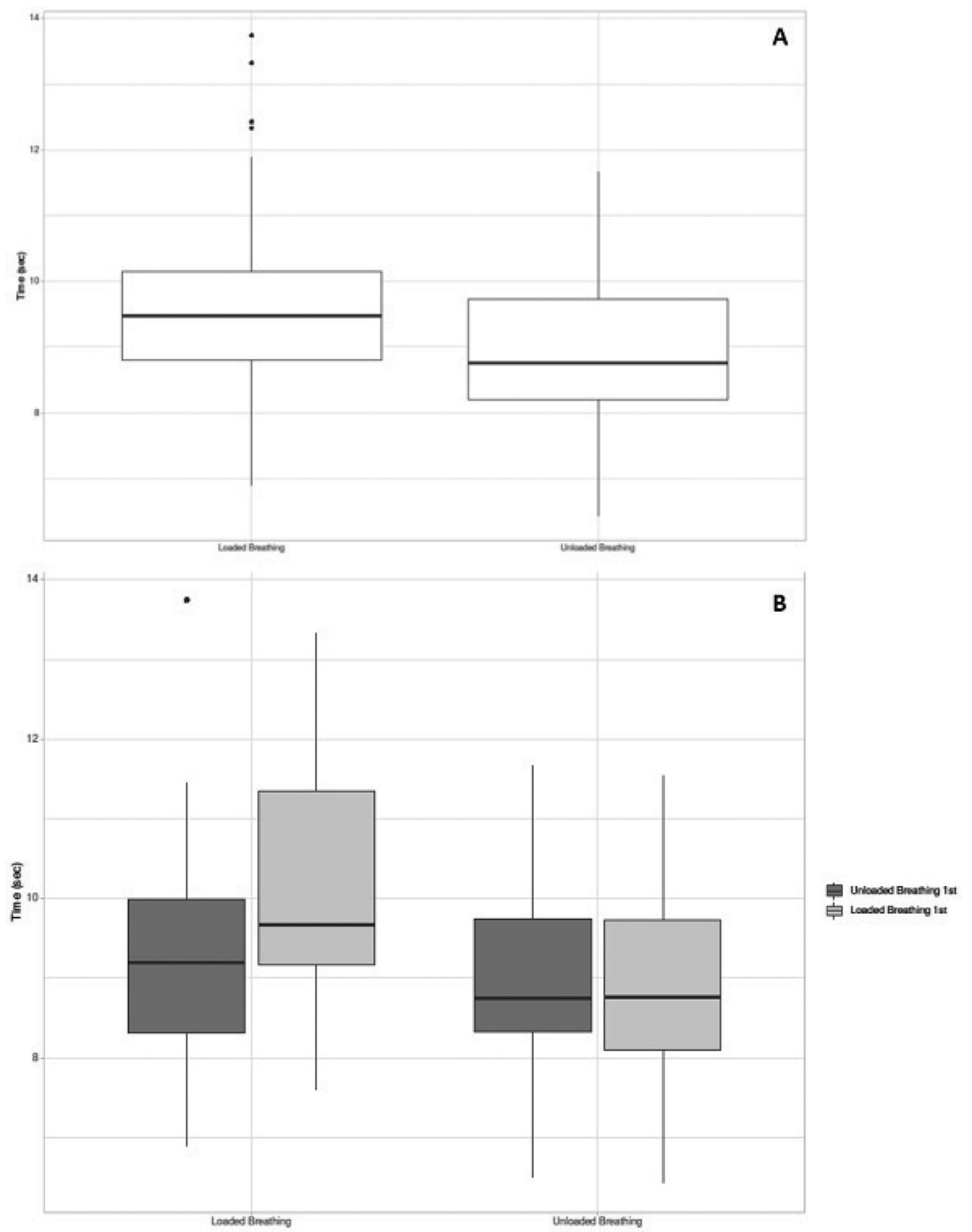


\section{5}

\section{Smartphone-Based Cough Detection Predicts Asthma Control - Description of a Novel, Scalable Digital Biomarker}

F. Rassouli ${ }^{1}$, P. Tinschert ${ }^{2}$, F. Barata ${ }^{3}$, C. Steurer-Stey ${ }^{4}$, E. Fleisch ${ }^{2,3}$, M. Puhan ${ }^{4}$, T. Kowatsch ${ }^{2,3}$, M. Brutsche 1

${ }^{1}$ Lung Center, Cantonal Hospital St. Gallen, ${ }^{2}$ University of St. Gallen, St. Gallen, ${ }^{3}$ ETH Zurich, ${ }^{4}$ University of Zurich, Zurich, Switzerland

Introduction: The frequency of nocturnal cough might be a predictor for asthma control. However, information regarding longitudinal trends and cause-effect relationships of nocturnal cough in asthma is lacking. This study aimed to investigate nocturnal cough in asthmatic individuals over the course of 4 weeks by means of a smartphone and to explore the interplay between nocturnal cough frequency and asthma control.

Methods: Machine learning models for nocturnal cough detection were developed. 94 patients in 2 centers were recruited; study duration per patient was 29 days with in-person appointments on first and last study day. In between, patient-reported outcomes and nocturnal sensor data were collected by a smartphone with a chatbased study app. Asthma control was assessed by weekly ACT (Asthma Control Test). Separate mixed effect models were calculated to understand whether nocturnal cough could predict asthma control.

Results: The characteristics of nocturnal cough are presented in a separate abstract. The mixed effect model demonstrates that weekly aggregated measurements of nocturnal cough were associated with asthma control of the same and the subsequent week. An increase of 100 coughs per week was associated with a decrease of 0.56 points in ACT for this week $(\mathrm{p}=0)$ and a decrease of 0.25 points in ACT for the following week ( $\mathrm{p}=0.024)$.

Conclusion: Our study is the first to describe the independent predictive value of changes of nocturnal cough frequency for asthma control. Nocturnal cough could therefore be a useful parameter for timely medical interventions to prevent asthma deteriorations. By using conventional smartphones, this study lays the groundwork for scalable, validated and broadly available digital biomarkers for asthma.

Disclosure: Nothing to disclose

\section{9}

The Anatomy of Asthma-Related Nocturnal Cough A Potential New Digital Biomarker

\author{
F. Rassouli ${ }^{1}$, P. Tinschert ${ }^{2}$, F. Barata ${ }^{3}$, C. Steurer-Stey ${ }^{4}$, E. Fleisch ${ }^{2,3}$, \\ M. Puhan ${ }^{4}$, T. Kowatsch ${ }^{2,3}$, M. Brutsche ${ }^{1}$ \\ ${ }^{1}$ Lung Center, Cantonal Hospital St. Gallen, ${ }^{2}$ University of St. \\ Gallen, St. Gallen, ${ }^{3}$ ETH Zurich, ${ }^{4}$ University of Zurich, Zurich, \\ Switzerland
}

Introduction: Nocturnal cough in asthma is not well-defined. The frequency of nocturnal cough might be a valid marker for asthma control, however, information regarding longitudinal trends and cause-effect relationships of nocturnal cough in asthma is lacking. This study aimed to investigate prevalence, trends and characteristics of nocturnal cough in asthmatic individuals over the course of 4 weeks.

Methods: Machine learning models for nocturnal cough detection were developed. 94 patients in 2 centers were recruited; study duration per patient was 29 days with in-person appointments on first and last study day. In between, patient-reported outcomes and nocturnal sensor data were collected by a smartphone with a chatbased study app.

Results: Patients coughed in 53\% of 1181 nights (range 0-345 coughs/night). Figure 1 shows nocturnal cough rates per patient, showing considerable variance between patients. The highest counts were measured in the first 30 minutes in bed (4.5-fold higher than rest of night, Figure 2). Mean cough latency was 29 minutes (range $0-553$ ), 86\% of coughs were part of a cough cluster. Clusters consisted of a median of 2 coughs (IQR 2-4). Median coughs per hour were 0 (IQR 0-1). Nocturnal cough was persistent within patient up to all 28 nights.

Conclusion: Our study is the first to describe prevalence, longitudinal trends and characteristics of nocturnal cough in asthma over a period of one month, demonstrating that it was a prevalent symptom with huge variance between patients but high persistence within patients. Cough events were $4.5 \mathrm{x}$ more frequent within the first 30 minutes of bedtime indicating a potential role of positional change. Nocturnal cough characteristic could serve

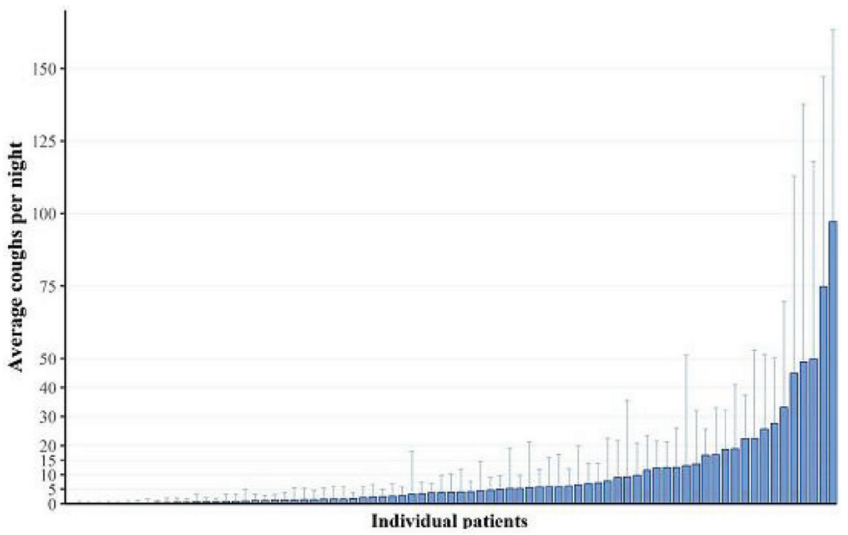

Fig. 1. Nocturnal cough counts - each bar represents one patient (for Abstract 19). 
Fig. 2. Distribution of cough events in relation to bedtime (log-scaled) (for Abstract 19).

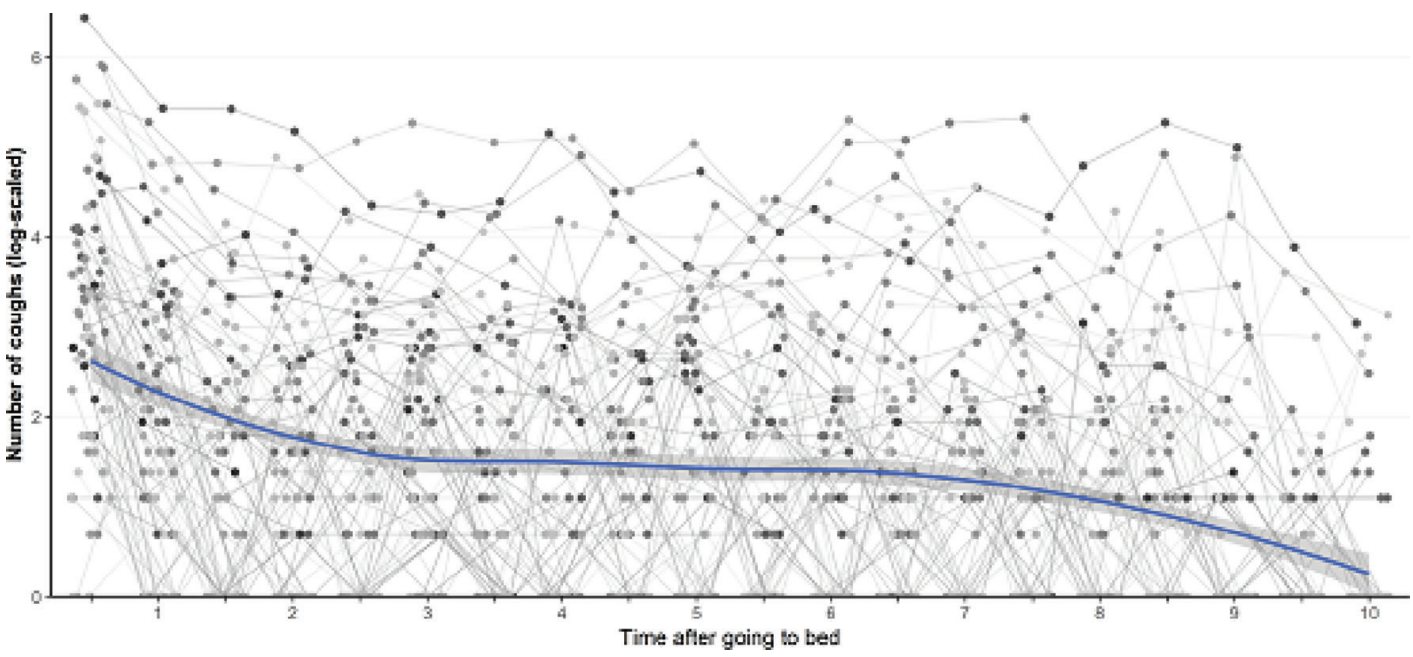

to generate hypotheses regarding pathophysiological aspects of cough. One next step will be the investigation of the association of nocturnal cough and asthma control.

Disclosure: Nothing to disclose

\section{3 \\ Outpatient Pulmonary Rehabilitation in Copd Patients: A 12 Years Single Centre Experience}

\section{S. Rezek', M. Hofer ${ }^{2}$, G. Mungo' , T. Hess' ${ }^{2}$, S. Beyer ${ }^{2}$ \\ ${ }^{1}$ Physiotherapie, ${ }^{2}$ Pneumologie, Kantonsspital Winterthur, Winterthur, Switzerland}

Introduction: In 2008 the departments of pneumology and physiotherapy of the Cantonal Hospital Winterthur established an outpatient pulmonary rehabilitation program (APR). Patients with chronic lung diseases mostly with COPD, exercised 3-times weekly for 3 months according to a program based on international guidelines. Our aim was to evaluate functional capacity and quality of life over the 12-year period in our APR.
Methods: The following outcomes were retrospectively analysed in the included COPD-APR-patients: FEV1, 6 minutes walk test (6MWT) and chronic respiratory questionnaire (CRQ). Outcome comparison of the following groups:

- 83 patients with mild to moderate (COPD $1 / 2$ ) to those 83 with severe to very severe obstruction (COPD 3/4)

- 26 patients with a second APR cycle to the first APR

- 87 (47\%) patients within the first (early) and the 99 (53) within the last 6 years (late).

Results: Since 2008424 Patients were included. 271 Patients (64\%) suffered from COPD of whom 199 (73\%) finished the program and from 186 all data was available. The demographics are the following: $112(60 \%)$ men, mean age 67.1 years $(95 \% \mathrm{CI}$ 65.5-68.8), FEV1 1.32 litre (1.24-1.40), 52\% predicted (49-54), distribution of COPD Grade 1 to 4 of 14 (8\%), 79 (42\%), 73 (39\%) and $20(11 \%)$ respectively.

There was no significant difference of $\triangle 6 \mathrm{MWT}(21 ; 9.2-32.5 \mathrm{vs}$. $32 ; 21-44)$ and $\triangle$ CRQ (1.8;1.2-2.3 vs. $1.3 ; 0.6-2.1)$ in COPD $1 / 2$ compared to COPD $3 / 4$. $\triangle$ CRQ was the same in the early and the late group ( $\triangle \mathrm{CRQ}$ total $1.4 ; 0.7-2.2$ vs. $1.7 ; 1.0-2.3)$; nevertheless, there was a significant improvement of $\triangle 6 \mathrm{MWT}$ in the late group (39; $28-50$ vs. 12 ; 0-24 meters) with no difference in FEV1\% at

Table 1. Main Results (for Abstract 23)

\begin{tabular}{lccc}
\hline Parameter & Pre APR & Post APR & Delta \\
\hline 6MWT (meter) & $412(397-427)$ & $438(422-455)$ & $27(18-35)^{*} \wedge$ \\
CRQ Dyspnoea & $4.2(4.0-4.4)$ & $4.6(4.4-4.8)$ & $0.4(0.3-0.6)^{\star} \wedge$ \\
CRQ Fatigue & $4.6(4.4-4.8)$ & $4.8(4.7-5.0)$ & $0.3(0.1-0.4)^{*} \wedge$ \\
CRQ Emotion & $4.9(4.7-5.0)$ & $5.2(5.0-5.4)$ & $0.3(0.2-0.5)^{*} \wedge$ \\
CRQ Mastery & $5.0(4.8-5.2)$ & $5.5(5.3-5.7)$ & $0.5(0.3-0.6)^{*} \wedge$ \\
CRQ Total & $18.7(17.9-19.3)$ & $20.1(19.6-20.8)$ & $1.5(1.1-2.0)^{*} \wedge$
\end{tabular}

${ }^{*} \mathrm{p}<0.001 \wedge \geq \operatorname{MCID}$ (minimal clinical important difference). 
inclusion. The 26 patients with a second APR cycle showed the same improvement in $\triangle 6 \mathrm{MWT}(37 ; 12-63$ vs. $35 ; 10-61)$ and $\Delta$ CRQ ( $\triangle$ CRQ total $1.7 ; 0.1-3.3$ vs. $2.5 ; 0.9-4.1$ ) compared to the first cycle.

Conclusions: We found clinical meaningful improvement in $6 \mathrm{MWT}$ and quality of live (measured with CRQ) in our APR comparable to reported results in the literature. In our APR there was no difference of improvement in CRQ and 6MWT in mild to moderate compared to severe to very severe COPD patients. Nevertheless, in the later phase of the program (second 6 years) there was an improvement of $\triangle 6 \mathrm{MWT}$, but not in $\triangle \mathrm{CRQ}$ with comparable COPD-grade. A second cycle of APR showed the same improvement in 6MWT and CRQ as the first.

Disclosure: Nothing to disclose

\section{2 \\ Sexuality in Chronic Obstructive Pulmonary Disease (Sexy Copd)

\author{
C. Steurer-Stey ${ }^{1,2}$, A. Strassmann ${ }^{1}$, K. Dalla Lana ${ }^{1}$, J. Gauer ${ }^{2}$, A. Frei ${ }^{1}$, \\ M. Puhan ${ }^{1}$ \\ ${ }^{1}$ Epidemiology, Biostatistics and Prevention Institute, University \\ of Zurich, ${ }^{2}$ mediX Gruppenpraxis Zurich, Zurich, Switzerland
}

Introduction: Sexuality, which is a determinant of quality of life, is often ignored and under-researched in patients with COPD.

Methods: We developed, piloted and launched one questionnaire for COPD patients and one for health care professionals asking them about their attitudes, actions, experiences, barriers and enablers when talking (or not) about sexuality.

Results: 22 COPD patients from primary care (mean age 62.9 a, $50 \%$ female, mMRC $2.1 \pm 1.3,61 \%$ in a relationship) and 36 health care professionals (mean age $45.7,56 \%$ female, GPs $33 \%$, lung specialists $19 \%$, nurses/physiotherapists $47 \%$ ) answered the questionnaires. $83 \%$ of patients reported that they have never been asked about sexuality since COPD diagnosis and $89 \%$ did not bring up the topic themselves. $33 \%$ wanted to be directly approached by health care professionals and $50 \%$ preferred raising the issue themselves. $40 \%$ thought that health care professionals might not feel comfortable with the topic. For $72 \%$ of patients a bond of trust was the most important enabler to talk about sexuality.

Fig. 1. Remote Monitoring of Physical Activity and CAT Score in COPD Patients (for Abstract 54).

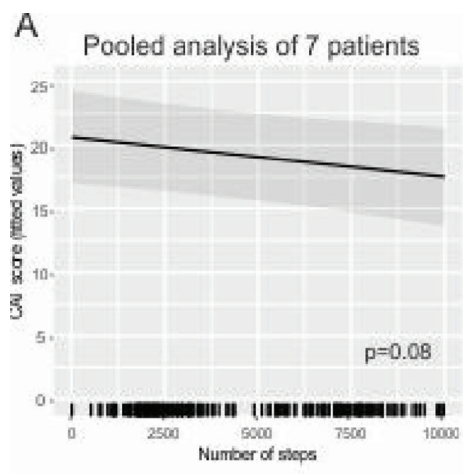

$86 \%$ of health care professionals stated sexuality as being important for quality of life. $69 \%$ wanted to talk about sexuality, but $49 \%$ never and $46 \%$ only very rarely did. Main barriers were not to know how to start the conversation and the assumption that COPD patients feel ashamed and overstrained or might have religious and cultural reluctance which was rebutted by the patients' answers.

Conclusions: There is a patient need and simultaneously a misconception and lack in self-confidence among health care professionals to talk about sexuality. It is time to include communication about sexuality in COPD care. The development of disease-specific instruments could facilitate to talk about it and to include the assessment of sexuality into practice and outcome research.

Disclosure: Nothing to disclose

\section{4 \\ Tracking Physical Activity of COPD Patients in a Real-World Setting}

\section{Bösch, F. Rassouli, V. Filipin-Horvath, S. Widmer, F. Baty,} M.H. Brutsche

Lung Center, Cantonal Hospital St. Gallen, St.Gallen, Switzerland

Introduction: Chronic obstructive pulmonary disease (COPD) is a highly prevalent disease with rising incidence worldwide. The main symptom of the disease is exertional dyspnea due to progressive and irreversible airflow obstruction, with an associated decrease of physical activity (PA). Monitoring PA may help to improve the clinical management of COPD and open new avenues for early intervention.

Methods: In this monocentric, prospective, observational cohort study of 85 patients with COPD stage GOLD B or higher (NCT03855670), we remotely monitor the PA of participating patients in a real-world setting over the period of one year using a commercially available activity-tracking device (Garmin Vivofit 3 ). Activity data are synchronized to a smartphone app via Bluetooth and sent to the investigators using a specially designed computer interface. The readout is complemented, amongst others, by a weekly COPD assessment test (CAT) as well as semiannual bioelectrical impedance- and blood analyses.

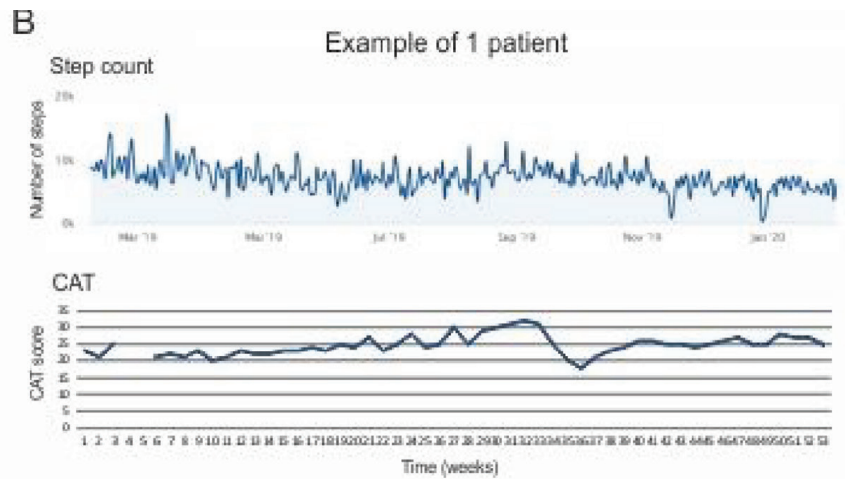


Results: Up to now, 31 patients are enrolled in the trial of which 7 have already completed the study. Remote monitoring of PA in COPD patients with activity trackers is feasible, and there is a high interpatient variability of PA. Preliminary data from the 7 completed patients suggest that the level of PA may correlate with intraindividual CAT score changes (Figure 1). Whether acute exacerbations of COPD correlate with characteristic declines in PA remains to be analyzed when more patients have completed the study.

Conclusion: Remote tracking of the PA in patients with COPD is feasible and allows assessing an important, functional qualityof-life marker in a real-world setting. Extrapolation of PA tracking to future interventional COPD trials will be crucial to get a more comprehensive picture of overall patient benefit. Considering the typically higher age of COPD patients, user friendliness of activitytracking devices is an important factor to consider.

Disclosure: Nothing to disclose

\section{5}

\section{Telehealth vs. Standard Care in Copd - A Randomized Controlled Trial}

\begin{abstract}
F. Rassouli ${ }^{1}$, F. Baty ${ }^{1}$, M. Kohler ${ }^{2}$, D. Stolz ${ }^{3}$, R. Thurnheer ${ }^{4}$, T. Brack ${ }^{5}$, C. Kähler ${ }^{6}$, S. Widmer ${ }^{1}$, U. Tschirren ${ }^{1}$, N.A. Sievi ${ }^{2}$, M. Tamm ${ }^{3}$, M.H. Brutsche

${ }^{1}$ Lung Center, Cantonal Hospital St. Gallen, St. Gallen, ${ }^{2}$ Clinic for Pulmonology, University Hospital Zurich, Zurich, ${ }^{3} \mathrm{Clinic}$ for Pulmonology, University Hospital Basel, Basel, ${ }^{4} \mathrm{Clinic}$ for Internal Medicine, Cantonal Hospital Münsterlingen, Münsterlingen, ${ }^{5} \mathrm{Clinic}$ for Internal Medicine, Cantonal Hospital Glarus, Glarus, Switzerland, ${ }^{6}$ Clinic for Pulmonology, WaldburgZeil-Kliniken, Wangen, Germany
\end{abstract}

Introduction: Different studies investigating telehealth care (TC) for COPD have shown conflicting results. In a feasibility trial we showed excellent patient adherence and satisfaction with our TCapproach ${ }^{1}$. Here, results of a consecutive randomized controlled trial are presented.

Methods: Patients were randomly assigned to either TC or standard care (SC) with a crossover after 6 months. During TC patients answered 6 daily questions online, focused on the early recognition of exacerbations (AECOPD) and a weekly COPD assessment test (CAT). Entries were followed during working days with a pre-specified action plan. During SC patients received standard care without TC. The slope of individual CAT changes over the study periods were calculated. The primary endpoint was the group-specific slope difference of weekly CAT over time. Satisfaction with care was assessed by a visual analogue scale from 0-10.

Results: In total, 165 of 175 patients (94\%) completed the trial. The overall mean CAT score was 15.6 points. The mean CAT increase was 3.6 vs. 1.8 points/year in the $\mathrm{SC}$ and TC groups respectively $(\mathrm{p}=0.0015$; Figure 1$)$. Satisfaction with care at baseline was 8.2 , at the end of SC $8.5(\mathrm{p}=0.062)$ and after TC $8.8(\mathrm{p}=0.036)$. The main results of important secondary endpoints are summarised in Table 1.

Conclusion: While receiving TC the slope of the CAT increase an indicator of the naturally progressive course of COPD - was significantly reduced by $50 \%$ ( -1.8 points/year). Satisfaction with care - already high before the intervention - increased further with TC. With TC, we detected a higher number of moderate AECOPD, probably indicating a higher diagnostic sensitivity than without TC. TC was associated with a trend to fewer days in hospital and ower total COPD-related costs.

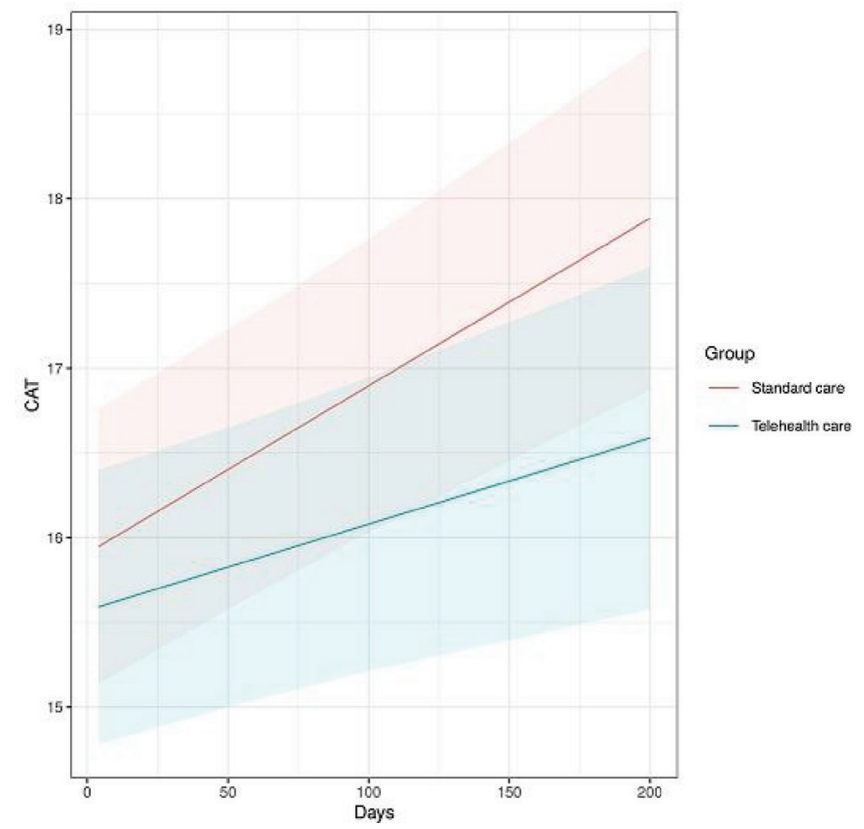

Fig. 1. CAT increase over time (for Abstract 75).

Table 1. Secondary endpoints (main results) (for Abstract 75)

\begin{tabular}{lccc}
\hline & Telehealth care & Standard care & p \\
\hline ED visit rate due to AECOPD & $0.47 /$ year [95\%CI: $0.37-0.59]$ & $0.62 /$ year [95\%CI: $0.5-0.75]$ & 0.140 \\
Hospitalisation rate due to AECOPD & 0.17/year [95\%CI: $0.11-0.25]$ & $0.17 /$ year [95\%CI: 0.11-0.25] & 0.618 \\
Number of AECOPD (total) & 125 & 99 & 0.060 \\
Number of moderate AECOPD & 99 & 72 & 0.028 \\
Days in hospital due to AECOPD (total) & 187 & 282 & 0.497 \\
COPD-related costs & $2587 \mathrm{CHF} / \mathrm{py}$ & $4240 \mathrm{CHF} / \mathrm{py}$ & 0.364 \\
\hline
\end{tabular}


References: 1. Rassouli $\mathrm{F}$ et al. Telehealthcare for Chronic Obstructive Pulmonary Disease in Switzerland Is Feasible and Appreciated by Patients. Respiration. 2016;92(2):107-13.

Disclosure: Nothing to disclose

139

CAir: Quality-of-Life Management for Copd and Asthma Patients - A Usability Study

\author{
D. Kohlbrenner ${ }^{1}$, C.S. Gross² , A. Asisof ${ }^{2}$, M. Nissen² , A. Ivankay 3 , \\ T. Brunschwiler', C.F. Clarenbach ${ }^{1}$ \\ ${ }^{1}$ Department of Pulmonology, University Hospital Zurich, \\ ${ }^{2}$ Department of Management, Technology and Economics, \\ ETH Zurich, Zurich, ${ }^{3}$ Cognitive Computing \& Industry Solutions, \\ ${ }^{4}$ Science \& Technology, IBM Research, Rueschlikon, Switzerland
}

Introduction: Several disease management programmes for COPD and Asthma are available and effective. Actual research focuses on providing these interventions to a broad spectrum of patients, while keeping costs low. Home-systems allow selfmeasurement and automated data transfer to healthcare providers. To our knowledge, no comprehensive home-system is available in the field of respiratory medicine.

Our aim was to investigate the usability of the newly designed CAir-desk in patients with COPD and Asthma. Primary focus was on device functionality and operability, data transfer and storage, as well as patient compliance.

Methods: The CAir-desk is a custom-built system, consisting of a smartphone (including symptom burden questionnaires and cough-recording), spirometer, physical activity tracker, and an airquality monitor.

We conducted a usability study, investigating the CAir-desk in a sample of 10 patients.

Results: We present preliminary data from assessing a sample of COPD and Asthma patients using the CAir-desk for 1 month. Technical performance, data transfer and storage performed well. Acceptance and adherence were diverse and differed between the two patient groups.

Conclusion: The CAir-desk performed technically well in this usability study. Acceptance rates reported by the study participants will influence the planning of a subsequent randomized controlled trial. Chronic respiratory diseases such as COPD and Asthma require both long-term health care. Technological advances, including "hospital at home" strategies, will challenge traditional care models in the future.

Disclosure: Nothing to disclose

\section{0 Prescription Behaviour of Short-Acting B2-Agonists in Switzerland}

C. Schuoler
V. Chicherov

${ }^{1}$ Medical Department, AstraZeneca AG, Baar, ${ }^{2}$ Epidemiology, Biostatistics and Prevention Institute, University of Zurich, Zurich, ${ }^{3}$ Lung Center Bern, Salem Hospital, Bern, ${ }^{4}$ Internal Medicine, Sierre, ${ }^{5} \mathrm{IQV} I \mathrm{~A}$ Solutions $\mathrm{GmbH}$, Rotkreuz, Switzerland

Introduction: Due to safety concerns GINA (Global Initiative for Asthma) no longer recommends SABA (short-acting $\beta_{2}$-agonist) monotherapy, but a combination of inhaled corticosteroids (ICS)-formoterol (a fast as well as long-acting $\beta_{2}$-agonist) as preferred reliever option across all asthma severities and for the treatment of asthma. SABA overreliance and overuse has been reported to be a serious issue in some countries, however, no such data are available for Switzerland.

Methods: This is a retrospective study of anonymized patient level prescription data from Swiss pharmacies (IQVIA Swiss pharmacy LRx database) during the period of November 2016 to October 2019 (3 years). Asthma patients were classified based on patient gender, age and historic medication by a predictive model trained on prescription data with diagnoses (IQVIA Disease Analyzer ) from IQVIA Germany.

Results: In the given timeframe 8.145 asthma patients (age > 4 years) with at least one asthma specific prescription per year were identified. In the period from October 2018 to October 2019 14\% of patients had exclusively a SABA prescription, 38\% SABA together with another asthma specific medication $(\mathrm{SABA}+)$ and $48 \%$ had another medication (Fig. 1). $75 \%$ of children (4-11 years) had a SABA prescribed. Within the SABA only users $37 \%$ had three or more canisters per year prescribed, in the SABA+ group $41 \%$, respectively.

Conclusion: Although SABA prescription in Switzerland is moderate, a considerable number of patients tend to over-rely on their medication ( $\geq 3$ canisters/year). The fact that SABA and $\mathrm{SABA}+$ patients exhibit a similar pattern of extensive SABA prescription points to an urgent need of behavioural change when asthma therapy is intensified requiring anti-inflammatory treatment. Closed fixed combinations of ICS/formoterol as initial asthma therapy according to GINA could ameliorate this problem, whereas future over the counter distribution of SABA could aggravate overuse.

Disclosure: Claudia Steurer-Stey: advisory boards and speaker for AstraZeneca, GlaxoSmithKlein, Novartis, Boehringer Ingelheim; Elke Ullmer, Romeo Providoli: advisory boards for AstraZeneca; Vitaly Chicherov, Maria Korobeynikova and Stefan Wetzel are employees of IQVIA, which conducted the presented work under contract for AstraZeneca; Claudio Schuoler and Gunther Pendl are employees of AstraZeneca 


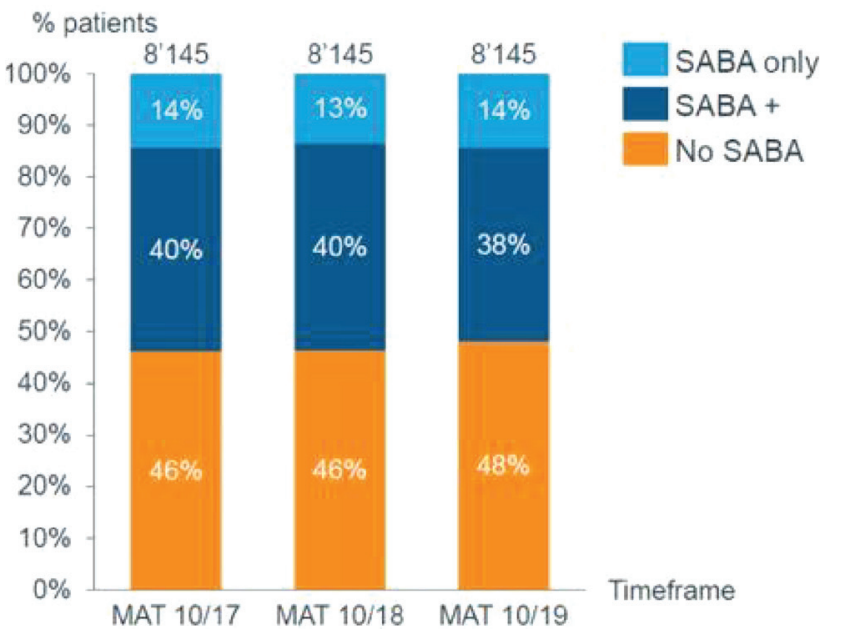

Fig. 1. SABA prescriptions in Switzerland; MAT=Moving Annual Total (for Abstract 140).

\section{1 \\ Visualization and Pattern Detection in the Dynamic of Smoking Lapse and Relapse during Smoking Cessation}

\section{Paciorkowski, F. Baty, M. Brutsche}

Cantonal Hospital St. Gallen, St. Gallen, Switzerland

Introduction: Smoking cessation is a priority of public health. The rate of relapse in smokers attempting to quit is generally high and it is of interest to better understand the dynamic of lapse and relapse during smoking cessation. Using exploratory statistical techniques combined with novel visualization tools, we aimed to characterize relapsing patterns and investigate their impact on long-term abstinence.

Methods: In this retrospective study, data were collected from 959 participants entering our in- and out-patient smoking cessation program between March 2012 and January 2015. Data consisted of baseline information (demographics, smoking history and dependence level) and longitudinal data about tobacco consumption. Features were extracted from the patient's lapse/ relapse sequence and analyzed using principal component analysis (PCA). Analytical and visualization tools from the R statistical software were used.

Results: Baseline characteristics of the study participants are summarized in Table 1. The proportion of patients who were continuously abstinent, never quit or relapsed with intermittence during their follow-up was $23 \%, 40 \%$ and $37 \%$, respectively. The dynamic of lapse and relapse among intermittent patients showed various behavioral patterns. Patients could be clustered into three main groups (Figure 1): patients with prolonged abstinence over a long follow-up (cluster 1), patients with frequent relapses over an

Table 1. Patients baseline characteristics (for Abstract 151)

$\begin{array}{ll}\text { Number of patients, } \mathrm{n} & 959 \\ \text { Gender, ratio male/female } & 0.58 \\ \text { Age, median (range) } & 54.7(16-86) \\ \text { Pack years, median (IQR) } & 40(25-60) \\ \text { Fagerström nicotine } & \text { Low }(18 \%) \text {; Moderate }(36 \%) \text {; } \\ \text { dependence test } & \text { High }(35 \%) \text {, Unknown }(11 \%) \\ \text { Motivation scale, points (IQR) } & 8(5-9) \\ \text { Self-confidence, points (IQR) } & 7(5-8)\end{array}$
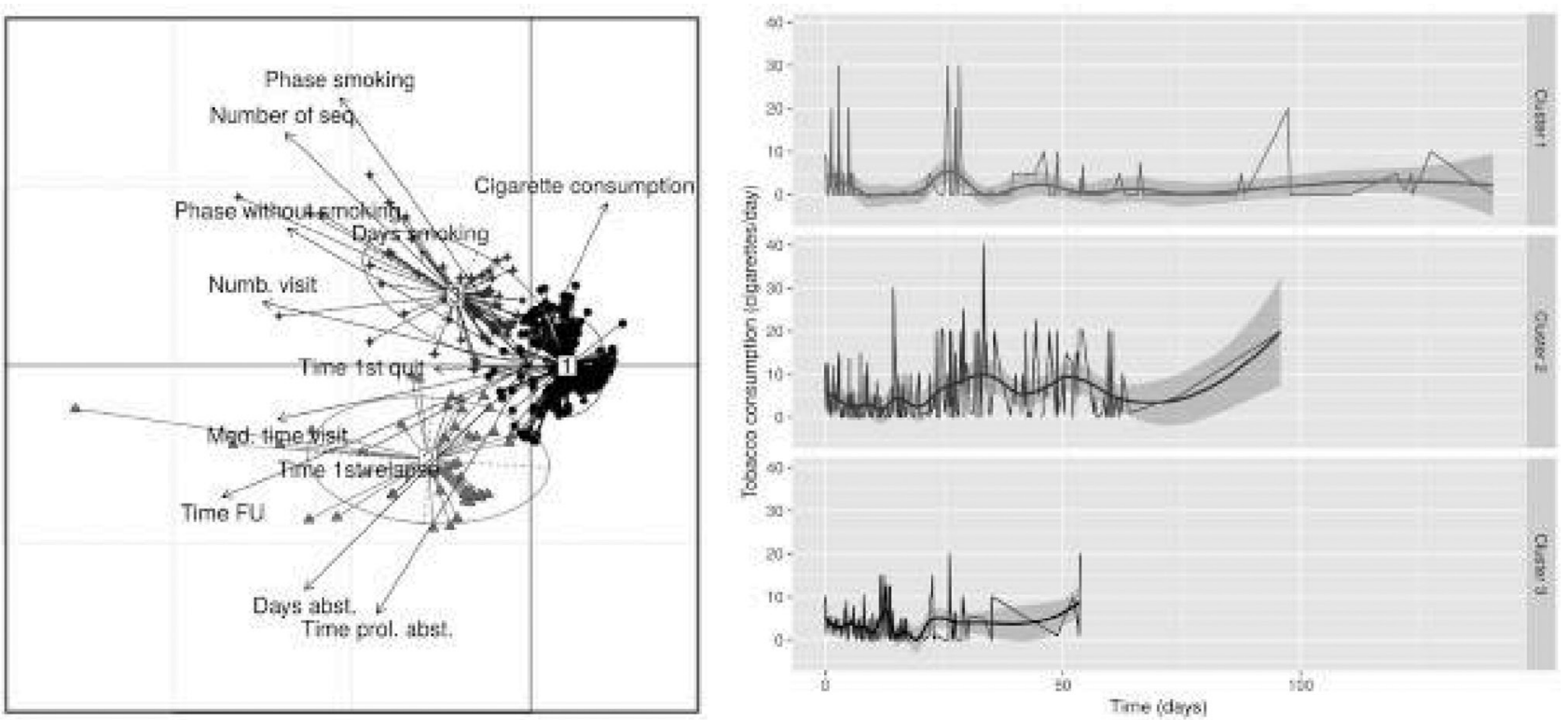

Fig. 1. Principal component analysis and patient clusters (for Abstract 151). 
intermediate follow-up period (cluster 2) and patients failing to quit within a short follow-up time (cluster 3 ). The clusters were independent from the dependence level (Fagerström) or the participant's exposure to tobacco (pack years).

Conclusion: We could identify a cluster of promising patients who gradually stopped smoking and remained abstinent for a prolonged period of time. Patients with a longer follow-up had a higher chance to quit. Daily measurements of tobacco consumption would allow the detection of finer behavioral patterns during smoking cessation.

Disclosure: Nothing to disclose

153

\section{Success Rates and Characteristics of Smoking Cessation Outpatient Clinic at the Cantonal Hospital in Winterthur}

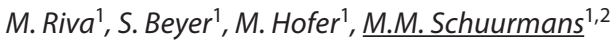 \\ 1Pulmonology, Cantonal Hospital Winterthur, Winterthur, \\ ${ }^{2}$ Pulmonology, University Hospital Zurich, Zurich, Switzerland
}

Introduction: Smoking cessation interventions are highly cost effective and considered standard of care for smokers requiring services from health care institutions. The smoking cessation outpatient clinic at the Cantonal Hospital Winterthur is run by a psychologist and a pulmonologist. Aim: Evaluation of counselling activities and cessation rates of patients attending the outpatient smoking cessation clinic in 2019.

Methods: Evaluation of smoking cessation activities performed and documented in a structured way. Cessation rates at one and three months. Biochemical verification of smoking status by exhaled CO measurement.

Results: In 2019 a total of 339 personal counselling sessions were performed. Additionally, 134 sessions were booked but were cancelled or the patient did not come for a scheduled counselling (no show). Most of the patients were referred by pulmonology (41\%) or by other departments of internal medicine (30\%), such as cardiology, oncology and gastroenterology. Abstinence rates at one and three months after initial consultation were 39\% (37/95) at one month and $42.5 \%$ at three months (31/73), respectively. Strong smoking reduction (few cigarettes) was observed in $6.3 \%$ of the patients at one month and $9.6 \%$ at three months. The most frequent medication support method was nicotine replacement $(62 / 135,46 \%)$, followed by Varenicline $(45 / 135,33 \%)$, and Bupropion $(11 / 135,8 \%)$. Some patients had more than one medication i.e. varenicline plus NRT nicotine inhaler. Some patients only received counselling (no medication: 17/135, 12.6\%).

There was a high rate of loss to follow up or missing data because the follow-up time has not been completed yet and is ongoing (30.1\% after one month and $46.3 \%$ after three months after the initial consultation). Final results will be reported when follow-up is completed (3 months end of March 2020).
Conclusions: In our population, we have a smoking-quit rate of $38.9 \%$ at one month and $42.5 \%$ at three months. Success rates for smoking cessation were approximately at the level of published rates although our population was more heterogenous in comparison to published studies.

Disclosure: No conflict of interest. The counselling project was supported by the lung league of the canton Zurich (Lunge Zürich). The sponsor had not influence on our counselling practice.

\section{9 \\ Association between Obstructive Syndrome and Mental Disorder - The Pneumolaus|Psycolaus Study}

\author{
B. Touilloux ${ }^{1}$, A. Lenoir ${ }^{1}$, J.-W. Fitting ${ }^{1}$, P. Vollenweider ${ }^{2}$, \\ M.-P.F. Strippoli ${ }^{3}$, M. Preisig ${ }^{3}$, L.P. Nicod ${ }^{1}$
}

${ }^{1}$ Division of Respiratory Medicine, ${ }^{2}$ Division of General Internal Medicine, Lausanne University Hospital (CHUV), ${ }^{3}$ Center for Research in Psychiatric Epidemiology and Psychopathology, Lausanne University Hospital (CHUV) and University of Lausanne, Lausanne, Switzerland

Mental health disorders figure among the many comorbidities of chronic obstructive pulmonary diseases. The purpose of this study is to evaluate the association of airway obstruction with depression and anxiety in the Swiss general population.

PneumoLaus is based on the CoLaus|PsyCoLaus cohort which extensively phenotyped a random sample of the Lausanne general population, Switzerland, including spirometry and a semi-structured interview on mental health. We defined reversible obstruction $(\mathrm{RO})$ as $\mathrm{FEV}_{1} / \mathrm{FVC}<\mathrm{LLN}$ or $\mathrm{FVC}<\mathrm{LLN}$ that became $\mathrm{FEV}_{1} /$ FVC $>$ LLN or FVC $>$ LLN after bronchodilation (BD). Chronic obstruction (CO) was defined as $\mathrm{FEV}_{1} / \mathrm{FVC}<\mathrm{LLN}$ after BD. Recent remitted depression or anxiety was defined as a diagnosis in the four years preceding the interview. We performed a cross-sectional analysis using logistic and linear regression models adjusted for social class, BMI, age, gender and smoking status.

In total, 2774 subjects performed spirometry and were assessed through the PsyCoLaus interview. We found evidence for associations of self-reported doctor-diagnosed asthma (OR 1.60, 95\%CI 1.06-2.39) and COPD, emphysema or chronic bronchitis (OR 2.61, 95\%CI 1.19-5.71) with recent remitted or current major depression (RCD). These associations were not found when spirometric criteria $\left(\mathrm{CO}, \mathrm{RO}, \mathrm{FEV}_{1}, \mathrm{FVC}\right.$ in \% predicted) were used instead of the self-reported diagnoses. There was some suggestion for associations between RO and RCD (OR 1.62, 95\%CI 0.87-3.01) and RO and recent remitted/current anxiety disorder (OR 1.95, 95\% CI 0.99-3.87).

Subjects with known diagnosis of obstructive respiratory disease more frequently suffer from depression and anxiety. However, this association was not confirmed when using objective spirometric values instead of self-reported diagnoses.

Disclosure: Nothing to disclose 


\section{1 \\ The association of FVC with quality of life - The PneumoLaus|PsyCoLaus study}

\section{B. Touilloux ${ }^{1}$, A. Lenoir ${ }^{1}$, J.-W. Fitting ${ }^{1}$, P. Vollenweider ${ }^{2}$, M.-P.F. Strippoli ${ }^{3}$, M. Preisig ${ }^{3}$, L.P. Nicod ${ }^{1}$}

${ }^{1}$ Division of Respiratory Medicine, ${ }^{2}$ Division of General Internal Medicine, Lausanne University Hospital (CHUV), ${ }^{3}$ Center for Research in Psychiatric Epidemiology and Psychopathology, Lausanne University Hospital (CHUV) and University of Lausanne, Lausanne, Switzerland

Low forced vital capacity (FVC) is associated with lung restriction and increased mortality. The purpose of this study was to evaluate the association of FVC, forced expiratory volume in one second $\left(\mathrm{FEV}_{1}\right)$ and spirometric restrictive pattern with quality of life (QoL).

PneumoLaus is a substudy of the population-based CoLaus|PsyCoLaus cohort study. A random sample of the general population of Lausanne, Switzerland underwent extensive phenotyping including spirometry and self-assessment questionnaires on QoL (Manchester Short Assessment of Quality of Life, MANSA). We defined probable restriction (PR) as FVC lower than the lower limit of normal (LLN) after bronchodilation (BD), using GLI 2012 reference values. In a cross-sectional analysis, we studied associations between QoL and $\mathrm{PR}, \mathrm{FEV}_{1}, \mathrm{FVC}$ through linear regression models adjusted for social class, BMI, age, gender, smoking status, exposure to fumes and second-hand smoking.

In total, 2054 subjects had available spirometry and completed the MANSA questionnaire. The mean MANSA score was lower in the PR group (4.70/7) than in the normal spirometry group (5.44/7; coefficient $b=-0.71, p<0.001)$. There was a positive association between MANSA score and the continuous variables $\mathrm{FEV}_{1}$ (\%predicted, coefficient $\mathrm{b}=0.33, \mathrm{p}=0.01$ ) and FVC (\%predicted, coefficient $b=0.43, p<0.001)$. Associations persisted when the model was adjusted for respiratory symptoms. When adjusted for $\mathrm{FEV}_{1}$, there was less evidence for an association of FVC (\%predicted) with MANSA score (coefficient $b=0.40, p=0.07$ ).

Lower FVC is associated with lower QoL compared to subjects with normal spirometric values. The mechanisms of this association could be related to environmental or personal factors and merit further investigation.

Disclosure: Nothing to disclose

\section{3 \\ Effect of High-Flow Oxygen on Exercise Performance in Copd. A Randomized Crossover Trial}

K. Bitos ${ }^{1}$, M. Furian ${ }^{1}$, L. Mayer ${ }^{1}$, S.R. Schneider ${ }^{1}$, S. Buenzli ${ }^{1}$, M. Mademilov', U. Sheraliev', A.K. Abdraeva'2, S. Aydaralieva' ${ }^{2}$, A. Myratbekova' ${ }^{2}$, T.M. Sooronbaev ${ }^{2}$, S. Ulrich ${ }^{1}$, K.E. Bloch ${ }^{1}$

${ }^{1}$ Department of Respiratory Medicine, University Hospital Zurich, Zurich, Switzerland, ${ }^{2}$ National Center for Cardiology and Internal Medicine, Bishkek, Kyrgyzstan

Background: High-flow oxygen therapy (HFOT) provides oxygen-enriched, humidified, heated air at high flow rates. It could be an alternative to low-flow oxygen therapy via nasal cannula (LFOT), which is commonly used by patients with chronic obstructive pulmonary disease (COPD) during endurance training in pulmonary rehabilitation. We hypothesized that HFOT improves exercise endurance in COPD patients compared to LFOT.

Methods: Patients with stable, moderate to severe COPD with resting pulse oximetry $\left(\mathrm{SpO}_{2}\right) \geq 92 \%$ performed two cycle constant work-rate exercise tests to exhaustion at $75 \%$ of their maximum work-rate on two different days, using first LFOT $(3 \mathrm{~L} / \mathrm{min})$, then $\mathrm{HFOT}\left(60 \mathrm{~L} / \mathrm{min}, \mathrm{FiO}_{2} 0.45\right)$, or vice versa, according to a randomized, crossover design. The primary outcome was the difference in endurance time between therapies. Secondary outcomes were respiratory rate, $\mathrm{SpO}_{2}$, heart rate, arterial blood gases and dyspnea.

Results: 79 patients, mean $\pm \mathrm{SD}$ aged 58 \pm 9years, $\mathrm{FEV}_{1}$ $63 \pm 9 \%$ predicted were randomized and included in the intentionto-treat analysis. Endurance time improved under HFOT versus LFOT by a mean of 85 s (95\%CI: 7 to 164), corresponding to an increase of $13 \%$ (95\%CI: 1 to 28). Under HFOT, patients had lower respiratory rate and higher $\mathrm{SpO}_{2}$ at isotime; at end-exercise under HFOT, $\mathrm{SpO}_{2}$ remained higher, $1.9 \%$ (1.5 to 2.3), and dyspnea sensation was lower, -0.8 points Borg CR10-scale (-1.2 to -0.3$)$, compared to LFOT.

Conclusions: HFOT improved endurance time in patients with stable, moderate to severe COPD by improving arterial oxygenation, respiratory rate and dyspnea compared to LFOT. Therefore, HFOT is promising for enhancing exercise performance in COPD. (ClinicalTrials.gov, NCT03955770)

Disclosure: Nothing to disclose 


\section{1 \\ Determinants for Treatment Change in a Swiss Copd Cohort Study}

L. Kleinsorge ${ }^{1}$, G. Lüthi-Corridori ${ }^{1}$, N. Abu Hussein ${ }^{2}$, P.O. Bridevaux ${ }^{3}$, C. Cadus' ${ }^{2}$ P.N. Chhajed' ${ }^{1}$, T. Geiser ${ }^{4}$, P. Grendelmeier ${ }^{1}$, L. Joos Zellweger ${ }^{5}$, M. Kohler ${ }^{6}$, S. Maier ${ }^{1}$, D. Miedinger ${ }^{1}$, M. Tamm², R. Turnheer ${ }^{7}$, A. Tschacher ${ }^{1}$, P. Urwyler ${ }^{1}$, J.D. Leuppi ${ }^{1,8}$

${ }^{1}$ University Clinic of Medicine, Cantonal Hospital Baselland, Liestal, ${ }^{2}$ University Hospital Basel, Basel, ${ }^{3}$ Hospital of Valais, University of Geneva, Geneva, ${ }^{4}$ University Hospital Bern, Bern, ${ }^{5}$ St. Clara Hospital, Basel, 6 University Hospital Zurich, Zürich, ${ }^{7}$ Cantonal Hospital Muensterlingen, Münsterlingen, ${ }^{8}$ University of Basel, Basel, Switzerland

Introduction: Chronic obstructive pulmonary disease (COPD) is a widely spread chronic disease. It is estimated that 65 million patients worldwide and 400'000 patients in Switzerland suffer from the disease. The Swiss COPD Cohort Study (SCCS) was established in 2006 to asses COPD management in a primary care setting. To date little is known about factors associated with changes in treatment among patients with COPD. We thus evaluated predictors for treatment adjustments to understand what drives general practitioners to change therapy of COPD patients.

Method: The SCCS is an ongoing questionnaire-based Study with the objective to evaluate COPD management in primary care. General practitioners recruited patients with a spirometrically diagnosed COPD. Every 6 months information about symptoms (by CAT- and mMRC-score), exacerbations, treatment and comorbidities were assessed and spirometry was performed. Data of patients included from 2015-2019 were used for this study and were screened for therapy change. A logistic mixed model was performed to detect predictors.

Results: 195 patients with 690 visits were included in the study. The mean age was 68.1 (37-90) years, $35.4 \%$ were female, the mean FEV1 was $60.9( \pm 18.13)$ and most patients had COPD GOLD A or $\mathrm{B}(81.5 \%) .69 \%$ of the visits were without therapy changes. In $31 \%$, a treatment adjustment was detected, whereof $34 \%$ presented a step-up in therapy, $32 \%$ a step-down and $34 \%$ a step-up and stepdown at the same time.

Exacerbation, GOLD stage C, D and severe airway obstruction GOLD 3-4 were associated with treatment changes. If comorbidities were present, therapy changes were less likely to be made.

Conclusion: This analysis demonstrates that in most visits there were no therapy changes. If adjustments were made, they were linked to exacerbation, hospitalization and severity of airflow limitation. Surprisingly comorbidities showed a negative association with therapy changes.

Disclosure: Nothing to disclose

\section{1 \\ Oral Corticosteroid Prescription in Asthma: Swiss Situation}

C. Leuenberger ${ }^{1}$, J.D. Leuppi ${ }^{2}$, T. Rothe ${ }^{3}$, A. Jochmann ${ }^{4}$,

C. Clarenbach ${ }^{5}$, C. Steurer-Stey ${ }^{6}$, F. Charbonnier ${ }^{7}$, H.-W. Duchna ${ }^{8}$,

P.-O. Bridevaux ${ }^{9}$, V. Chicherov ${ }^{10}$, M. Korobeynikova ${ }^{10}$, S. Wetzel ${ }^{10}$,

G. Pendl ${ }^{1}$

${ }^{1}$ AstraZeneca Switzerland AG, Baar, ${ }^{2}$ Medizinische

Universitätsklinik Kantonsspital Baselland,

Liestal, ${ }^{3}$ Kantonsspital AarauGraubünden, Chur,

${ }^{4}$ Universitätskinderspital beider Basel, Basel, ${ }^{5}$ Universitätsspital

Zürich, ${ }^{6}$ Epidemiology, Biostatistics and Prevention Institute,

Universität Zürich and MediX Gruppenpraxis, Zürich, ${ }^{7}$ Hôpitaux

Universitaires Genève, Geneva, ${ }^{8}$ Hochgebirgsklinik Davos,

Davos, ${ }^{9}$ Hôpital du Valais, Sion, Sion, ${ }^{10}$ IQVIA Solutions $\mathrm{GmbH}$,

Rotkreuz, Switzerland

Introduction: Severe refractory asthma is characterized by a higher risk of asthma-related symptoms, comorbidities, and exacerbations. Airway eosinophilia is present in most asthmatic patients, and elevated levels of blood and sputum eosinophils are
Fig. 1. Fulfilled OCS strength (prednisone equivalent in $\mathrm{mg}$ ) and pill volume for Asthma (period Nov. '18 - O (for Abstract 201)

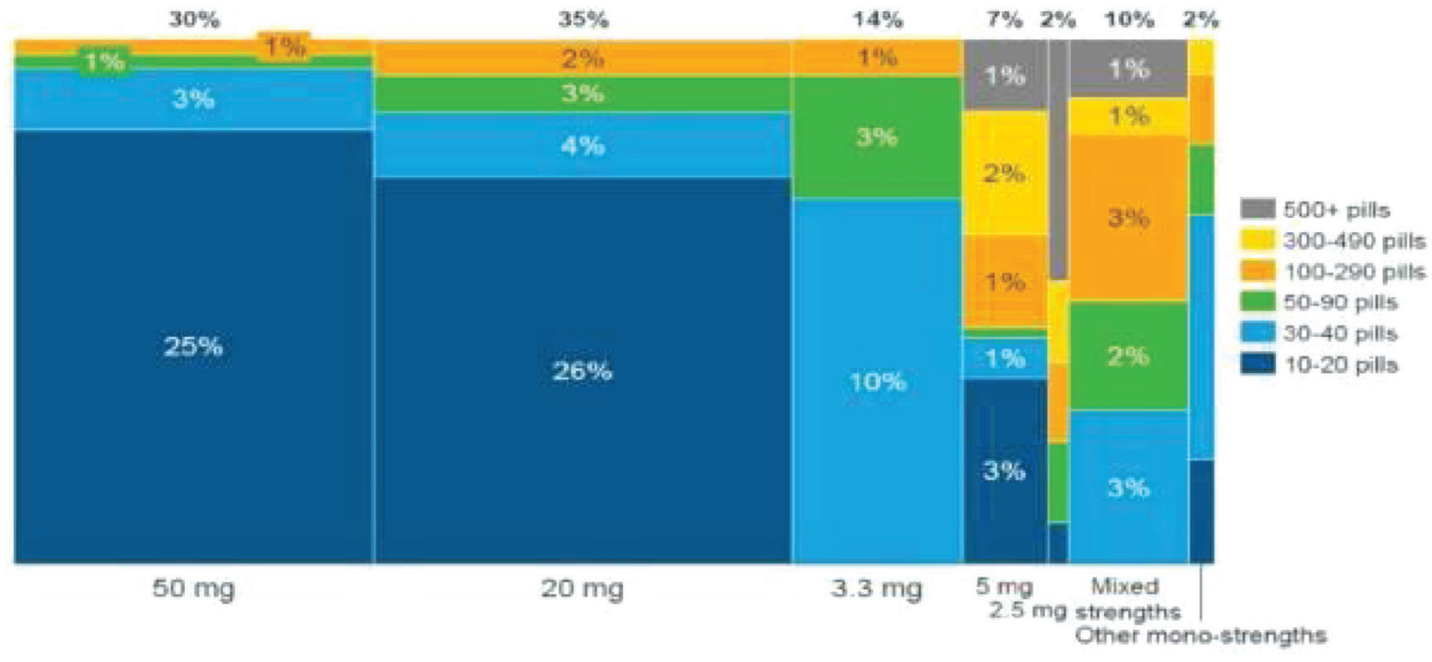

Respiration 2020;99:700-737 
associated with worse asthma control. Since patients with eosinophilic asthma respond to oral corticosteroids (OCS) we investigated the prescription pattern for Switzerland. 1-2g prednisone equivalent per year is associated with long-term side effects with a heavy burden for patients and incremental health-care costs.

Methods: This is a retrospective study of anonymized patient level prescription data from Swiss pharmacies (IQVIA Swiss pharmacy $\mathrm{LRx}^{\oplus}$ database) during the period of November 2016 to October 2019 (3 years). Asthma patients were classified based on patient gender, age and historic medication by a predictive model trained on prescription data with diagnoses (IQVIA Disease Analyzer $^{\circledast}$ ) from IQVIA Germany.

Results: To select for asthma patients on regular treatment, 8.145 patients were identified who had at least one asthma specific prescription in each year in the inclusion period. From these $10-11 \%$ had an OCS prescription in each year, $21 \%$ at least once between Nov. '16 and Oct. '19. In 779 patients receiving OCS in the last year, $8 \%$ received OCS likely due to comorbidities other than asthma, 79\% were classified as using OCS in the acute setting and $14 \%$ chronically based on pill possession rate (>100 pills/year). Whereas for chronic users OCS median yearly cumulative dose was $2.000 \mathrm{mg}$ prednisone equivalent, the median in the acute setting was $400 \mathrm{mg}$ respectively. Most notably $23 \%$ of all OCS patients received more than $1.000 \mathrm{mg}$ prednisone equivalent OCS per year.

Conclusion: In Switzerland OCS prescriptions in asthma patients is mainly for acute use. However, every $4^{\text {th }}$ patient receiving OCS fulfills prescriptions with a cumulative dose of $>1 \mathrm{~g}$ per year and should be evaluated for biologic therapy.

Disclosure: Jörg D. Leuppi: grant research support from AstraZeneca, Boehringer Ingelheim, GSK, Mundipharma, Novartis, Sanofi and advisory and lecture fees from AstraZeneca, Boehringer Ingelheim, GSK, Novartis, Sanofi Thomas Rothe: advisory fees from Astra Zeneca, GSK, TEVA, Novartis and lecture fees from Astra Zeneca, GSK, Novartis; Christian Clarenbach: advisory fees from Roche, Novartis, Boehringer, GSK, Astra Zeneca, Sanofi, Vifor and Mundipharma; Claudia Steurer-Stey: advisory boards and speaker fees from Astra Zeneca, GSK, Novartis, Boehringer Ingelheim; Vitaly Chicherov, Maria Korobeynikova and Stefan Wetzel are employees of IQVIA, which conducted the presented work under contract for AstraZeneca; Caroline Leuenberger and Gunther Pendl are employees of Astra Zeneca;

\section{5 \\ Bronchial Thermoplasty May Increase Steroid Sensitivity by Upregulating the Expression of the Glucocorticoid Receptor}

\author{
L. Zhou' ${ }^{1}$, L. Fang ${ }^{2}$, D. Stolz ${ }^{2}$, M. Tamm², M. Roth ${ }^{2}$ \\ ${ }^{1}$ Department of Medicine, University of Basel, ${ }^{2}$ University \\ Hospital of Basel, Basel, Switzerland
}

Background: Bronchial thermoplasty is applied to patients with severe asthma. Besides reducing airway wall remodeling and increasing quality of life, it was reported that the use of steroid could be reduced. We reported earlier that glucocorticoid receptor (GR) regulating heat shock proteins were affected by bronchial thermoplasty.

Aim: In vitro assessment regarding the effect of heat on the expression of the GR by airway smooth muscle cells (ASMC) and epithelial cells (EC).

Methods: Immortalized human bronchial epithelium (BEAS$2 B)$, human primary EC $(n=14)$ and ASMC $(n=27)$ were treated with increasing temperatures $\left(37,45,55,65^{\circ} \mathrm{C}\right)$ for 10 seconds. The cells were incubated for $24 \mathrm{~h}$ and $48 \mathrm{~h}$, in the presence and absence of steroids $\left(10^{-8} \mathrm{M}\right)$. GR expression was determined by $\mathrm{qPCR}$, Western-blotting and immunohistochemistry.

Results: ASMC were significantly more sensitive to heat than EC. ASMC and BEAS-2B cells showed signs of apoptosis at temperatures above $50^{\circ} \mathrm{C}$, while primary EC showed no sign of cell death. Exposure to $65^{\circ} \mathrm{C}$ reduced the expression of the GR in ASMC without any significant difference between controls, asthma, and COPD patients at $24 \mathrm{~h}$ or $48 \mathrm{~h}$. In contrast, increasing heat significantly upregulated the expression of the GR in EC, but not in BEAS-2B cells. The cell type specific response of the GR to heat was confirmed on the level of transcription, and protein expression.

Conclusion: Bronchial thermoplasty or heat application modifies the expression of the GR in a cell type specific manner. This may explain the reduced requirement of steroids for asthma therapy after bronchial thermoplasty.

Disclosure: Nothing to disclose

\section{5 \\ Exogenous HSP60 Stimulates Airway Smooth Muscle Cell Remodeling via Mapk p38 Pathway

\author{
J.Li' ${ }^{1}$, L. Fang ${ }^{2}$, D. Stolz ${ }^{2}$, M. Tamm ${ }^{2}$, M. Roth ${ }^{2}$ \\ Switzerland
} \\ ${ }^{1}$ Department of Biomedicine, ${ }^{2}$ University of Basel, Basel,}

Background: Asthma is characterized by airway inflammation and remodeling. We reported earlier that heat shock protein-60 (HSP60) was secreted by bronchial epithelial cells of asthma patients. HSP60 activated mitogen-activated protein kinases p38, thereby controlling tissue remodeling and the action of CCAAT/ enhancer-binding proteins $(\mathrm{C} / \mathrm{EBP}) \alpha / \beta$.

Objective: The effect of exogenous human recombinant HSP60 (eHSP60) on the activation of p38 and C/EBPs, as well as on remodeling of airway smooth muscle cells (ASMC).

Methods: ASMC were obtained from healthy controls and asthma patients. ASMC were treated with eHSP60 $(10 \mu \mathrm{M})$ and/or p38 inhibitors (SB2053580, $10 \mu \mathrm{M})$. The cellular proteins were collected and the expression of total p38, phosphorylated p38, C/ $\mathrm{EBP}-\alpha / \beta$, fibronectin, $\alpha$-smooth muscle actin (SMA) and collagenI were measured by Western-blotting.

Results: Treatment with eHSP60 significantly increased the phosphorylation of $\mathrm{p} 38$ and the expression of $\mathrm{C} / \mathrm{EBP}-\alpha / \beta$, fibronectin, SMA and collagen-I. Inhibition of p38 decreased eHSP60induced phosphorylation of $\mathrm{p} 38$, and expression of $\mathrm{C} / \mathrm{EBP}-\alpha / \beta$, fibronectin, SMA and collagen-I. In healthy control ASMC, 
eHSP60 did neither increase phosphorylation of p38, nor expression of $\mathrm{C} / \mathrm{EBP}-\alpha / \beta$, fibronectin, SMA and collagen-I.

Conclusion: This is the first report that ASMC of asthma patients respond in a disease specific manner to HSP60 by activation of p38. This suggests HSP60 to be a novel target for therapy and diagnosis.

Disclosure: Nothing to disclose

\section{6 \\ Antecedent Hospitalization History of Patients Newly Diagnosed with COPD}

\author{
F. Baty ${ }^{1}$, M. Hagmann ${ }^{2}$, M. Brutsche 1 \\ ${ }^{1}$ Lung Center, Cantonal Hospital St. Gallen, St. Gallen, \\ ${ }^{2}$ University of Basel, Basel, Switzerland
}

Introduction: A number of risk factors can contribute to the development of chronic obstructive pulmonary disease (COPD). Many patients with COPD remain undiagnosed and it is important to identify factors allowing early diagnosis of COPD. Hospitalization occurring prior the initial assessment of COPD could reveal key factors associated with a future onset of COPD. Our aim was to investigate predisposing factors of COPD by looking at the antecedent hospitalization history of patients newly diagnosed with COPD.

Material and methods: A nation-wide hospitalization database provided by the Swiss Federal Office for Statistics including information about all hospitalizations in Switzerland from 2002 until 2017 was analyzed. Data included a list of diagnoses / comorbidities (ICD-10 codes), the date of admission, gender, age, length of stay and in-hospital mortality. Anonymous unique identifiers of patients with COPD were used to track their hospitalization history and investigate the evolution of comorbidities leading to a COPD diagnosis. Between-class principal component analysis was used to explore the time evolution of pre-COPD comorbidities.

Results: In total, $641^{\prime} 556$ hospitalizations included a COPD diagnosis (J44*) between 2002 and 2017. Tracking back individual hospitalizations in patient prior initial COPD assessment revealed a series of both common and in the context of COPD underrecognized comorbidities (Figure 1). Early diagnoses of nicotine- (F172), alcohol-dependence (F102) and obesity (E669) were followed by diagnoses of atherosclerotic heart diseases (I25) and essential hypertension (I10). Comorbidities occurring at a later stage close to the initial COPD diagnosis included atrial fibrillation (I48), diseases of the genitourinary system (N390, N189), pneumonia (J189) and finally hypertensive heart diseases (I1190) and type 2 diabetes (E1190). Hospitalizations tended to be more frequent over time and with a longer length of stay.
Fig. 1. Comorbidities in the antecedent hospitalization history of patients with COPD (for Abstract 256).

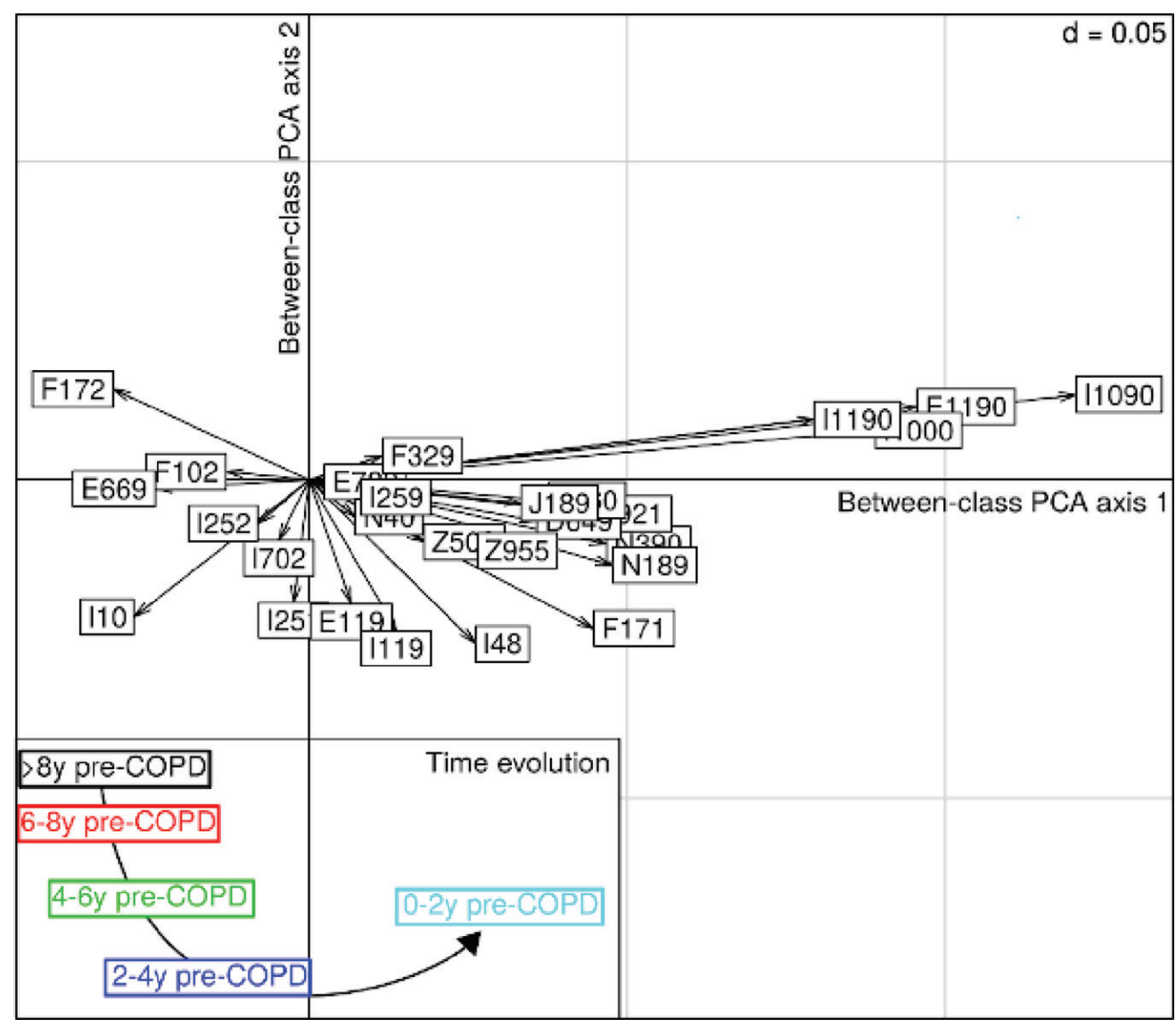


Conclusion: The longitudinal assessment of comorbidities recorded in the hospitalization history of patients at risk for COPD provides early indicators of future onset of COPD.

Disclosure: Nothing to disclose

\section{3 \\ Electrocardiographic Changes and Cardiac Ischemia in Lowlanders with COPD Travelling to $3100 \mathrm{~m}$}

\author{
A.F. Carta ${ }^{1}$, M. Furian ${ }^{1}$, M. Lichtblau ${ }^{1}$, K. Bitos ${ }^{1}$, S.R. Schneider ${ }^{1}$, \\ M. Mademilov' ${ }^{2}$ U. Sheraliev², N.H. Marazhapov², \\ T.M. Sooronbaev ${ }^{2}$, K.E. Bloch' ${ }^{1}$ S. Ulrich ${ }^{1}$ \\ ${ }^{1}$ Pneumologie, Universitätsspital Zürich, Zürich, Switzerland, \\ ${ }^{2}$ Department of Respiratory Medicine, National Center for \\ Cardiology and Internal Medicine, Bishkek, Kyrgyzstan
}

Background: The safety of altitude sojourns in patients with chronic obstructive pulmonary disease (COPD) is insufficiently known. We investigated ECG-changes at rest and during exercise in COPD patients travelling to $3100 \mathrm{~m}$ with focus on ischemia.

Methods: Kyrgyz lowlanders (residence $<800 \mathrm{~m}$ ) with COPD (FEV1 $40-80 \%$ predicted; $\mathrm{SpO}_{2} \geq 92 \% ; \mathrm{PaCO}_{2}<6 \mathrm{kPa}$ at $760 \mathrm{~m}$, 18 to 75 years, without symptoms or ECG signs of active coronary heart disease) underwent 12-lead ECG recordings at rest and during cycle incremental exercise tests (IET) at $760 \mathrm{~m}$ and $3100 \mathrm{~m}$. ECGs were scored for ischemia ( $\geq 1 \mathrm{~mm}$ horizontal or downsloping

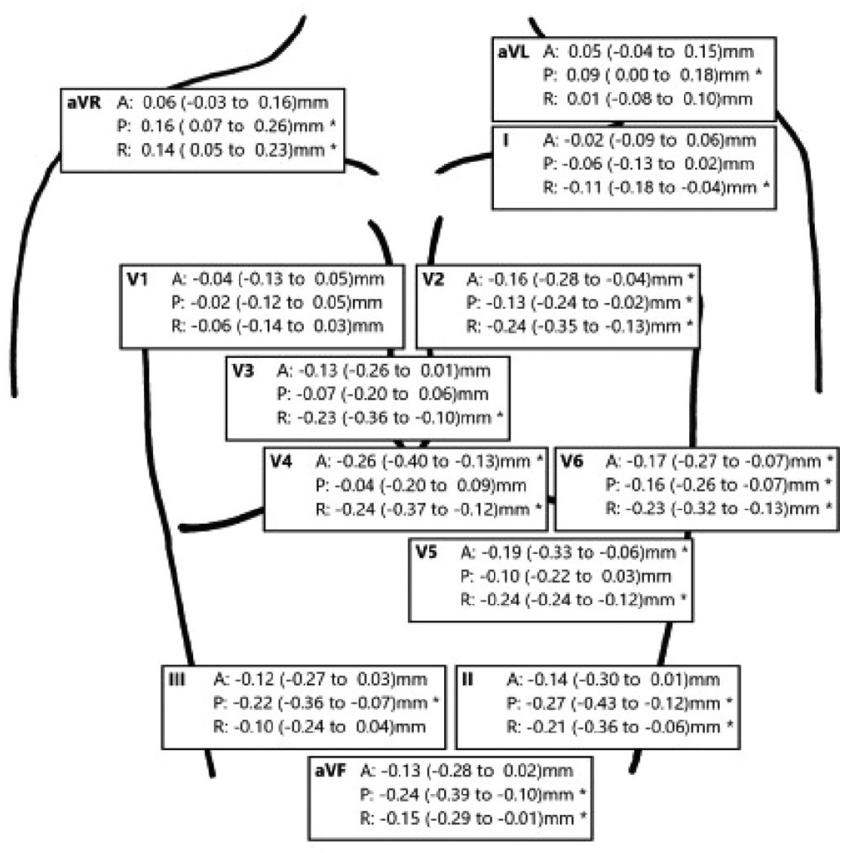

Fig. 1. ST segment changes (Mean and 95\% confidence interval) A: Altitude (760m to $3100 \mathrm{~m})$, P: rest to Peak Exercise, R: rest to Recovery (for Abstract 263).
ST segment depression) and mean ST-changes averaged over 10 seconds at rest, peak exercise and 2-minute recovery.

Results: 80 COPD patients ( $51 \%$ female, $56.2 \pm 9.6$ years, BMI $27.0 \pm 4.5$, SpO2 93.9 $\pm 1.8 \%$, FEV1 $63 \pm 10 \%$ pred.) were included. 64 patients performed IET at $760 \mathrm{~m}, 53$ at $3100 \mathrm{~m}$. At $3100 \mathrm{~m} 2(3.7 \%)$ patients revealed ischemia on ECG vs 0 at $760 \mathrm{~m}(\mathrm{p}=0.20)$. Figure 1 shows mean ST changes.

Conclusion: This first study investigating ischemia by ECG at rest and during exercise in COPD patients travelling to altitude did not reveal an increased incidence of ischemia. However, statistically significant ST changes well below the threshold of clinical relevance were detected in multiple leads.

Disclosure: Nothing to disclose

\section{9 \\ Frequency and Significance of Pathologic Pulmonary Findings in Postmortal Diagnostics}

\section{A. Schmid, S. Berezowska, M. Trippel, A. Blank, Y. Banz, A. Lugli, R. Langer}

Institute of Pathology, University of Bern, Bern, Switzerland

Clinical diagnostics of unclear pulmonary findings is challenging and the discrepancy between clinical and postmortal pathologic findings is a well-known phenomenon. We performed a comprehensive analysis of postmortal pulmonary findings in adults in correlation with clinical diagnoses and questions.

As standard approach, the lungs were fixed after formalin instillation for at least 24 hours followed by slicing large tissue sections and a subsequent standardized histological work-up including macroscopically abnormal areas. The consecutive cases were prospectively collected during a period of two years and analyzed retrospectively.

In total, 189 patients were included: 66 cases from the university hospital including 32 from the intensive care unit (ICU), and 123 from peripheral hospitals. 130 patients had significant primary pathologic findings in the lung (congestion due to cardiac failure excluded): 13 cases showed diffuse alveolar damage (DAD), 29 cases inflammatory conditions, 46 cases emphysema or degenerative changes, 27 cases primary or secondary tumors, and 15 cases other pathologies. Pulmonary disease was the primary cause of death in 26 cases. In 14 cases the cause of death was combined cardio-pulmonary failure. A complete correlation between clinical and pathological diagnoses was present in 75 cases; in 57 cases a specific finding was clinically suspected and confirmed by autopsy; unclear clinical findings specified by autopsy were present in 16 cases. Discrepant findings were seen in 31 cases, among them cases with previous suspicion for a specific finding, which was not confirmed at autopsy in 9 cases and completely new findings not diagnosed before death in 32 cases. The latter categories included major diagnostic discrepancies according to the Goldman criteria in 24 cases. DAD was more frequent in patients from the ICU compared to other clinics and peripheral hospitals $(\mathrm{p}=0.044)$. Besides, there was no other significant differences between the different clinics, in particular regarding the frequencies of diagnoses and discrepant findings.
SSC/SSCS SSP/SSTS

Joint Annual Meeting 2020 
Pulmonary pathologies present a frequent finding in postmortal diagnostics. Careful investigation is beneficial for the quality of postmortal diagnostics and a reliable clinico-pathologic correlation, which should not only focus on discrepant findings but also value confirmatory results or solutions to clinically unclear of only suspected conditions.

Disclosure: Nothing to disclose

\section{6 \\ Cell Type and Disease Specific Expression of the GPR68 in the Human Lung}

\author{
S. Blumer ${ }^{1}$, L. Fang ${ }^{1}$, M. Tamm ${ }^{2}$, D. Stolz ${ }^{2}$, M. Roth ${ }^{2}$ \\ ${ }^{1}$ Department Biomedicine, ${ }^{2}$ Universitätsspital Basel, \\ Basel, Switzerland
}

Introduction: The GPR68 is a seven transmembrane G-protein coupled receptor sensing protons and thus responding to changes of extracellular acidity. Tissue acidification occurs under hypoxic stress, metabolic alteration or during tissue remodeling. It is a defining hallmark in chronic inflammatory lung disease, but the role of GPR68 in Asthma and COPD is not well studied.

Methods: Tissues and primary human lung cells were obtained by endo-bronchial biopsies of eight controls, ten asthma patients and five patients with COPD. GPR68 expression was determined by three independent methods: real time-quantitative PCR, immunoblot and immunohistology.

Results: GPR68 was expressed by human primary epithelial cells, airway smooth muscle cells and fibroblasts. Cell type specific differences of GPR68 expression were observed by comparing the same diagnosis between different cell types. In control tissues and cells, the GPR68 expression was similar in epithelial and airway smooth muscle cells, while it was low in fibroblasts. In asthma and COPD derived tissues and cells, the GPR68 expression was highest in epithelial cells, lower in airway smooth muscle cells and lowest in fibroblasts. Disease specific differences of GPR68 expression were observed by comparing the same cell type between different diagnosis. In epithelial cells, the GPR68 expression was similar in all diagnostic groups, among which COPD patients showed the highest variability. In airway smooth muscle cells and fibroblasts, the GPR68 expression was highest in control cells and lower in asthmatic and COPD cells.

Conclusion: GPR68 expression was cell type and disease specific. This might indicate its contribution to chronic inflammatory lung diseases. It has to be determined if the GPR68 expression patterns correlate with its function.

Disclosure: Nothing to disclose

\section{Interstitial and Occupational Respiratory Disorders}

\section{Acute Lupus Pneumonitis as the Initial Presentation of Systematic Lupus Erythematosus. A Case Report}

C. Cantero, R. Vongthilath, J. Plojoux

Geneva University Hospitals, Geneva, Switzerland

Background: Acute lupus pneumonitis (ALP) is a rare complication of systemic lupus erythematosus (SLE). Due to its resemblance with pneumonia, its diagnosis is often missed and delayed. The objective of this clinical case report is to highlight this unusual injury to avoid incorrect diagnosis.

Case presentation: A 36-year-old South American female treated for a Hashimoto's disease, with irrelevant family history, was referred to the emergency department due to intermittent fever, fatigue, weight loss, mild dyspnea, dry cough with infrequent clear sputum, hair loss, myalgia and oral ulcers. Laboratory findings showed pancytopenia with lymphopenia. C-reactive protein was normal but procalcitonin and erythrocyte sedimentation rate

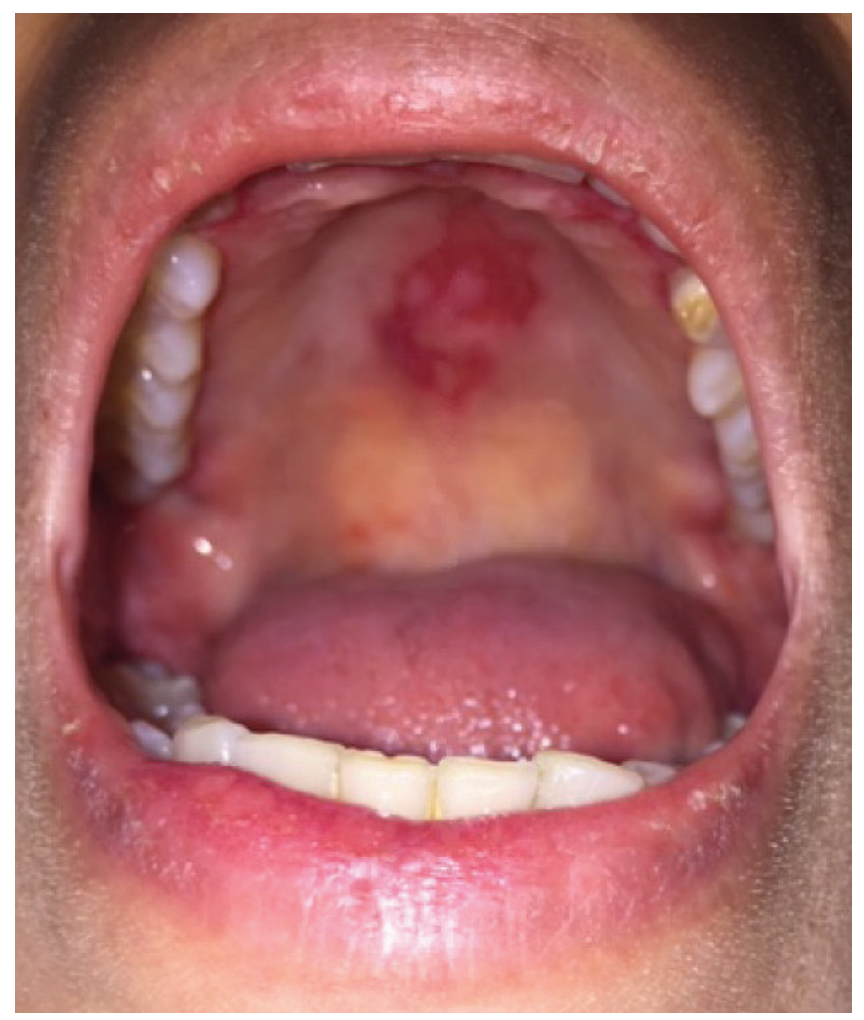

Fig. 1. Oral ulcers (for Abstract 5). 
Fig. 1. High-resolution computed tomography of the lungs showing alveolar condensation with air bronchograms (for Abstract 5).
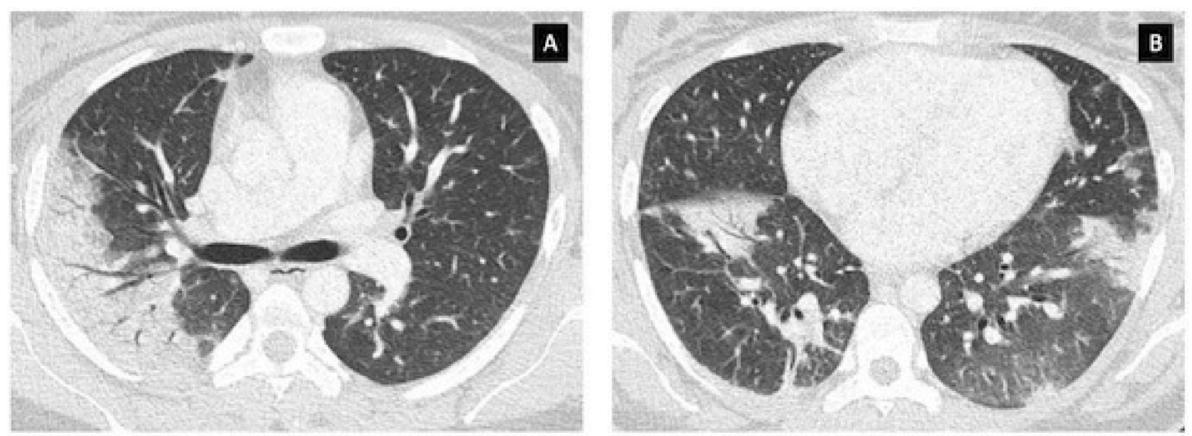

were elevated. Chest x-ray showed multiple and bilateral pulmonary opacities and high-resolution computed tomography of the lungs revealed numerous areas of alveolar condensation with air bronchograms. Patient was diagnosed with community-acquired multi-lobar pneumonia. Despite empirical antibiotic coverage and negative microbiological work-up, patient remained febrile without improvement of symptoms. Autoimmune markers were measured revealing highly elevated anti-nuclear antibodies, antidsDNA antibodies and positive antiphospholipid antibodies. Patient was diagnosed with SLE presenting with ALP, hematologic and mucocutaneous manifestations. Before initiating immunosuppressive therapy, bronchoscopy with bronchoalveolar lavage was performed and ruled out opportunistic infections and diffuse alveolar hemorrhage. Pulmonary function test revealed severe restrictive ventilatory defect.

Table 1. Autoimmune markers (for Abstract 5)

\begin{tabular}{|c|c|c|}
\hline & $\begin{array}{l}\text { Patient } \\
\text { results }\end{array}$ & $\begin{array}{l}\text { Normal } \\
\text { range }\end{array}$ \\
\hline anti-nuclear antibodies (titer) & $1: 5^{\prime} 000$ & $<1: 80$ \\
\hline anti-dsDNA antibodies (Ul/ml) & 1’044 & $<36$ \\
\hline $\mathrm{C} 3(\mathrm{~g} / \mathrm{l})$ & 0.28 & $0.66-1.35$ \\
\hline $\mathrm{C} 4(\mathrm{~g} / \mathrm{l})$ & 0.03 & $0.08-0.34$ \\
\hline anti-cardiolipin antibodies (U/ml) & 58.4 & $0-14$ \\
\hline anti- $\beta 2 \mathrm{GP} 1$ antibodies $(\mathrm{U} / \mathrm{ml})$ & $>100$ & $0-17$ \\
\hline Lupus anticoagulant (quantitative) & positive & negative \\
\hline
\end{tabular}

Table 2. Bronchoalveolar lavage (for Abstract 5)

\begin{tabular}{lcc}
\hline & $\begin{array}{l}\text { Patient } \\
\text { results }\end{array}$ & $\begin{array}{l}\text { Normal } \\
\text { range }\end{array}$ \\
\hline Total cell counts with differential (M/l) & 600 & $30-350$ \\
Cell viability (\%) & 99 & - \\
Macrophage (\%) & 73 & $55-97$ \\
Lymphocyte (\%) & 21 & $2-34$ \\
Neutrophil (\%) & 6 & $0-3$ \\
Hemosiderin (\%) & 0 & $0-25$ \\
\hline
\end{tabular}

Respiration 2020;99:700-737 DOI: $10.1159 / 000508318$
Treatment and outcome: Clinical, biological, radiological and functional improvement was noticed with the administration of steroids, hydroxychloroquine and immunoglobulin. Alveolar condensation with air bronchograms resolved and forced expiratory volume in 1 second went from $38 \%$ to $72 \%$ of the predicted after 6 weeks of treatment.

Discussion: ALP is described as being a rare manifestation of SLE with poor prognosis. However, its prevalence and mortality rate are rarely estimated, partly due to unreported or misdiagnosed cases. ALP should be suspected in patients with known SLE presenting acute constitutional and respiratory symptoms. In patients without known SLE, ALP must be suspected in cases of non-response to conventional treatment for pneumonia and when extrapulmonary characteristics of SLE are noted.

Disclosure: Nothing to disclose. Patient consent to publication of images and informations about her medical case. A consent form was signed by the patient and available for proof.

\section{3 \\ Role of Extracellular Vesicles in Chemotherapy- Induced Lung Metastasis}

\author{
N. Mansouri ${ }^{1,2}$, I. Keklikoglou ${ }^{3}$, S. Nassiri ${ }^{3}$, A. Guichard ${ }^{3}$, B. Torchia ${ }^{3}$, \\ M. De Palma ${ }^{3}$
}

${ }^{1}$ University of Lausanne (UNIL), Centre Hospitalier Universitaire Vaudois (CHUV), ${ }^{2}$ Swiss Institute for Experimental Cancer Research (ISREC), School of Life Sciences, École Polytechnique Fédérale de Lausanne (EPFL), ${ }^{3}$ Swiss Institute for Experimental Cancer Research (ISREC), School of Life Sciences, École Polytechnique Fédérale de Lausanne, EPFL, Lausanne, Switzerland

Introduction: Complete primary tumor shrinkage following neoadjuvant chemotherapy is frequently associated with a good prognosis. However, chemoresistant tumors face a high risk of future recurrence and metastatic disease. Recent work has suggested that certain forms of chemotherapy induce systemic host responses that may facilitate metastasis from chemoresistant mammary tumors. We have recently shown that chemotherapy induces mammary cancer cells to release extracellular vesicles (EVs) with altered protein composition and enhanced 
pro-metastatic capacity. Identifying and blocking the mechanisms that promote chemotherapy-induced lung metastasis may improve treatment efficacy in breast cancer patients receiving neoadjuvant chemotherapy.

Objectives: We profiled the proteomes and studied the effects of chemotherapy-induced, tumor-derived EVs on the mouse pulmonary niche.

Methods: The proteomes of EVs isolated from paclitaxel (chemo-EVs) or vehicle-treated (control-EVs) 4T1 mouse mammary tumor cells were analysed using mass spectrometry. Tumor-free mice were injected with 3 doses of chemo-EVs or control-EVs and the lung tissue was analysed by single-cell RNA sequencing and flow cytometry. Canonical marker genes were used for cell type annotation and differential gene expression was analysed in each cell cluster.

Results: Mass spectrometry analysis revealed the abundance of multiple pro-metastatic proteins in chemo-EVs. Single-cell RNA sequencing revealed differential gene expression in chemo-EV versus control-EV-treated mice, some of which may be associated with progression of metastasis.

Conclusions: We comprehensively profiled the proteome of EVs released from chemotherapy-treated cancer cells, and the transcriptome of the lung tissue exposed to chemotherapy at the single cell level. This work may help to identify molecular pathways potentially relevant to chemotherapy- and EV-induced metastasis.

Disclosure: Nothing to disclose

\section{3 \\ Effect of Nocturnal Oxygen Therapy on Next-Day Exercise Performance of COPD Patients at 2048m. Data from a RCT}

S. Gutweniger T.D. Latshang, S.S. Aeschbacher, F. Huber, D. Flueck, M. Lichtblau, S. Ulrich, E.D. Hasler, P.M. Scheiwiller, S. Ulrich, K.E. Bloch, M. Furian

Department of Respiratory Medicine, University Hospital Zurich, Zurich, Switzerland

Purpose: During altitude travel, exercise performance in patients with chronic obstructive pulmonary disease (COPD) is strongly reduced (Furian 2018). We evaluated whether nocturnal oxygen therapy (NOT) during a stay at $2048 \mathrm{~m}$ improves next-day exercise performance in lowlanders with COPD.

Methods: 32 lowlanders with COPD, mean $\pm \mathrm{SE} \mathrm{FEV}_{1}$ $54 \pm 9 \%$ predicted, living $<800 \mathrm{~m}$, stayed for 2 days at $2048 \mathrm{~m}$ twice, once with NOT, once with placebo according to a randomized, crossover trial with a 2 -week washout period at $<800 \mathrm{~m}$ in-between. Constant-load bicycle exercise to exhaustion at $60 \%$ of maximal workload was performed at $490 \mathrm{~m}$ and on day 2 of sojourns at $2048 \mathrm{~m}$ to measure endurance time as the primary outcome. Additional outcomes were altitude-related adverse effects (ARAHE) requiring treatment or descent. www.ClinicalTrials.gov NCT02150590.
Results: Mean \pm SE endurance time at $490 \mathrm{~m}$ was $602 \pm 64 \mathrm{~s}$, at $2048 \mathrm{~m}$ with placebo $326 \pm 62 \mathrm{~s}$ and with NOT $296 \pm 60$ s, mean difference (95\%CI) NOT-placebo -30s (-149 to 89), P=0.619. End-exercise values in pulse oximetry, cerebral tissue oxygenation and minute ventilation at $490 \mathrm{~m}$ were: $\mathrm{SpO}_{2}=92 \pm 1 \%$, CTO $=65 \pm 1 \%$, $\mathrm{V}^{\prime} \mathrm{E}=37.7 \pm 2.0 \mathrm{l} / \mathrm{min}$; during sojourns at $2048 \mathrm{~m}$ with placebo: $\mathrm{SpO}_{2}=85 \pm 1 \%, \mathrm{CTO}=61 \pm 1 \%, \mathrm{~V}^{\prime} \mathrm{E}=40.6 \pm 2.0 \mathrm{l} / \mathrm{min}$ and during sojourns with $\mathrm{NOT}$ : $\mathrm{SpO}_{2}=84 \pm 1 \%$; $\mathrm{CTO}=61 \pm 1 \%$; V'E=40.6 $\pm 2.0 \mathrm{l} /$ min $(\mathrm{P}=\mathrm{NS}$, all comparisons NOT vs. placebo). Eight patients experienced ARAHE during the stay at $2048 \mathrm{~m}$ with placebo vs. one with NOT (Fisher's Exact Test, $\mathrm{P}<0.001$ ).

Conclusion: Altitude exposure impaired exercise endurance in patients with COPD due to arterial and cerebral hypoxemia. Nocturnal oxygen therapy prior to exercise did not improve performance but reduced ARAHE.

Grants: Lunge Zürich, SNSF

Disclosure: Nothing to disclose

\section{9 \\ Surveillance of Employees of the Swiss Reception Centers for Asylum Seekers for Latent Tuberculosis Infection}

J.-P. Zellweger ${ }^{1}$, A. Zellweger ${ }^{2}$, J.-M. Egger ${ }^{1}$, A. Koller-Doser ${ }^{3}$

${ }^{1} \mathrm{~TB}$ Competence Center, Swiss Lung Association, Berne,

${ }^{2}$ Meditest Analytical Laboratory, Lausanne, ${ }^{3}$ State Secretariat for

Migration, Berne, Switzerland

Settings: migrants claiming for asylum in Switzerland have to register in reception centers before they are allowed to entry in the country. Some of them may have active tuberculosis at entry and transmit it to other persons.

Aim: To assess the risk of tuberculosis infection among employees of 7 reception centers close to the Swiss border.

Method: Employees of the reception centers were offered a free IGRA test at hiring and at yearly interval. Employees with a positive test at hiring or during the surveillance were referred to a medical doctor for further examination.

Results: Between 2010 and 2018, 1494 tests were performed among 736 employees (402 men). A single test was performed in 484 persons, tests were repeated at least twice in 248 persons (4 missing informations). Twenty persons had a positive IGRA test at the first control, 2 persons converted from negative to positive, 2 persons had stable positive tests and 2 persons reverted from positive to negative. No case of active TB was observed among the employees.

Conclusions: The prevalence of latent TB infection among new employees is low $(20 / 484=4.1 \%)$. The risk of acquiring latent TB infection, defined as a conversion of IGRA from negative to positive, is low $(2 / 248$ persons $=0.8 \%$ or $2 / 1010$ repeated tests $)$. Yearly controls of IGRAs in the absence of documented exposure to TB seems unnecessary.

Disclosure: Nothing to disclose 


\section{9}

\section{Regulatory T Cells in Bleomycin Injured Mouse Lung}

$\underline{\text { S. Mutlu }}{ }^{1}$, K. Fytianos ${ }^{2}$, C. Wotzkow ${ }^{2}$, S. Steiner ${ }^{2}$, C. Ferrié2 T. Anna-Barbara ${ }^{2}$, A. Gazdhar ${ }^{2}$, F. Blank ${ }^{2}$

${ }^{1}$ Departement of Biomedical Research, University of Bern, ${ }^{2}$ Departement of Biomedical Research, Inselspital, Bern University Hospital, University of Bern, Bern, Switzerland

Introduction: Idiopathic pulmonary fibrosis (IPF) is a lethal disease of unknown etiology. It is characterized by progressive loss of alveolar epithelial integrity due to dysfunctional wound repair mechanisms. Multiple cell types play an important role in disease pathogenesis, however, the role of Immune cells specially regulatory T cells (Tregs) is not well defined. In the current study we aim to study immune cell homeostasis and the role of regulatory $\mathrm{T}$ cells in pathogenesis of lung fibrosis in a mouse model.

Methods: C57BL/6jrJ mice were administered $1.52 \mathrm{U} / \mathrm{kg}$ of bleomycin intratracheally and sacrificed at $7(n=3), 10(n=9)$ and $14(\mathrm{n}=6)$ days after bleomycin administration. Single-cell suspensions of lung parenchyma, bronchoalveolar fluid (BAL) and lung draining lymph nodes were analyzed by flow cytometry.

Results: Our data show a decrease in number of Tregs 7 days after bleomycin instillation. This decrease was followed by a partial recovery of Tregs at day 10 , followed by decrease at day 14 after bleomycin instillation in the lung, demonstrating a disrupted homeostasis of the pulmonary immune system. Other immune cells such as dendritic cells and macrophages also showed a similar pattern compared to Tregs during the course of bleomycin induced lung injury.

Conclusion: Bleomycin administration alters immune cell homeostasis in the mouse lung during the course of development of fibrosis.

Disclosure: Nothing to disclose

\section{6 \\ Change in 6-Minute Walk Distance during Pulmonary Rehabilitation is Associated with Improved Survival in Patients with Fibrotic Interstitial Lung Disease}

\author{
S.A.Guler ', S.A. Hur ${ }^{2}$, P. Brun ${ }^{3}$, M.K. Stickland ${ }^{4}$, L. Bovet ${ }^{3}$, \\ A. Holland ${ }^{5}$, J. Bondarenko ${ }^{5}$, N. Hambly ${ }^{6}$, J. Wald ${ }^{6}$, N. Makhdami ${ }^{6}$, \\ M. Kreuter ${ }^{7}$, R. Gloeckl ${ }^{8}$, I. Jarosch ${ }^{8}$, B. Tan ${ }^{2}$, K.A. Johannson ${ }^{9}$, \\ S.A.McBride ${ }^{9}$, K. de Boer ${ }^{10}$, K. Sun ${ }^{11}$, D. Assayag ${ }^{12}$, S. Bhatt ${ }^{13}$, \\ J. Morisset ${ }^{14}$, C. Garvey ${ }^{15}$, P. Camp ${ }^{2,16}$, C.J. Ryerson ${ }^{2,17}$ \\ ${ }^{1}$ Department of Pulmonary Medicine, Inselspital, Bern \\ University Hospital, University of Bern, Bern, Switzerland, \\ ${ }^{2}$ Department of Medicine, University of British Columbia, \\ Vancouver, BC, Canada, ${ }^{3}$ Berner Reha Zentrum \\ Heiligenschwendi, Heiligenschwendi, Switzerland, ${ }^{4}$ University \\ of Alberta, Edmonton, G.F. MacDonald Centre for Lung Health,
}

Edmonton, $\mathrm{AB}$, Canada, ${ }^{5}$ Monash University and Alfred Health, Melbourne, SA, Australia, ${ }^{6}$ Department of Medicine, Firestone Institute for Respiratory Health, McMaster University, Hamilton, ON, Canada, ${ }^{7}$ Center for Interstitial and Rare Lung Diseases, Thoraxklinik, University of Heidelberg and German Center for Lung Research, Heidelberg, ${ }^{8}$ Institute for Pulmonary Rehabilitation Research, Schoen Klinik Berchtesgadener Land, Schoenau am Koenigssee, Germany, ${ }^{9}$ Department of Medicine, University of Calgary, Calgary, AB, Canada, ${ }^{10}$ Department of Medicine, Division of Pulmonary Medicine and Critical Care, Stanford University, Stanford, CA, United States, ${ }^{11}$ Department of Medicine, University of Ottawa, Ottawa, ON, ${ }^{12}$ Department of Medicine, McGill University, Montreal, QC, Canada, ${ }^{13}$ Division of Pulmonary, Allergy, and Critical Care Medicine, University of Alabama at Birmingham, Birmingham, AL, United States, ${ }^{14}$ Département de Médecine, Centre Hospitalier de I'Université de Montréal, Montréal, QC, Canada, ${ }^{15}$ Pulmonary Rehabilitation and Sleep Disorders Center, University of California San Francisco, San Francisco, CA, United States, ${ }^{16}$ Department of Physical Therapy, University of British Columbia, ${ }^{17}$ Centre for Heart Lung Innovation, University of British Columbia, Vancouver, BC, Canada

Introduction: Pulmonary rehabilitation (PR) improves functional capacity in patients with fibrotic interstitial lung disease (ILD); however, the effect of PR on survival is unknown. The objective of this retrospective, cohort study was to determine whether improvement in 6-minute walk distance (6MWD) during $\mathrm{PR}$ is associated with better survival in patients with ILD.

Methods: Ten centers collected data from patients with fibrotic ILDs completing outpatient or inpatient PR. The association of $\triangle 6 \mathrm{MWD}$ with 1 - and 2-year survival was analyzed using logistic regression models with mixed effects accounting for clustering by center. Cox proportional hazards models with mixed effects were used to estimate time to death or lung transplantation. Odds ratios (OR) and hazard ratios (HRs) are reported per 10m/10\% $\triangle 6 \mathrm{MWD}$.

Results: 427 and 196 patients who attended inpatient and outpatient PR were included. The mean (SD) age was 69 (11) years, $63 \%$ were male, and 59\% ever-smokers, FVC was 69 (21) $\%$-predicted, and DLCO 47 (18) \%-predicted. Baseline 6MWD was 339 (133) with improvement by median 43 (IQR 2-85) meters after PR. Over a median observation period of 26 (IQR 10-60) months, 162 patients died and 24 had a lung transplantation. 1-, 2-, and 5 -year survival was $88 \%, 77 \%$, and $64 \%$. 6MWD increase $>30 \mathrm{~m}$ during PR was associated with prolonged time to death or lung transplantation, adjusted for age, sex, body mass index, FVC\%predicted, IPF diagnosis, and baseline 6MWD (HR 0.63, 95\%CI $0.44-0.90, \mathrm{p}=0.01) . \Delta 6 \mathrm{MWD}$ was associated with both $1-$ and 2-year survival (OR 0.92, 95\%CI 0.87-0.96, $\mathrm{p}=0.0007$, and OR 0.93, $95 \%$ CI $0.89-0.96, \mathrm{p}=0.0002)$, after adjustment for the above variables.

Conclusions: We demonstrate a significantly better survival in patients who had clinically important gains in $6 \mathrm{MWD}$ with $\mathrm{PR}$, compared to those who did not. These findings may suggest a potential survival benefit from PR in patients with fibrotic ILD.

Disclosure: Nothing to disclose 


\section{7 \\ Clinical Presentation and Analysis of Liquids Used by Patient with Probable E-Cigarette or Vaping Associated Lung Injury and Bronchiolitis Phenotype}

\author{
R. Hage ${ }^{1}$, M.M. Schuurmans ${ }^{1,2}$ \\ ${ }^{1}$ Pulmonology, University Hospital Zurich, Zurich, \\ ${ }^{2}$ Pulmonology, Cantonal Hospital Winterthur, Winterthur, \\ Switzerland
}

Introduction: A previously healthy 44-year-old female presented with severe dyspnea, cough, wheezing and dyspepsia. Her symptoms had started a few months ago after stopping smoking with electronic (e-) cigarettes containing nicotine. She had been diagnosed by her general practitioner with adult-onset asthma but did not respond well to inhaled fluticasone propionate/ formoterol and montelukast. Repetitive high dose systemic corticosteroid treatments did help initially, but not for the current exacerbation. Clinically she presented with a severe bronchiolitis with end-inspiratory squeaks. A this point in time she was using nicotine-free e-liquids. Spirometry showed a severe obstruction and arterial blood gas revealed a hypoxemia (pO2 $7.3 \mathrm{kPa})$. The diagnosis of a probable case of e-cigarette or vaping associated lung injury (EVALI) was suspected. The patient markedly improved on therapy with a macrolide, steroids and discontinuation of e-cigarette consumption, as recommended. Chest computed tomography after initiation of treatment showed residual groundglass opacities and air trapping. The exact pathophysiology of EVALI is unknown, however, vitamin $\mathrm{E}$ and the flavorants diacetyl and 2,3-pentanediol found in e-cigarettes and in BAL fluid samples could be causative agents.

Methods: Our case is discussed in the context of the current literature reporting on EVALI. Two commercially available fruitflavored e-liquids purchased from the same E-cigarette supplier as the patient are currently being investigated for potential components implicated in EVALI.

Results: The bronchiolitis phenotype has rarely been described in EVALI cases to date. We describe such a case in detail. One life-threatening case of EVALI associated bronchiolitis in a 17 -year-old male has been published before causing near-fatal hypercapnic respiratory failure requiring intubation and extracorporeal membrane oxygenation (Landmann ST. CMAJ 2019). Additionally, bronchiolitis as important histopathological finding in lung biopsies of EVALI patients has been described (Butt YM. NEJM 2019). The analysis results of the e-liquids are pending.

Conclusion: A probable case of EVALI with a bronchiolitis phenotype is presented and will be discussed in the context of known evidence on EVALI including the analysis results of the e-liquids.

Disclosure: Nothing to disclose

\section{7 \\ Squeaks in Hypersensitivity Pneumonitis: Prevalence, Clinical Correlates and Diagnostic Value}

E. Diamanti ${ }^{1}$, C. Daccord ${ }^{1}$, A. Casutt ${ }^{1}$, A. Christe ${ }^{1}$, G. Humair ${ }^{1}$, N. Lambert ${ }^{1}$, B. Lechartier ${ }^{1}$, C. Perez Valdes ${ }^{1}$, M. Faouzi ${ }^{2}$, R. Lazor ${ }^{1}$

${ }^{1} \mathrm{CHUV},{ }^{2}$ Center for Primary Care and Public Health, Division of

Biostatistics, University of Lausanne, Lausanne, Switzerland

Introduction: Squeaks (SQ) are high-pitched end-inspiratory "musical" sounds rarely observed at chest auscultation. They have been reported in hypersensitivity pneumonitis (HP), but their prevalence and correlation with clinical features remain unknown. The goals of this study were to determine: a) the prevalence of SQ in $\mathrm{HP}, \mathrm{b}$ ) the associations of SQ with clinical findings, and c) the diagnostic value of SQ to distinguish HP from nonspecific interstitial pneumonia (NSIP) and usual interstitial pneumonia (UIP).

Methods: Since 2017, patients with interstitial lung disease (ILD) were routinely assessed for the presence or absence of SQ at chest auscultation by $\geq 2$ observers, including $\geq 1$ ILD expert. In August 2019, medical records were reviewed retrospectively. Cases were divided in 2 groups: HP group according published diagnostic criteria (Vasakova 2017), and control group (NSIP and UIP). Diagnosis was assessed independently of the SQ status. The presence or absence of SQ was assessed independently of the diagnosis.

Results: 108 patients were included: $50 \mathrm{HP}$ and 58 controls (31 NSIP, 27 UIP). The prevalence of SQ was $48 \%$ in HP, and $16 \%$ in controls. In HP, SQ were associated with female gender (OR 3.78, $\mathrm{p}=0.026$ ), lower FEV1 (OR 0.90, $\mathrm{p}=0.002$ ), lower FVC (OR $0.93, \mathrm{p}=0.002)$ and higher RV/TLC (OR 1.04, $\mathrm{p}=0.005)$. The value of SQ alone for the diagnosis of HP was: sensitivity $48 \%$, specificity $85 \%$, positive predictive value $73 \%$, negative predictive value $65 \%$. SQ alone allowed to correctly classify $68 \%$ of patients. In univariate analysis, SQ were significantly associated with a diagnosis of HP (OR 5.02, $\mathrm{p}<0.0001)$.

Conclusions: SQ are found in half of patients with HP, and are associated with female gender, lower lung volumes and air trapping. SQ are significantly associated with the diagnosis of HP, as compared to NSIP and UIP. Their inclusion in HP diagnostic criteria should be considered.

Disclosure: Nothing to disclose 


\section{2}

\section{Antifibrotic Activities of SDF-1 in an Animal Model of Lung Fibrosis}

\section{A. Gazdhar ${ }^{1,2}$, K. Fytianos ${ }^{1,2}$, R. Schliep ${ }^{3}$, S. Mykoniati ${ }^{4}$, P. Khan ${ }^{5}$,} K. Hostettler ${ }^{5}$, L. Knudsen ${ }^{3}$, T. Geiser ${ }^{1,2}$

'Department of Pulmonary Medicine, University Hospital Bern, ${ }^{2}$ Department of Biomedical Research, University of Bern, Bern, Switzerland, ${ }^{3}$ Institute of Anatomy, Hannover Medical School, Hanover, Germany, ${ }^{4}$ Department of Internal Medicine, Cantonal Hospital of Jura, Delemont, ${ }^{5}$ Department of Biomedicine, University Hospital Basel, Basel, Switzerland

Introduction: Idiopathic pulmonary fibrosis (IPF) is a progressive interstitial lung disease and associated with high mortality. Excessive deposition of extracellular matrix by activated myofibroblasts in the alveolar interstitial space leads to scar formation that hinders gas exchange. Stromal derived growth factor (SDF-1) plays important role in tissue repair and remodelling and may therefore act as a novel antifibrotic mediator. We therefore investigated the effect of SDF-1 on lung injury and fibrosis using the bleomycin injured rat lung model.

Material and methods: Adult male Fischer rats (F344) were used. Invivo electroporation-mediated SDF-1 gene transfer was performed (pSDF-1) and after 7 days compared to control (electroporation of empty vector $(\mathrm{EV})$.

Results: In presence of SDF-1, total collagen was reduced $(252.3 \pm 50.1$ vs $554.5 \pm 97.4 \mathrm{ug} / \mathrm{mg}$ of lung tissue), fibrotic histology was improved (quantified by decrease in the modified Ashcroft's score in SDF-1 2.26 \pm 0.74 compared to EV 3.03 \pm 0.90 ), collagen fibrils were reduced and TNF- $\alpha$ mediated apoptosis of myofibroblasts was induced ( $36.09 \pm 8.36$ vs $41.96 \pm 6.62)$. In addition, SDF- 1 overexpression increased alveolar epithelial cell (AEC) numbers and AEC proliferation in vivo (58.91 $\pm 2.01 \%$ vs $33.06 \pm 3.19 \%$ compared to EV) and induced AEC migration in vitro as measured by alveolar epithelial wound healing $(100 \pm 0 \%$ vs $8.76 \pm 5.30 \%$ in 24 hours).

Conclusion: Our study demonstrates a new antifibrotic effect of SDF-1 that is due to modulating lung (myo)fibroblasts and AEC.

Disclosure: Supported by a SINERGIA Grant by the Swiss National Science Foundation SNF

\section{7 \\ Prognostic Value of Bronchoalveolar Lavage Fluid CD103+CD4+/CD4+ Ratio in Sarcoidosis}

C. Schilt ${ }^{1}$, C. Daccord ${ }^{2}$, M.-E. Muller ${ }^{2}$, M. Bongiovanni ${ }^{3}$, M. Faouzi ${ }^{4}$, R. Lazor $^{2}$

${ }^{1}$ Internal Medicine Department, Broye Intercantonal Hospital, Payerne, ${ }^{2}$ Respiratory Medicine Department, Lausanne University Hospital and University of Lausanne, ${ }^{3}$ Synlab Pathologie, ${ }^{4}$ Division of Biostatistics, University of Lausanne, Lausanne, Switzerland

Introduction: The CD103+ integrin is an adhesion molecule found on $>95 \%$ of intra-epithelial mucosal lymphocytes, but on $<2 \%$ of circulating lymphocytes. The proportion of CD $4+$ lymphocytes expressing the CD103+ integrin (CD103+CD4+/CD4+ ratio) in bronchoalveolar lavage fluid (BALF) is low in sarcoidosis compared to other interstitial lung diseases, but the difference is insufficient to have diagnostic value in clinical practice. To determine whether BALF CD103+CD4+/CD4+ ratio at presentation conveys prognostic information in sarcoidosis, we retrospectively examined associations between BALF CD103+CD4+/CD4+ ratio and clinical and imaging characteristics at diagnosis and during follow-up.

Methods: Cases of sarcoidosis with BALF flow cytometry performed at the Lausanne university hospital were retrospectively reviewed. Demographic data, lung function and imaging characteristics at diagnostic and last visit, BALF cytology, extrapulmonary organ involvement, the need for systemic treatment, and the clinical phenotype were analyzed.

Results: 115 patients were included (men 63\%, median age 48 years). At diagnosis, $47 \%$ had radiographic stage I, $49 \%$ had stage II, and $4 \%$ had stage III-IV. At last visit, 30\% had normal chest X-ray, 37\% had stage I, $20 \%$ had stage II, and 13\% had stage III-IV. The median BALF lymphocyte count was $43 \%$ and the median $\mathrm{CD} 103+\mathrm{CD} 4+/ \mathrm{CD} 4+$ ratio was $17 \%$. A higher CD103+CD4+/ $\mathrm{CD} 4+$ ratio was associated with a higher radiographic stage at diagnosis $(\mathrm{p}=0.017)$ and last visit $(\mathrm{p}<0.0001)$, and the presence of fibrosis at imaging at diagnosis $(\mathrm{p}=0.043)$ and last visit $(\mathrm{p}=0.032)$. Besides an inverse correlation with forced vital capacity at diagnosis $(\beta=-0.16, p=0.031)$, no consistent association was found between the BALF CD103+CD4+/CD4+ ratio at diagnosis and lung function parameters at diagnosis and last visit.

Conclusion: In sarcoidosis, a higher BALF CD103+CD4+/ $\mathrm{CD} 4+$ ratio is associated with a higher radiographic stage and presence of fibrosis at imaging, both at diagnosis and at last visit. BALF CD103+CD4+/CD4+ ratio has prognostic value in sarcoidosis.

Disclosure: Nothing to disclose 


\section{8}

\section{Fatigue in Sarcoidosis: Are there Correlations with Inflammatory Biomarkers?}

\author{
N. Domon' ${ }^{1}$, S. Chatelain' ${ }^{2}$, C. Daccord ${ }^{3}$, A. Ogna ${ }^{3}$, L. Mercier ${ }^{3}$, \\ E. Bernasconi ${ }^{3}$, C. Ribi ${ }^{4}$, L. Charvoz ${ }^{2}$, L.P. Nicod ${ }^{3}$ \\ ${ }^{1}$ Medical School of the Faculty of Biology and Medicine, \\ University of Lausanne (UNIL), ${ }^{2}$ University of Applied Sciences \\ and Arts Western Switzerland, Haute Ecole de Travail Social \\ et de la Santé, ${ }^{3}$ Division of Respiratory Medicine, ${ }^{4}$ Division of \\ Immunology and Allergology, Centre Hospitalier Universitaire \\ Vaudois (CHUV), Lausanne, Switzerland
}

Introduction: Fatigue is a highly prevalent non-specific symptom associated with sarcoidosis, which can impact daily activities, quality of life and mental health. Current knowledge suggests an association between fatigue and a persistent inflammatory activity of sarcoidosis. However, no inflammatory parameter has so far been causally associated with the presence and severity of fatigue in sarcoidosis. The aim of this study was to look for correlations between fatigue and inflammatory biomarkers in patients with sarcoidosis.

Methods: Inclusion criteria were a diagnosis of sarcoidosis and patient-reported fatigue not explained by another typical cause of fatigue. Fatigue status was assessed at 0,6 and 12 months via the Fatigue Assessment Scale (FAS) questionnaire, providing physical, mental and total scores of fatigue. Blood sample was taken at the same timepoints to measure serum concentration of various inflammatory parameters, including IL-1 $\beta$, IL-6, IL-8, TNF- $\alpha$, CRP, ferritin and ESR. Pearson's correlation coefficient and t-tests were applied.

Results: Based on a preliminary analysis of 9 out of 24 patients, FAS total score was abnormal ( $\geq 22$ pts) in $76 \%$ of assessments with a mean of $28.2 \pm 8.5$ pts and fatigue was "extreme" ( $\geq 35$ pts) in $20 \%$. Mean physical fatigue score (15.4 $\pm 5.1 \mathrm{pts})$ was higher than mental score $(12.7 \pm 4.4 \mathrm{pts})(\mathrm{p}=0.025)$. Patients with systemic sarcoidosis had higher total $(\mathrm{p}=0.016)$ and physical $(\mathrm{p}=0.007)$ FAS score compared to patients with thoracic involvement only. Ferritin was positively correlated with FAS total score $(\mathrm{p}=0.002 ; \mathrm{R}=0.598)$. Except for a negative correlation with IL-8 serum level $(\mathrm{p}<0.01$; $\mathrm{R}=-0.55$ ), there was no correlation between other cytokines, CRP and ESR and fatigue.

Conclusions: This preliminary analysis on 9 patients suggests a significant positive correlation between ferritin serum concentration and fatigue in sarcoidosis. The data of 15 additional patients are currently being analysed to confirm these results.

Disclosure: Nothing to disclose

\section{1 \\ Diagnostic Value of Cryobiopsy in Patients with Interstitial Lung Diseases Integrating Bronchoalveolar Lavage, Radiologic and Clinical Data}

K.E. Hostettler ${ }^{1}$, S. Savic Prince ${ }^{2}$, L. Bubendorf ${ }^{2}$, P. Grendelmeier ${ }^{1}$, K. Jahn ${ }^{1}$, D. Stolz ${ }^{1}$, J. Bremerich ${ }^{3}$, M. Tamm ${ }^{1}$

${ }^{1}$ Clinics of Respiratory Medicine, ${ }^{2}$ Institute for Pathology, ${ }^{3}$ Department of Radiology, University Hospital Basel, Basel, Switzerland

Background: Transbronchial cryobiopsy (cTBB) in the diagnostic evaluation of patients with interstitial lung diseases (ILD) is expected to considerably reduce the need for surgical lung biopsy.

Objective: To evaluate the diagnostic value of cTBB in combination with bronchoalveolar lavage (BAL), radiologic and clinical data in patients with ILD.

Methods: Between 8/2015 and 1/2020 patients with ILD who did not have a CT scan showing: i) typical UIP-pattern, ii) predominant ground-glass opacities, iii) findings suggestive of sarcoidosis, or iv) had non-diagnostic conventional transbronchial forceps biopsy, underwent cTBB. Histological findings were integrated into the multidisciplinary discussion (MDD) and a diagnostic consensus was sought.

Results: A total of 100 patients underwent cTBB. In 88/100 patients $(88 \%)$, cTBB was representative with diagnostic findings in $44 / 88$ and non-specific histological findings in 44/88 patients (50\% each). In 25/44 with non-specific findings (57\%), a consensus diagnosis was reached after MDD integrating BAL, radiologic and clinical data; 10 of the remaining 19 patients with non-specific findings were referred to surgical lung biopsy (SLB). In 12/100 patients $(12 \%)$ cTBB was not representative and three of these patients were also referred to SLB. In 9/13 patients (69\%) SLB was diagnostic. Complications of cTBB included pneumothorax (15\%) and locally controlled bleeding (23\%).

Conclusions: The diagnostic yield of cTBB was 69\%: 44\% of CTBB were diagnostic based on histology alone and an additional $25 \%$ provided non-specific, but valuable findings allowing a consensus diagnosis after MDD. Our data demonstrate that the diagnostic value of $\mathrm{CTBB}$ is high if combined with $\mathrm{BAL}$, radiologic and clinical data.

Disclosure: Nothing to disclose 


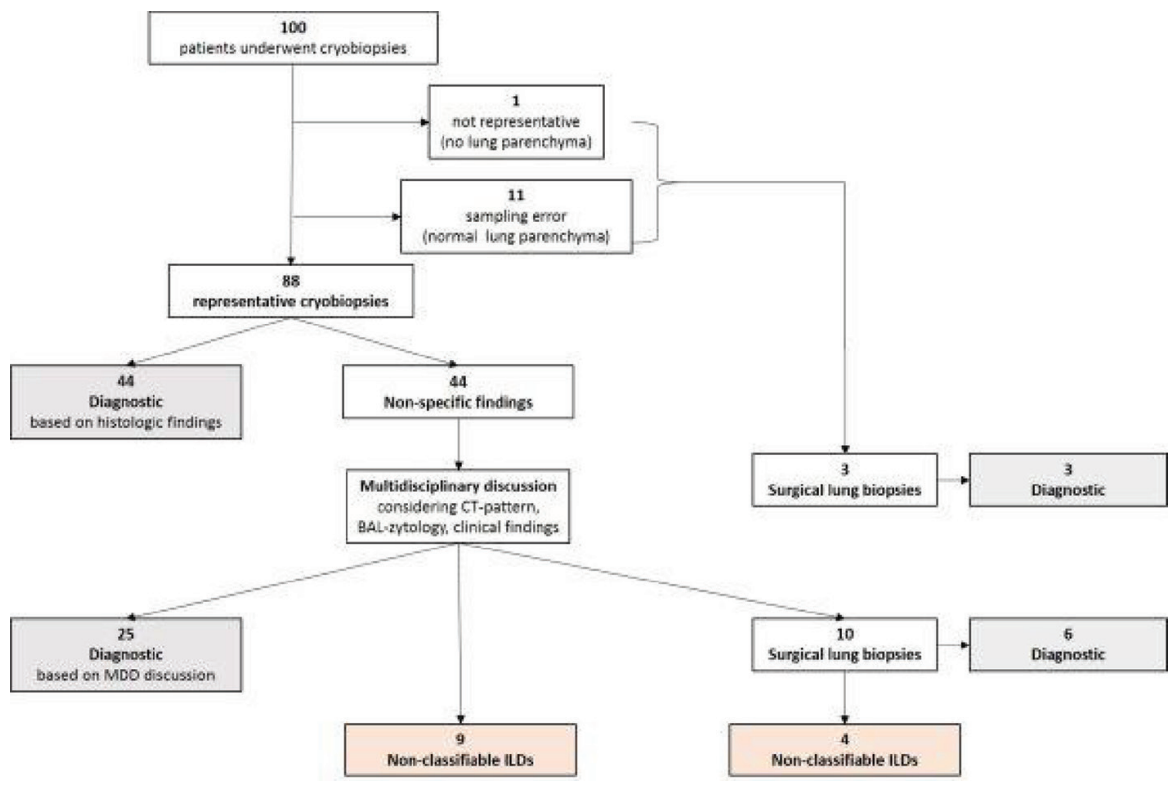

Fig. 1. Course of patients undergoing cTBB due to ILD (for Abstract 261).

\section{0 \\ Fibrosis-Specific Stem-Like Cells in Peripheral IPF Lung: Characteristics and Potential Role}

P. Khan ${ }^{1}$, J. Roux ${ }^{2,3}$, S. Savic Prince ${ }^{4}$, L. Knudsen ${ }^{5,6}$, D. Jonigk ${ }^{6,7}$,

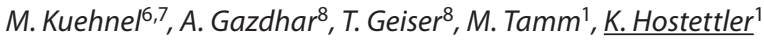

${ }^{1}$ Department of Biomedicine and Clinics of Respiratory Medicine, ${ }^{2}$ Department of Biomedicine, University Hospital Basel, ${ }^{3}$ Swiss Institute of Bioinformatics, ${ }^{4}$ Institute for Pathology, University Hospital Basel, Basel, Switzerland, ${ }^{5}$ Institute of Functional and Applied Anatomy, Hannover Medical School, ${ }^{6}$ Biomedical Research in Endstage and Obstructive Lung Disease Hannover (BREATH), Center for Lung Research, ${ }^{7}$ Institute of Pathology, Hannover Medical School, Hannover, Germany, ${ }^{8}$ Department of Pulmonary Medicine, University Hospital Bern, Bern, Switzerland

Rationale: In idiopathic pulmonary fibrosis (IPF) alveolar architecture is disturbed, displaying fibrotic remodelling, loss of alveolar epithelial cells and the presence of atypical airway epithelial cells. We recently cultured fibrosis-specific stem-like cells from peripheral lung tissue and demonstrated significant anti-fibrotic effects of their secretome. Here we aimed to further characterize these cells and define their potential role in IPF.

Methods: Cells were cultured from fibrotic lung tissue and analysed by bulk or single cell RNA-sequencing (scRNA-seq), TaqMan-PCR or immunofluorescence (IF).
Results: scRNA-seq demonstrated an overall homogeneity and epithelial origin of the stem-like cells. scRNA-seq displayed that stem-like cells were overall homogeneous and mostly composed of cells from epithelial origin. The majority of epithelial cells expressed basal cell markers (KRT5, KRT17, TP63, ITGB4), of which a fraction of these co-expressed mesenchymal (VIM, FN1, CDH2), alveolar (SLC34A2, ABCA3, LPCAT1, EMP2, HOPX) and/or secretory epithelial cell marker (SCGB1A1, MUC4). Cells from IPF versus other ILD patients revealed significant differences in their transcriptome.

Conclusion: Stem-like cells show close transcriptomic similarities to airway basal cells, with a fraction of cells co-expressing mesenchymal, alveolar and/or secretory cell markers. The expression of alveolar and secretory cell marker likely indicates the cells capacity to differentiation towards these cell linages. Stem-like cells display a disease specific transcriptome, possibly induced by their specific microenvironment. A better understanding of the cells origin and contribution to IPF pathogenesis, may help to define new drug targets for IPF.

Disclosure: Funded by a Sinergia grant by the Swiss National Research Foundation and a grant by the SGP/Lungenliga. 


\section{Lung Cancer, Interventional Endoscopy}

90

\section{Endobronchial Microcoil Fiducial Markers Insertion for Stereotactic Lung Radiation Therapy; A Simple, Rapid and Safe Route}

\author{
$\underline{\text { A. Casutt }}{ }^{1}$, L. Noirez ${ }^{1}$, C. Beigelman-Aubry ${ }^{2}$, M. Bernasconi ${ }^{1,3}$, \\ R. Kinj ${ }^{4}$, A.-D. Durham ${ }^{4}$, C. Von Garnier ${ }^{1}$, A. Lovis ${ }^{1}$
}

${ }^{1}$ Pulmonology, ${ }^{2}$ Radiology, Centre Hospitalier Universitaire Vaudois, Lausanne, ${ }^{3}$ Pulmonology, Ospedale San Giovanni Bellinzona, Bellinzona, ${ }^{4}$ Radiation Oncology, Centre Hospitalier Universitaire Vaudois, Lausanne, Switzerland

Introduction: Stereotactic body radiation therapy (SBRT) is an alternative treatment for early stage inoperable lung cancer. Successful ablative treatment depends on the precise delivered radiation dose on the target. Metallic fiducial markers (FMs) allow tumor tracking by CyberKnife ${ }^{\bowtie}$, increasing precision. Transthoracic FMs insertion causes a high number of pneumothorax, endovascular insertion is a long procedure, and endobronchial linear-gold FMs insertion has a high migration rate compromising the tracking efficacy.

Therefore, we assessed a new endobronchial insertion technique of spiral microcoil FMs (m-FMs), commonly used for endovascular embolization procedures.

Methods: All patients from 10.2015 to 11.2019 with at least one peripheral pulmonary lesion $(\mathrm{PPL})<25 \mathrm{~mm}$ treated at our hospital by $\mathrm{m}$-FMs (Cook-Medical or Terumo) endobronchial placement were retrospectively evaluated. Transbronchial biopsies (TBB) were performed if the PPL was visible by the radial-endobronchial ultrasound (R-EBUS). In patients deemed too fragile by the local oncological multidisciplinary meeting, only m-FMs insertion was performed. The m-FMs tracking rate for Cyberknife ${ }^{\oplus}$, the migration rate at planification CT-scan before SBRT delivery, complications and procedure duration were described.

Results: 52 consecutive patients were treated during 55 procedures and $207 \mathrm{~m}$-FMs were placed for 68 PPL. Forty-three procedures were performed using local anesthesia (78\%). At least one m-FM was not in the expected segment for 9 PPL (13\%) representing a migration rate of $8 \%$ (16 m-FMs). Among 7/16 migrated $\mathrm{m}$-FMs, the target PPL was in a previously irradiated area. R-EBUS was employed for 33/68 PPL and TBB could be performed in 9 cases (13\%). Target tracking by Cyberknife ${ }^{\oplus}$ was possible for $64 / 68$ PPL (tracking rate 94\%). No complication occurred and the median procedure duration was 31.5 minutes.

Conclusions: Endobronchial m-FMs placement for PPL is rapid and safe with a low migration rate allowing accurate SBRT delivery. In previously irradiated areas, larger m-FMs may be a better option with less migration potential.

Disclosure: Nothing to disclose

\section{1 \\ Development of an Animal Model to Induce Airway Stenosis and the Placement of a 3D Printed Airway Stent}

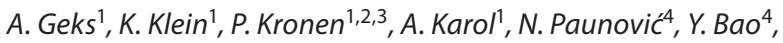
F. Coulter ${ }^{5}$, F. Rüber ${ }^{6}$, N. Kleger ${ }^{5}$, A. Studart ${ }^{5}$, J.-C. Leroux 4 , B. von Rechenberg $^{1,3}, \underline{D . F r a n z e n}^{6}$

'University of Zurich, Musculoskeletal Research Unit, Zurich, ${ }^{2}$ Veterinary Anaesthesia Services-International, Winterthur, ${ }^{3}$ University of Zurich, Center for Applied Biotechnology and Molecular Medicine, ${ }^{4}$ ETH Zurich, Institute of Pharmaceutical Sciences, ${ }^{5}$ ETH Zurich, Department of Materials, ${ }^{6}$ University Hospital Zurich, Department of Pulmonology, Zurich,

Switzerland

Introduction: Central airway obstruction can be caused by several benign or malignant conditions and has a huge impact on the quality of life. Therefore, bronchoscopic recanalization with or without airway stenting is the treatment of choice in most cases. However, airway stenting is not without harms due to re-stenosis and stent migration. Furthermore, there is only a limited choice of commercial stents available, which may not always fit the individual anatomy.

A personalized and drug-eluting stent, which degrades after the successful treatment of the stenosis could possibly solve these problems. To investigate such a device, a safe and reproducible animal model for induction of airway stenosis is needed.

Methods: Six female New Zealand White rabbits were anesthetized and underwent bronchoscopy, followed by circumferential brushing of the tracheal wall in order to induce a cicatricial airway stenosis.

Stenosis development was recorded via CT-scans and bronchoscopy.

After sacrifice, the stenotic tracheas were harvested and histologically analyzed.

Furthermore, within the framework of this project a prototype $3 \mathrm{D}$-printed, radiopaque and biodegradable stent made of poly (D,L-lactide-co- $\varepsilon$-caprolactone) was implanted into the healthy trachea of a rabbit to evaluate its biocompatibility using a custommade applicator-device.

Results: Stenosis could be proven in all animals. CT FollowUps over 4-6 weeks showed the maximum narrowing after week $2-4$, and stenosis ranged between $27 \%$ and $64 \%$, showing a gradual decrease over time.

Histologically, the stenotic area revealed tissue hypertrophy caused by accumulation of inflammatory cells, proliferation of fibroblasts with matrix deposition in all treated animals (Fig. 1).

The prototype stent could successfully be implanted and visualized by X-ray and CT imaging.

Conclusion: Despite the gradual decrease of stenosis over time, this model provides the opportunity to induce tracheal stenosis. In addition, the biocompatibility of a 3D printed stent and its ability to prevent airway obstruction can be evaluated.

Disclosure: This study was funded by the Sinergia program from the SNSF (grant No. 177178) 
Fig. 1A. Trachea cross section $27 \mathrm{~d}$ FollowUp, Stenotic area (arrows), overview, Van Gieson Elastica (for Abstract 111).

Fig. 1B. Trachea cross section 27 d Follow-Up, Stenotci area (arrows), detailed, Van Gieson Elastica (for Abstract 111).
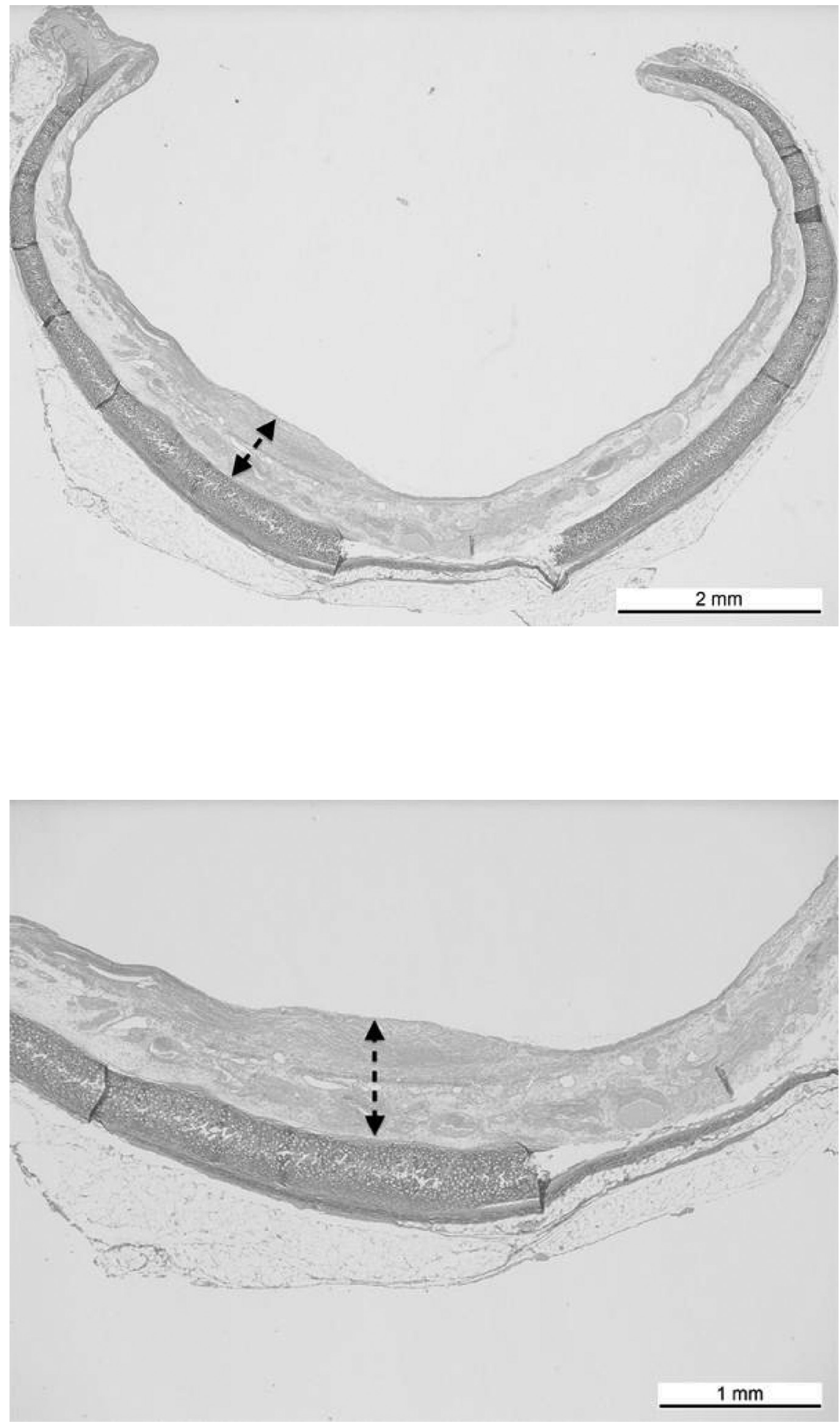


\section{0}

\section{Transbronchial Lung Cryobiopsies: A Single-Center Interventional Pulmonology Experience with First 25 Cases}

\section{Noirez, C. Von Garnier, A. Lovis}

Service de Pneumologie, Centre Hospitalier Universitaire Vaudois (CHUV), Lausanne, Switzerland

Introduction: Transbronchial lung cryobiopsy (TBCB) has proven its usefulness in diagnostic approach to interstitial lung disease (ILD) with a good diagnostic yield when associated with clinical history and radiological imaging. Severe bleeding and pneumothorax are the most important procedure-associated complications and may be life threatening. Based on our experience we are assuming that safety of TBCB depends on standardized protocol. This seems to be consistent with recently published expert guidelines.

Materials and methods: We reviewed retrospectively all procedures with TBCB in our tertiary care center performed with the same protocol including systematically two pulmonologists by procedure who have undergone training in interventional pulmonary procedures, rigid bronchoscopy, flexible bronchoscope, a bronchial blocker inflated to wedge the biopsied segment after each TBLB and fluoroscopy for optimal cryoprobe placement.

Results: A total of 25 patients underwent TBCB under general anesthesia (GA) between October 2017 and January 2020. The median duration was 40 minutes including examination of the tracheobronchial tree, bronchoalveolar lavage and five TBCB with a freezing time from 3 to 5 seconds by sample according to the probe size selection ( 1.9 or $2.4 \mathrm{~mm}$ ). Severe bleeding occurred in 1 case because the balloon was dislodged during the procedure, pneumothorax occurred in 4 cases (16\%) with 3 of whom (12\%) required an intercostal pleural catheter and no cases needed surgical operation. Histological diagnosis was obtained in 21 (84\%).

Conclusion: Our preliminary experience confirms the feasibility and safety of performing TBCB with a standardization of procedure including GA, secured airway, fluoroscopy guided TBCB, prophylactic measure to manage bleeding. This reproducible procedure is easily achievable from the first biopsies. Our diagnosis yield is consistent with previous meta-analysis.

Disclosure: Nothing to disclose

\section{Vascular Lung Diseases}

\section{7 \\ Transition from IV Treprostinil to PO Selexipag in Patients with Severe Primary Pulmonary Hypertension: Case Report and Review of the Literature}

S. Svab, J. Pichler Hefti, S. Krebser, L. Meier Laub, M. Schwerzmann

Inselspital, Bern University Hospital, University of Bern, Bern, Switzerland

Introduction: Synthetic Prostacyclins are effective vasodilators in patients with pulmonary hypertension. One disadvantage in their use is the continuous parenteral administration route necessary for some of these drugs, which is challenging for the patient and has the potential for device-related infections, catheter-related embolism and mechanical complications. Selexipag, an oral non-prostanoid prostacyclin-receptor agonist, was specifically developed to overcome this limitation. The clinical effectiveness of replacing a parenteral Prostacyclin with Selexipag has not been systematically investigated so far. Case reports have been described, with mixed results regarding this transition. We aim to describe our experience in one patient and summarize the currently existing literature.

Method: We report a patient with idiopathic pulmonary arterial hypertension and stable clinical course under a triple pulmonary vasodilator therapy, including intravenously administered Treprostinil, who required transition of parenteral Prostacyclin therapy to Selexipag after multiple device-related complications. We describe his clinical course in the 3 years after the transition (Figure 1).

We also report a review of the literature dealing with the transition from parenteral Prostacycline to Selexipag, obtained searching the literature with the keywords "Transition", "Prostacyclin" and "Selexipag" and then scanning the identified articles for further references.

Results: In the present patient, the clinical and hemodynamic course on parenteral Treprostinil was more favourable than after the transition to Selexipag (Figure 2, Table 1), despite the use of an off-label high dose of this oral drug. The clinical status improved again after the therapy was switched back to Treprostinil. The literature on this topic is divergent (Table 2).

Conclusion: In our experience, the transition from Treprostinil to Selexipag was feasible without acute right heart decompensation, and it allowed us to gain some time during the acute infection. In the long term though, despite using a high dose, the clinical response was less favourable on Selexipag than under Treprostinil. The reasons for that are speculative. Our experience emphasizes the importance of a clinical follow up after the switch between the two drugs. More systematic studies are needed to better understand the relative roles of Treprostinil and Selexipag and to better predict the response to the two drugs.

Disclosure: Nothing to disclose 
Fig. 1. Timeline of events during the patient's history. IV: Intravenous; SC: subcutaneous; PO: per os (for Abstract 87).
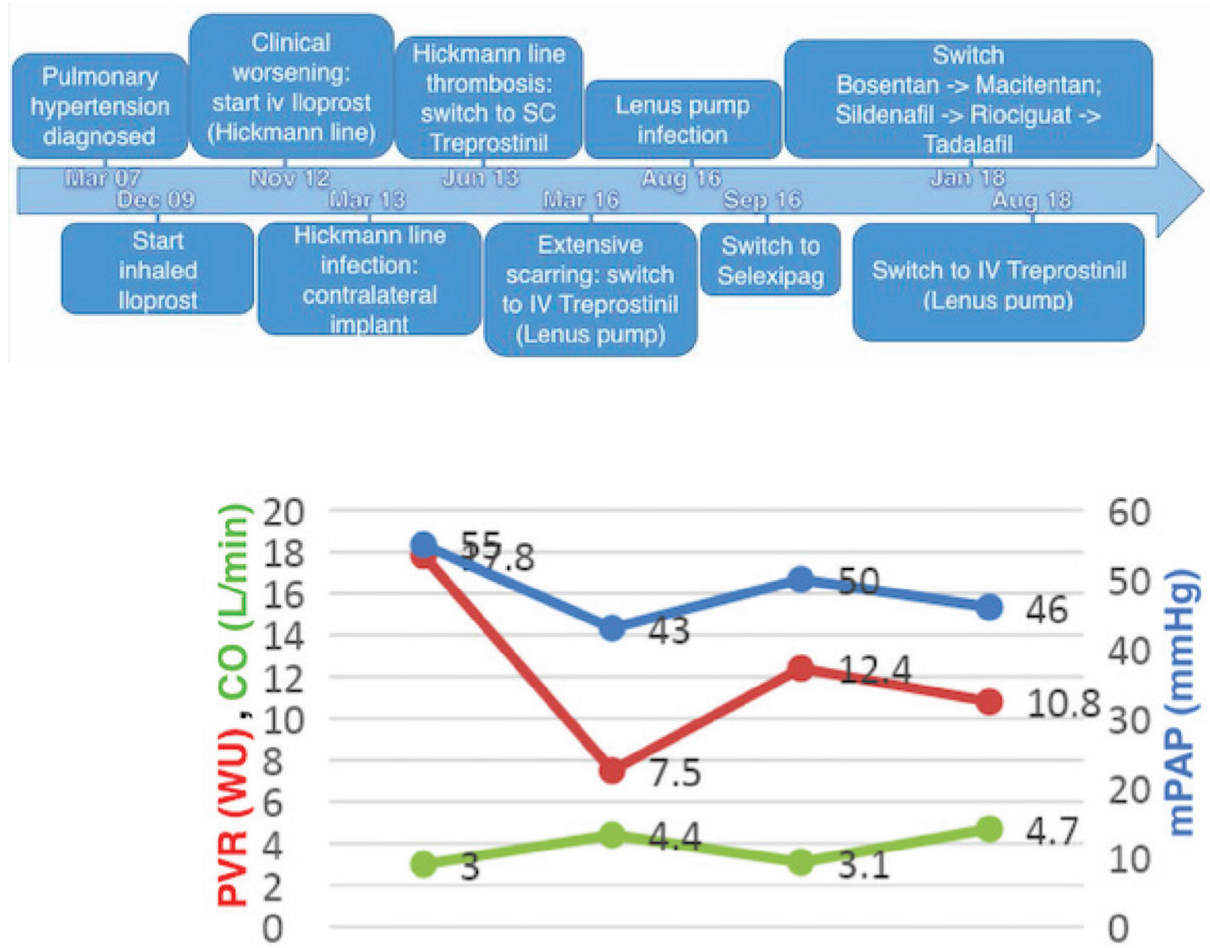

Fig. 2. Changes in invasive hemodynamics. PVR: pulmonary vascular resistance; CO: cardiac output; mPA (for Abstract 87).

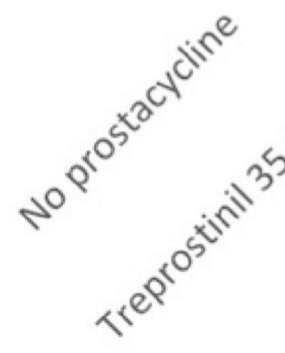

PVR (WU)

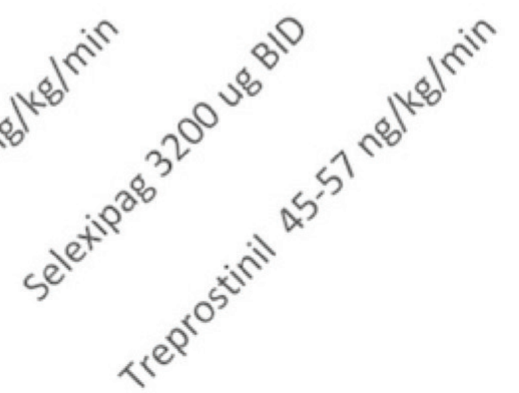

Table 1. Summary of patient's parameters. 6MWD: 6-Minute Walking Distance; mLAP: mean left atrial pressure; Qp/Qs: pulmonary/ systemic flow (for Abstract 87)

\begin{tabular}{|c|c|c|c|c|}
\hline & Mar 07 & Aug 16 & Jun 18 & Jan - Jun 19 \\
\hline Therapy & None & $\begin{array}{l}\text { Treprostinil } 35 \mathrm{ng} / \mathrm{kg} / \mathrm{min} \text {, } \\
\text { Bosentan } 125 \mathrm{mg} \mathrm{BID} \text {, } \\
\text { Sildenafil } 50 \mathrm{mg} \text { TID, } \\
\text { Imatinib } 200 \mathrm{mg} \text { OD. }\end{array}$ & $\begin{array}{l}\text { Selexipag } 3200 \mathrm{ug} \text { BID, } \\
\text { Macitentan } 10 \mathrm{mg} \text { OD, } \\
\text { Riociguat } 2.5 \mathrm{mg} \text { TID, } \\
\text { Imatinib } 200 \mathrm{mg} \text { OD. }\end{array}$ & $\begin{array}{l}\text { Treprostinil } 45 \text { - } 57 \mathrm{ng} / \mathrm{kg} / \mathrm{min} \text {, } \\
\text { Macitentan } 10 \mathrm{mg} \mathrm{OD} \text {, } \\
\text { Tadalafil } 40 \mathrm{mg} \text { OD, } \\
\text { Imatinib } 200 \mathrm{mg} \text { OD. }\end{array}$ \\
\hline 6MWD (m) & 482 & 570 & 560 & 555 \\
\hline NTproBNP (pg/mL) & 1280 & 859 & 784 & 980 \\
\hline mPAP $(\mathrm{mmHg})$ & 55 & 43 & 50 & 46 \\
\hline mLAP (mmHg) & 7 & 10 & 12 & 12 \\
\hline PVR (WU) & 17.8 & 7.5 & 12.4 & 10.8 \\
\hline $\mathrm{CO}(1 / \mathrm{min})$ & 3 & 4.4 & 3.1 & 4.7 \\
\hline Qp/Qs & 0.8 & 0.8 & 0.6 & 0.7 \\
\hline TAPSE (mm) & 21 & 34 & 23 & 27 \\
\hline
\end{tabular}


Table 2. Review of the literature about the switch from parenteral Treprostinil to oral Selexipag. PAH: pulmonary arterial hypertension (for Abstract 87)

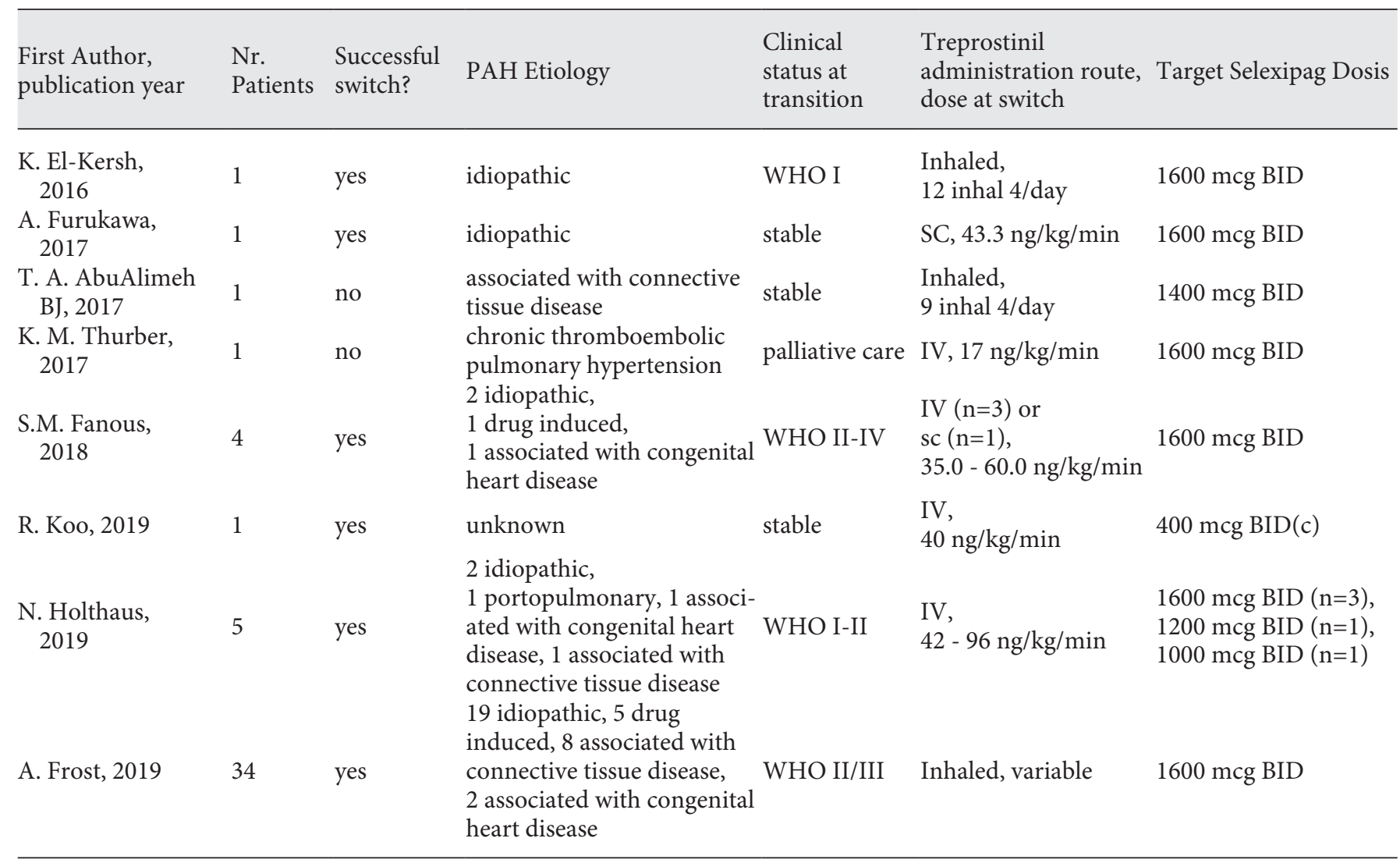

\section{0 \\ Impact of a Volume Challenge on Hemodynamics and Prognostic Implications in Patients with Severe Aortic Stenosis}

\section{Weber, H. Rickli, D. Weilenmann, P.K. Haager, L. Joerg, F. Rohner, \\ P. Ammann, J. Chronis, J. Rigger, M.T. Maeder}

Kantonsspital St. Gallen, St. Gallen, Switzerland

Background: Use of a volume challenge has been proposed to unmask pulmonary hypertension $(\mathrm{PH})$ and its mechanism in patients with borderline hemodynamic constellation. We evaluated the impact of a volume challenge (contrast) on hemodynamics and its prognostic implications in patients with severe aortic stenosis (AS) undergoing left and right heart catheterization prior to aortic valve replacement (AVR).

Methods: Mean pulmonary artery pressure (mPAP) and mean pulmonary artery wedge pressure (mPAWP) were measured in
285 patients with severe AS (indexed aortic valve area $0.41 \pm 0.13$ $\mathrm{cm}^{2} / \mathrm{m}^{2}$, left ventricular ejection fraction $58 \pm 12 \%$ ) before and immediately after administration of $150 \pm 58 \mathrm{ml}$ of low or isoosmolal contrast. Mortality after a median follow-up of 1310 days post-AVR was assessed.

Results: Pre-contrast mPAP and mPAWP were $25 \pm 10$ and $16 \pm 8 \mathrm{mmHg}$. Before contrast $112(39 \%)$ patients had PH: 98 with post-capillary [63 with isolated post-capillary $\mathrm{PH}(\mathrm{IpcPH}), 35$ with combined pre- and post-capillary $\mathrm{PH}(\mathrm{CpcPH})], 14$ with pre-capillary $\mathrm{PH}$. There was a post-contrast rise in $\mathrm{MPAP}$ and $\mathrm{mPAWP}$ by $5 \pm 4$ and $4 \pm 4 \mathrm{mmHg}$ to $30 \pm 11$ and $20 \pm 8 \mathrm{mmHg}$ resulting in 70 additional patients with $\mathrm{PH}$ (40\% of those without pre-contrast $\mathrm{PH}$ ): 61 with post-capillary, 9 with pre-capillary $\mathrm{PH}$. Patients with pre-contrast $\mathrm{PH}$, post-contrast $\mathrm{PH}$ only, and those without any $\mathrm{PH}$ are compared in the Table. There was a trend towards higher mortality in patients with pre-contrast $\mathrm{PH}$ vs. no pre-contrast $\mathrm{PH}$ (Figure 1A), which was driven by $\mathrm{CpcPH}$ patients (Figure 1B). However, patients with post-contrast $\mathrm{PH}$ only had similar mortality as patients with no $\mathrm{PH}$ (Figure 1C), and post-contrast changes in 
Table 1. (for Abstract 120)

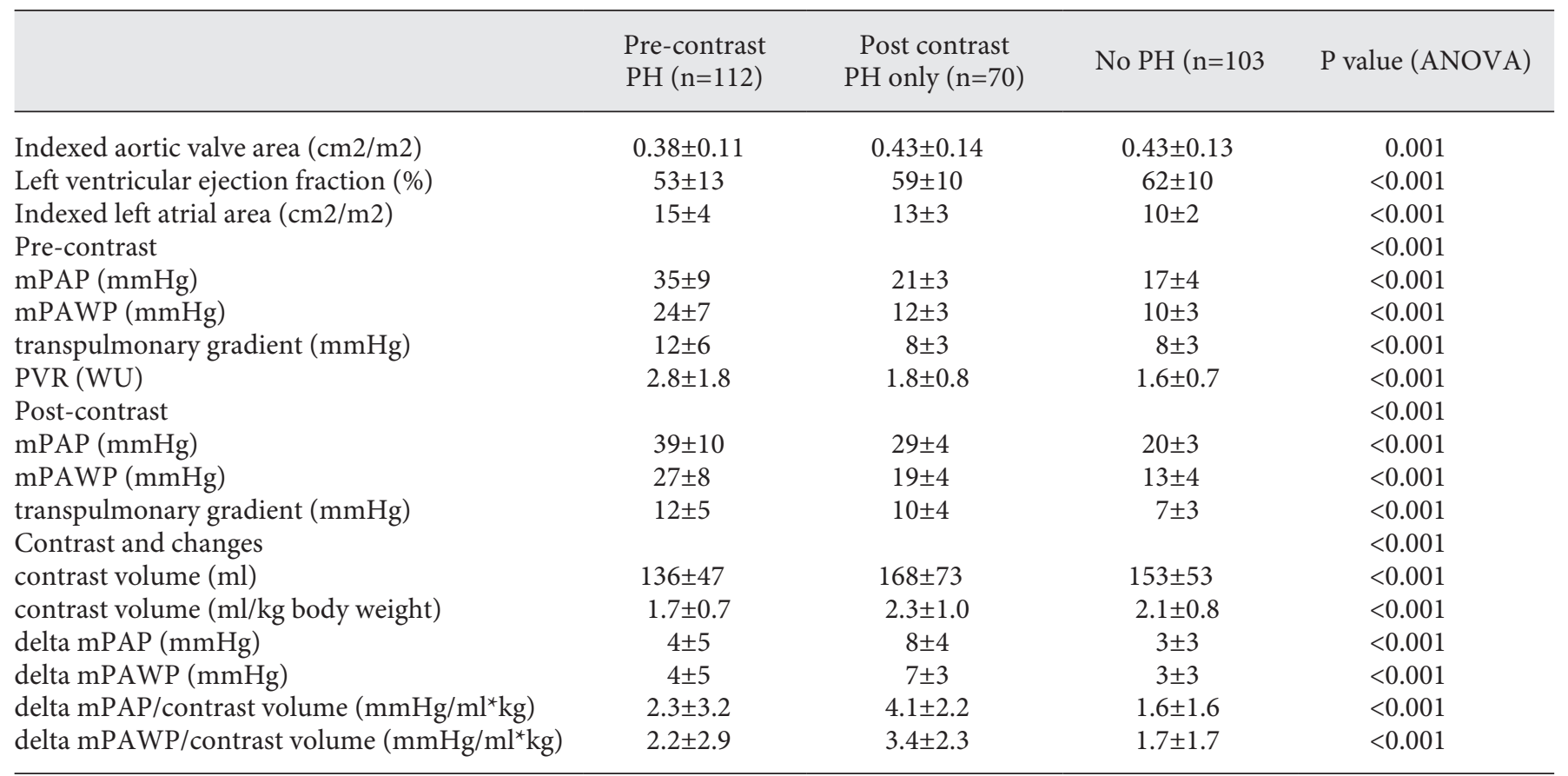

A

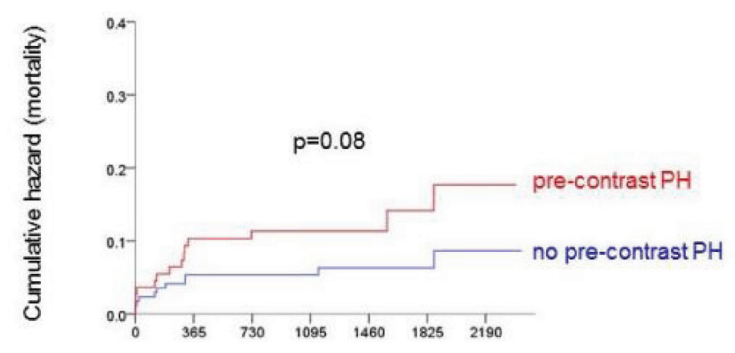

B

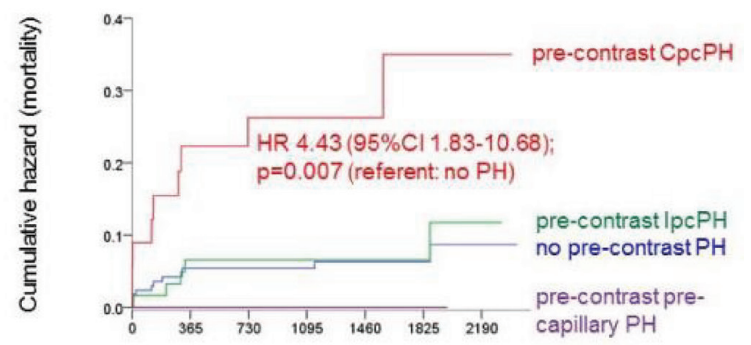

C

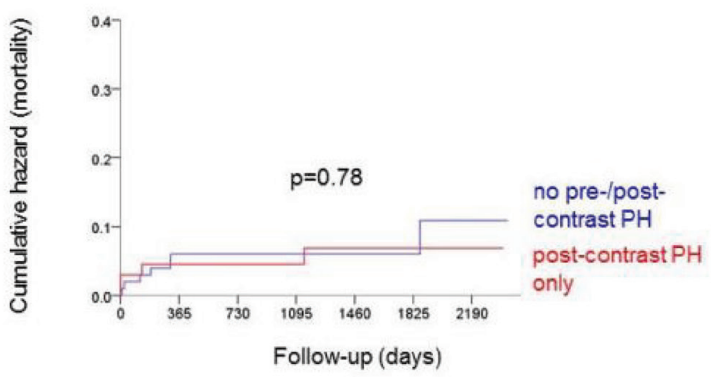

Fig. 1. (for Abstract 120)
mPAP and mPAWP were not associated with mortality (data not shown).

Conclusions: In patients with severe AS, contrast administration leads to post-contrast PH in approximately $40 \%$ of patients without pre-contrast $\mathrm{PH}$. However, in contrast to the pre-contrast constellation post-contrast changes seem to be of limited prognostic value.

Disclosure: Nothing to disclose

\section{1 \\ Novel Definition of Pulmonary Hypertension: Impact on Classification and Prognosis in Patients with Severe Aortic Stenosis}

M.T.Maeder ${ }^{1}$, L. Weber ${ }^{1}$, D. Weilenmann ${ }^{1}$, J. Chronis ${ }^{2}$, L. Joerg ${ }^{1}$, S. Pohle , P.K. Haager ${ }^{1}$, M. Brutsche ${ }^{1}$, O.D. Schoch ${ }^{1}$, H. Rickli ${ }^{1}$

${ }^{1}$ Kantonsspital St. Gallen, St. Gallen, Switzerland, ${ }^{2}$ Kantonsspital St. Gallen, St. Gallen, Sweden

Introduction: The $20186^{\text {th }}$ World Symposium on Pulmonary Hypertension $(\mathrm{PH})$ has proposed a novel $\mathrm{PH}$ definition including 1) a lower mean pulmonary artery pressure (mPAP) cut-off $(>20$ $\mathrm{mmHg}$ versus $\geq 25 \mathrm{mmHg}$ ) and 2) compulsory requirement of pulmonary vascular resistance (PVR) $\geq 3$ Wood units (WU) for definition of pre-capillary $\mathrm{PH}$. We assessed the clinical impact of this novel definition in patients with aortic stenosis (AS).

Methods: 487 patients with severe AS (indexed aortic valve area $0.41 \pm 0.13 \mathrm{~cm}^{2} / \mathrm{m}^{2}$, left ventricular ejection fraction $58 \pm 12 \%$ ) 
Table 1. (for Abstract 131)

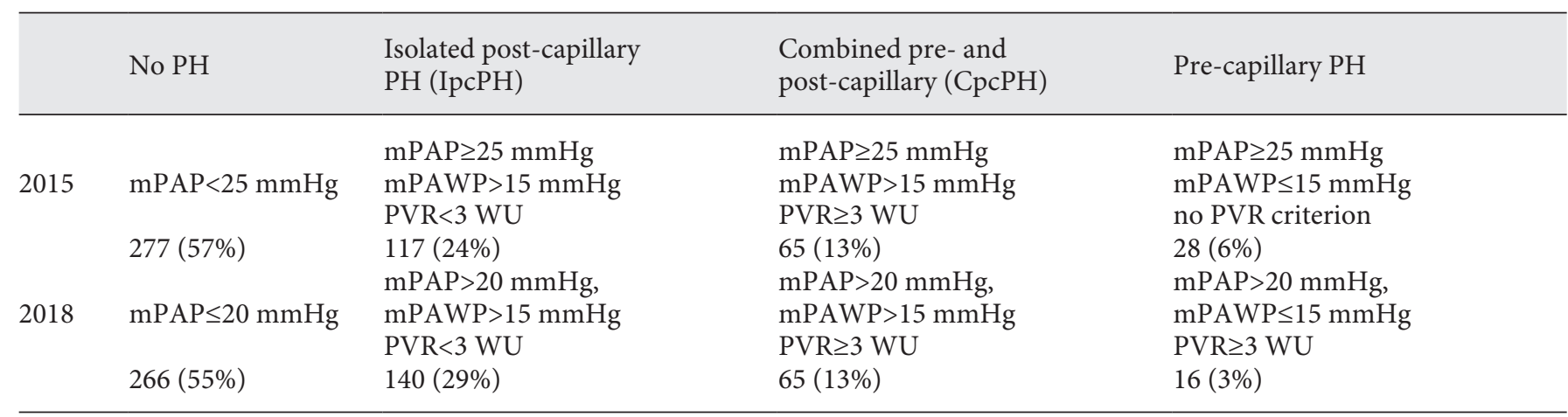

\section{A 2015 Definition}

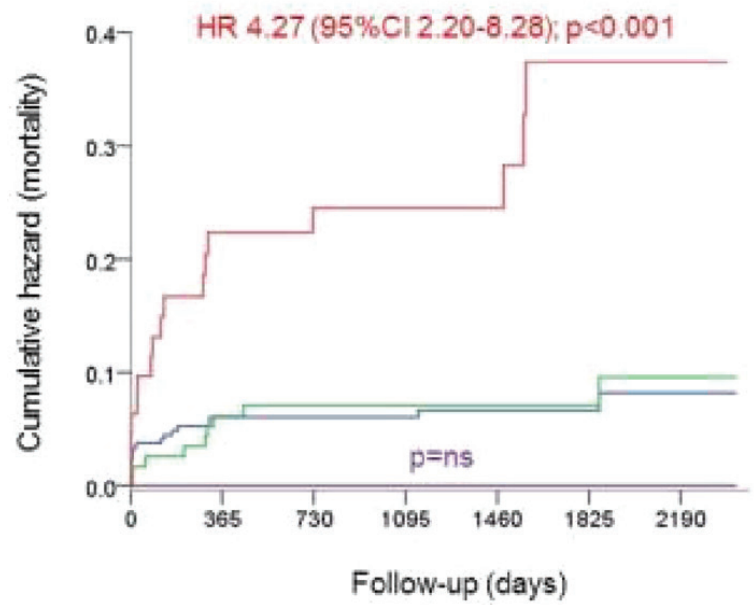

Fig. 1. (for

Abstract 131)

\section{B 2018 Definition}

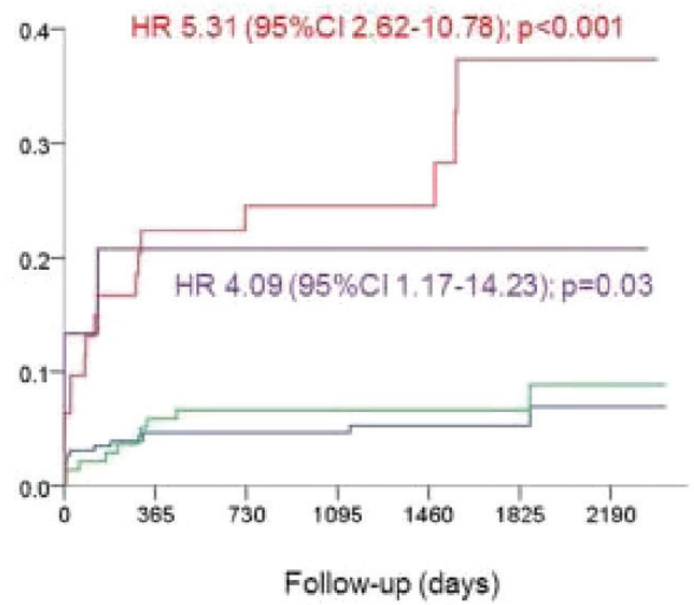

No $\mathrm{PH}$ (referent)
IpcPH
CpcPH
Pre-capillary PH undergoing right heart catheterization prior to valve replacement (AVR) were classified according to the current $2015 \mathrm{PH}$ definition and the 2018 proposal (Table). Post-AVR mortality [median follow-up 1345 (951-1883) days] was assessed.

Results: Overall mPAP, mPAWP, and PVR were $25 \pm 10 \mathrm{mmHg}$, $16 \pm 8 \mathrm{mmHg}$, and $2.1 \pm 1.3 \mathrm{WU}$ respectively. Classification according to the 2015 and 2018 definitions is shown in the Table. 51(11\%) patients were reclassified: no $\mathrm{PH}$--> isolated post-capillary $\mathrm{PH}(\mathrm{IpcPH}): \mathrm{n}=23$, no $\mathrm{PH}-->$ precapillary $\mathrm{PH}: \mathrm{n}=8$, pre-capillary $\mathrm{PH}-->$ no $\mathrm{PH}: \mathrm{n}=20$ [no changes from or to a diagnosis of combined pre- and post-capillary $\mathrm{PH}(\mathrm{CpcPH})]$. According to the 2015 definition, only $\mathrm{CpcPH}$ patients had higher mortality than patients without $\mathrm{PH}$ (Figure 1A). In contrast, when applying the
2018 definition patients with both $\mathrm{CpcPH}$ and pre-capillary $\mathrm{PH}$ had higher mortality than those without $\mathrm{PH}$ (Figure 1B). Among the $\mathrm{PH}$ definition components, $\mathrm{PVR} \geq 3 \mathrm{WU}$ was the strongest predictor of death (Figure 2: hazard ratio of more than 4 vs. approximately 2 for the two mPAP cut-offs and mPAWP).

Conclusions: In severe AS, the novel PH definition diagnoses more patients with IpcPH who however have similar prognosis as those without $\mathrm{PH}$. Patients with true pre-capillary $\mathrm{PH}$ are better identified by the 2018 proposal by proof of presence of pulmonary vascular disease by $P V R \geq 3 \mathrm{WU}$, which is a strong predictor of poor prognosis.

Disclosure: Nothing to disclose 
A

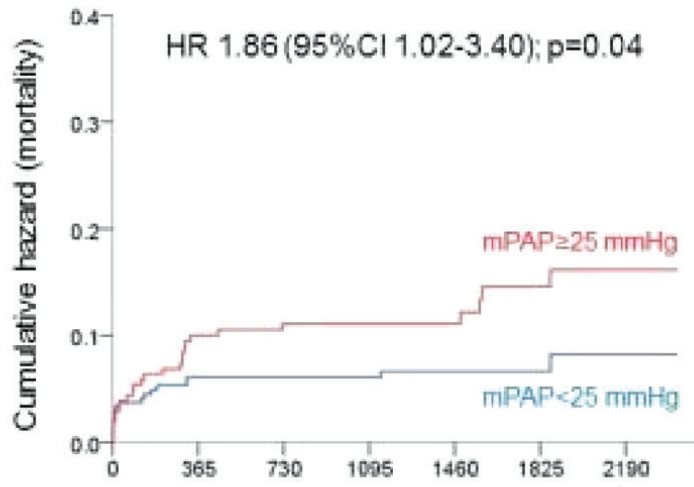

Fig. 2. (for

Abstract 131)

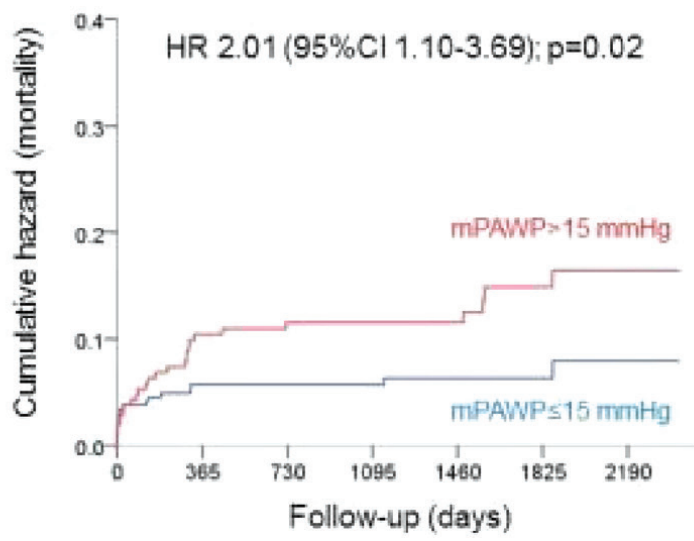

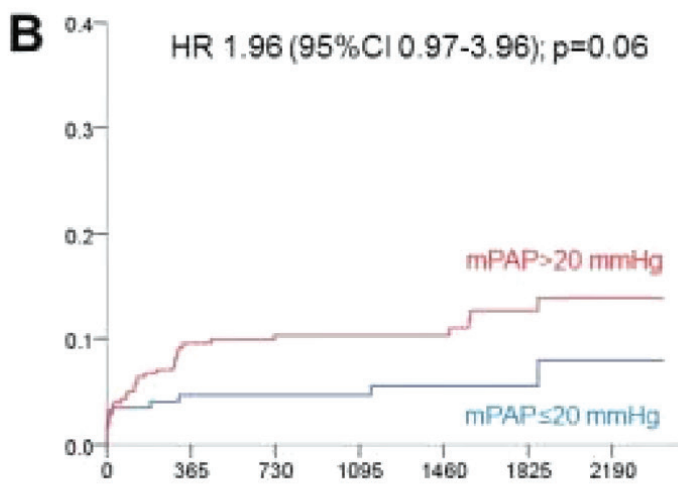

D

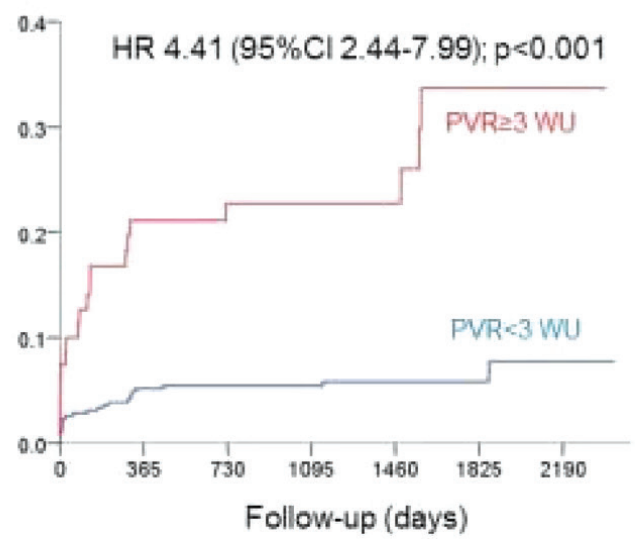

\section{5}

\section{Effect of Normobaric Hypoxia on Constant Work- Rate Cycle Time in Patients with Precapillary Pulmonary Hypertension - A Randomized Crossover Trial}

\section{S.R. Schneider $^{1,2}$, L.C. Mayer ${ }^{1}$, M. Lichtblau', C. Berlier', E.I. Schwarz', S. Saxer ${ }^{1}$, M. Furian' , K.E. Bloch'1, S. Ulrich ${ }^{1}$ \\ ${ }^{1}$ Pulmonology, University Hospital Zurich, Zürich, ${ }^{2}$ Health Sciences and Medicine, University of Lucerne, Lucerne, Switzerland}

Introduction: Patients with pulmonary arterial or chronic thromboembolic pulmonary hypertension $(\mathrm{PH})$ who are characterised by exercise desaturation wish to travel to altitude, but hypoxia-related adverse health effects are insufficiently explored. We investigated how normobaric hypoxia (hypoxia) affects constant work-rate exercise time (CWRET) as well as cardiac and respiratory physiology compared to ambient air (placebo) in $\mathrm{PH}$.

Methods: In a randomized crossover trial, stable $\mathrm{PH}$-patients with a $\mathrm{PaO}_{2}>7.3 \mathrm{kPa}$ were assigned to hypoxia $\left(\mathrm{FiO}_{2} 15 \%\right)$ vs. placebo $\left(\mathrm{FiO}_{2} 21 \%\right)$ for $\geq 20$ ' at rest and during a symptom-limited cycle CWRET ( $60 \%$ Wmax). The primary outcome was the change in CWRET in hypoxia compared to placebo. Secondary outcomes were changes in symptoms, blood- and tissue oxygenation and echocardiography.

Results: 28 patients (46\% women), median (quartiles) age $66(54 ; 74)$ years, mean pulmonary artery pressure $41(29 ; 49) \mathrm{mmHg}$ were included. Overall, CWRET was significantly lower during hypoxia vs. placebo $(6.7(5.5 ; 27.3)$ vs. $16.9(8.0 ; 30.0) \mathrm{min}, \mathrm{p}=0.006)$ along with increased VAS-dyspnoea $(6.6(1.8 ; 8.7)$ vs. $4.8(2.0 ; 7.2)$ $\mathrm{cm}, \mathrm{p}=0.036)$ Borg-CR10 leg fatigue $(5(3 ; 8)$ vs. $6(3 ; 8), \mathrm{p}<0.001)$. $\mathrm{PaO}_{2}$ was reduced with hypoxia: $8(7.3 ; 9.9)$ vs. $6.4(5.4 ; 7.9) \mathrm{kPa}$, $\mathrm{p}=0.003$, whereas echocardiography and other parameters at rest and peak CWRET were unchanged. No serious adverse events occurred.

Conclusion: During a short exposure to moderate hypoxia (altitude equivalent of $2500 \mathrm{~m}$ ), patients with $\mathrm{PH}$ experienced a slight reduction in CWRET associated with a greater degree of dyspnea and leg fatigue while hemodynamics assessed by echocardiography and other assessments remained unchanged.

Disclosure: Nothing to disclose 


\section{Thoracic Surgery}

38

\section{Uniportal Lingula-Sparing Left Upper Lobectomy for Swyer-James-Macleod Syndrome}

\author{
G.J.Kocher ${ }^{1}$, A. Zehnder ${ }^{2}$ \\ ${ }^{1}$ Thoracic Surgery, ${ }^{2}$ Bern University Hospital and University of \\ Bern, Bern, Switzerland
}

Introduction: Swyer-James-MacLeod syndrome is a rare entity and management generally consists of conservative measures in the form of bronchodilatators as well as early treatment of infections.

Methods: We herein report the management of an unusual case of Swyer-James-MacLeod Sydrome in a 37-year old male patient. The patient was referred with continuously worsening dyspnea over the past two years. On admission he suffered from severe dyspnea on mildest exertions and even when speaking. CT-scan showed a nearly complete emphysematous destruction of the left lung with hyperinflation and mediastinal shift to the right side. The insertion of endobronchial valves was evaluated by the pneumologists and finally rejected because of the very narrowed central bronchi and only little visible remaining viable lung tissue on the left on CT-scan.

Results: Surgical exploration via left uniportal VATS was performed and revealed that only the apical three segments of the left lung were destroyed and emphysematic so that they compressed the remaining viable lung on the left. Surgical treatment therefore consisted of a lingula-sparing upper lobectomy, saving the lingula and the lower lobe. Operative time was 240 minutes and the postoperative course was uneventful. Before Hospital discharge one week after surgery, the patient was already able to walk 16 flights of stairs in one go.

Conclusion: In the presented case the relatively rapidly progressing dyspnea of the patient, despite bronchodilatator treatment, prompted us to evaluate a surgical treatment approach. Fortunately for the patient we were able to carry out a minimally invasive procedure and save the lingula as well as the left lower lobe. In selected cases of SJM-syndrome surgical resection may be a good treatment option in case conservative measures fail to control the disease.

Disclosure: Nothing to disclose

\section{9}

\section{Approach to Primary Spontaneous Pneumothorax by Surgeons: A National Survey of Current Swiss Practice}

\section{B. Bédat, M. Demarchi, F. Triponez, W. Karenovics}

Thoracic and Endocrine Surgery, University Hospital Geneva, Geneva, Switzerland

Pneumothorax is a common pathology, but optimal management strategies are not yet defined. There are significant differences in international guidelines and therefore variation in clinical practice. We aimed to determine the current surgical practice in Switzerland.

An online questionnaire regarding management, indications and surgical strategy was sent to one surgeon in all thoracic units, including secondary and tertiary hospitals, and preferably to members of the Swiss Society of Thoracic Surgery. Questionnaire was sent in French, German and in Italian.

Fifteen surgeons over twenty-eight (54\%), mainly thoracic, responded to the survey. A majority of surgeons (73\%) consider that they follow international guidelines rather faithfully. Concerning the indication of drainage, $60 \%$ of surgeons used criteria of more than one guidelines. The guidelines used are the British Thoracic Society guidelines (73\%), the American College of Chest Physician guidelines (53\%), the Belgian Society of Pneumology (27\%), the S3-Leitlinie guidelines (7\%). To drain the pneumothorax, one center used needle aspiration, six used small bore drains ( $£ 14 \mathrm{Fr})$ and eight used large bore drains ( $>14 \mathrm{Fr})$. Currently, most surgeons (87\%) use digital drains to monitor air leaks, and two centers used a Heimlich valve. Four centers performed computed tomography after the first episode of pneumothorax and four centers after the second episode. Video-assisted thoracic surgery was unanimously the preferred approach. Regarding the timing of surgery, $1(7 \%)$ surgeon would intervene at first presentation, $12(80 \%)$ for a first bilateral pneumothorax, 10 (67\%) for a tension pneumothorax, 11 (73\%) for persistent lung collapse, 4 (27\%) for evidence of bullae $>2 \mathrm{~cm}$, and 11 (73\%) for persistent air leak, with a median of 4 days (range of 3-7 days). Fourteen surgeons (93\%) performed an apical bullectomy. Concerning pleurodesis, pleural abrasion was the preferred technique for 10 surgeons (67\%), chemical pleurodesis and pleurectomy was the choice for 2 surgeons respectively, and partial pleurectomy+pleural abrasion was performed by one surgeon. Surgeons perceived recurrence rates at $1 \%-10 \%$.

There is variability in the management of primary spontaneous pneumothorax among surgeons across Switzerland. New trials are required to improve a personalize management.

Disclosure: Nothing to disclose 


\section{1 \\ Release of Damage-Associated Molecular Patterns (DAMPs) and Cytokines by Lung Grafts in an Experimental Model of EVLP and Lung Transplantation}

A. Hasenauer ${ }^{1}$, E. Abdelnour-Berchtold' ${ }^{1}$, B. Bédat ${ }^{2}$, R. Parapanov ${ }^{1}$, A. Debonneville ${ }^{1}$, M. Gonzalez' ${ }^{1}$ J.-Y. Perentes ${ }^{1}$, J. Lugrin ${ }^{1}$, L. Piquilloud ${ }^{1}$, L. Liaudet ${ }^{1}$, T. Krueger ${ }^{1}$

${ }^{1}$ Lausanne University Hospital (CHUV), Lausanne, ${ }^{2}$ Geneva University Hospitals, Genève, Switzerland

Objectives: Shortage of donor organs limits the access of patients to lung transplantation (LTx). Ex vivo lung perfusion (EVLP) has emerged as a technique permitting to increase the number of lungs available for LTx. An important but poorly described effect of EVLP is the induction of inflammatory changes in the donor lung. The underlying mechanisms and consequences in terms of transplant suitability are largely unknown. Here we assess if the reoxygenation of the ischemic lung during EVLP induces inflammatory pathways in an experimental animal model and its effect on the physiological function of the lung after LTx.

Methods: Using a rodent model of lung procurement, normothermic EVLP and left single lung transplantation, 3 experimental groups (each group $\mathrm{n}=6$ ) were assessed : Donor lungs were 1) preserved at $4^{\circ} \mathrm{C}$ for $7 \mathrm{~h}$ (group cold ischemia (CI)), or 2) preserved for $1 \mathrm{~h}$ at room temperature and $6 \mathrm{~h}$ at $4^{\circ} \mathrm{C}$ (group warm ischemia (WI)), or 3) were preserved for $1 \mathrm{~h}$ at room temperature, underwent $3 \mathrm{~h}$ EVLP and kept inflated $3 \mathrm{~h}$ at $4^{\circ} \mathrm{C}$ (group $\mathrm{WI}_{\mathrm{E}}$ ). The left lung was transplanted into a recipient rat and after 2 hours of in situ blood reperfusion, cytokines, DAMPs and cell damage biomarkers were measured in the bronchoalveolar lavage (BAL). Pulmonary compliance was also evaluated in-vivo.

Results: Compared to CI, donor lung exposure to WI induced more pronounced lung damage $(\mathrm{p}<0,05)$, increased inflammatory cytokines $(p<0,05)$, increase in DAMP HMGB1 $(p<0,05)$ and decreased pulmonary compliance. EVLP in lungs exposed to WI was associated with reduced lung damage $(\mathrm{p}<0,05)$, increased inflammatory cytokines $(\mathrm{p}<0,05)$, increased pulmonary compliance, but no effect on DAMPs release.

\section{Conclusion:}

1) EVLP leads to inflammation which is not correlated with lung function.

2) Major DAMPs are released after warm ischemia with no influence of EVLP.

3) EVLP on damaged lungs reduces lungs damages and graft dysfunction.

Disclosure: Nothing to disclose

\section{8 \\ Open Chest Management after Cardiac Surgery: In-Hospital Outcomes and Factors Affecting Mortality}

\author{
V. Ntinopoulos, S. Dushaj, A. Haeussler, N. Papadopoulos, \\ D. Odavic, H. Loeblein, L. Rings, D. Brugnetti, O. Dzemali
}

Cardiac Surgery Department, Triemli Hospital, Zurich, Switzerland

Introduction: Open chest management is an important treatment option for hemodynamically unstable or severely bleeding patients who cannot tolerate primary sternal closure after cardiac surgery. Even though associated with bad postoperative outcomes, few data exist about factors affecting mortality. Aim of this study is to evaluate in-hospital outcomes and factors affecting mortality in patients undergoing open chest management after cardiac surgery.

Methods: We performed a retrospective analysis of the data of patients undergoing open chest management in our institution in the period 05/2008-08/2019. All the patients underwent open chest management because of hemodynamic instability or severe coagulopathy after cardiac surgery with inability to perform primary sternal closure. In-hospital outcomes were evaluated and perioperative factors affecting mortality were assessed.

Results: Patients undergoing open chest management exhibited a high rate of deep sternal wound infection (10.8\%), postoperative reanimation $(28.4 \%)$, kidney replacement therapy (63.5\%) and a high in-hospital mortality (56.8\%). Patients who did not survive open chest management had a statistically significantly older age ( 78.5 vs 73.5 years, $\mathrm{p}=0.04$ ), longer cumulative cardiopulmonary bypass duration at the index operation (224 vs. 161 minutes, $\mathrm{p}=0.031$ ), higher incidence of arterial hypertension ( 65.4 vs $34.6 \%, \mathrm{p}=0.006)$ and higher incidence of reanimation postoperatively (76.2 vs $23.8 \%$, $\mathrm{p}=0.034$ ) in comparison to survivors.

Conclusion: Open chest management is associated with high in-hospital morbidity and mortality. Perioperative factors associated with higher in-hospital mortality are older age, longer cumulative cardiopulmonary bypass duration at the index operation, presence of arterial hypertension and postoperative reanimation.

Disclosure: Nothing to disclose 


\section{9}

\section{Active Clearance of Chest Tubes after Cardiac Surgery: A Propensity Score Matched Analysis}

V. Ntinopoulos ${ }^{1}$, A. Haeussler ${ }^{1}$, N. Papadopoulos ${ }^{1}$, D. Odavic ${ }^{1}$, P. Fodor ${ }^{2}$, D. Brugnetti ${ }^{1}$, L. Rings ${ }^{1}$, S. Dushaj ${ }^{1}$, O. Dzemali ${ }^{1}$

${ }^{1}$ Cardiac Surgery Department, ${ }^{2}$ Department of Anesthesiology and Intensive Care Medicine, Triemli Hospital, Zurich, Switzerland

Introduction: Chest tubes inserted to evacuate shed mediastinal blood after cardiac surgery often become clogged, limiting their capacity to evacuate blood, leading to blood retention. In this propensity score matched study, we evaluate a method of active tube clearance (ATC).

Methods: This study included 2461 adult patients undergoing cardiac surgery. Patients receiving conventional chest tubes only ( $n=1980$ ) were compared with patients receiving an ATC-tube in retrosternal position $(\mathrm{n}=481)$ for chest tube output, re-exploration for bleeding or tamponade and interventions for pleural effusion or pneumothorax, kidney replacement therapy, postoperative atrial fibrillation and sternal infection, before and after propensity score matching.

Results: Propensity score matching, generated 471 patientpairs balanced for their baseline characteristics. Matched patients with ATC had less chest tube output at 24 hours (590 [380-905] ml vs 480 [316-700] $\mathrm{ml}, \mathrm{p}<0.0001)$ und 48 hours $(597$ [383-962] ml vs 505 [342-800] ml, p =0.0001) postoperatively. Intervention for pleural effusion was not different between matched patients $(28 \%$ vs $28 \%, \mathrm{p}=1)$. Patients with ATC had a lower rate of reexploration ( $4 \%$ vs $2.5 \%, p=0.25)$, intervention for pneumothorax (4.9\% vs $4.7 \%, \mathrm{p}=1)$, hemofiltration ( $5.7 \%$ vs $4.5 \%, \mathrm{p}=0.45)$, postoperative atrial fibrillation $(22 \%$ vs $19 \%, \mathrm{p}=0.33)$ and sternal infection $(0.64 \%$ vs $0.21 \%, p=0.62)$, although not statistically significant.

Conclusion: An ATC-tube in retrosternal position reduced chest tube output and showed a tendency towards reduction in the rate of reexploration for bleeding or tamponade, intervention for pneumothorax, kidney replacement therapy, postoperative atrial fibrillation and sternal infection.

Disclosure: Nothing to disclose

\section{6 \\ To Assess Whether 2-Octyl Cyanoacrylate Skin Adhesive (DERMABOND TM Topical Skin Adhesive, Ethicon) is the Ideal Wound Closure Technique in Cardiac Surgery Limiting Surgical Site Infection}

Z. Gunga ${ }^{1}$, M. Kirsch ${ }^{2}$

${ }^{1}$ Cardiac Surgery, CHUV, ${ }^{2}$ CHUV University Lausanne, Lausanne, Switzerland

Introduction: Surgical site infection (SSI) is a major source of morbidity after cardiac surgery procedures resulting in prolonged inpatient stay because long term intravenous antibiotics, re-operation or re-admission. Together with the procedure, wound closure is of utmost importance in cardiac surgery with however the presence of major limitations in the current methods of skin closure.Sutures and staples have been commonly used in our practice until we adopted the use of DERMABOND PRINEO skin closure system.

Objective: To evaluate the effects of the topical skin adhesive 2-octyl cyanoacrylate PRINEO (DERMABONDTM Topical Skin Adhesive, Ethicon), used as the final layer in sternotomy closure in patients undergoing cardiac surgery,on the incidence of postoperative surgical site infection

Methods:

-This is a retrospective observational cohort study including adult patients who underwent cardiac surgery between January 1 st 2015 and December 31st 2018 in our department.

-The exclusion criteria were patients who underwent heart transplantation and ventricular assist device operations, age less than 18.

-We devised 2 groups of patients by virtue of the type of wound closure technique

Group $1 \mathrm{n}=840$ : 2-octyl cyanoacrylate skin adhesive(PRINEO)

Group $2 \mathrm{n}=764$ : staples/steristrip under opsite dressing.

-The primary endpoint of our study is the occurrence of SSI : superficial infection (skin infection, subcutaneous infection) and deep infection (sternitis and mediastinitis).

Results: A total of 1604 patients was enrolled in our study with occurrence of 48 SSI during the time lapse.

The mean age was 63 years. Both groups were homogeneous in terms of the risk factors of mediastinis : diabetes mellitis (requiring insuline), obesity (BMI>30), smoking, renal impairment, length of surgery, mean age and sex.

The rate of SSI was found lower in group 1 (closure with Dermabond PRINEO), $\mathbf{1 , 9 0} \%$, compared to the other group, $4,19 \%$.

Group 1 was also superior in terms of lower length of hospital stay .

Furthermore, the subsequent decline in SSI after 2017, since the adoption of PRINEO, has been highlighted by the observation made by Swissnoso in their annual analysis of SSI.

Conclusion: DERMABOND Adhesive has proved, in our practice, to be a safe wound closure treatment that provides an ideal healing environment with its antimicrobial protection, reducing considerably surgical site infection and helps to reduce the economic burden of current care (frequent dressing changes).

Disclosure: Nothing to disclose 


\section{Paediatric}

74

Pulmonary Dysfunction after Treatment for Childhood Cancer - Comparing Multiple-Breath Washout with Spirometry

\author{
J. Usemann ${ }^{1,2}$, C. Schindera ${ }^{1,3}$, S. Zuercher ${ }^{4}$, R. Jung ${ }^{4}$, \\ R. Kasteler ${ }^{5}$, B. Frauchiger ${ }^{5}$, C.S. Rueegg ${ }^{6}$, P. Latzin ${ }^{5}$, C.E. Kuehni ${ }^{3}$, \\ N.X. von der Weid ${ }^{1}$ \\ ${ }^{1}$ University Children's Hospital Basel (UKBB), Basel, ${ }^{2}$ University \\ Children's Hospital Zurich, Zurich, ${ }^{3}$ Institute of Social and \\ Preventive Medicine, Bern, ${ }^{4}$ Epidemiology, Biostatistics \\ and Prevention Institute, Zurich, ${ }^{5}$ Bern University Hospital \\ and University of Bern, Ben, Switzerland, ${ }^{6}$ Oslo Centre for \\ Biostatistics and Epidemiology, Oslo, Norway
}

Rationale: Childhood cancer survivors are at risk of long-term pulmonary dysfunction, but we lack sensitive outcome measures to detect early pulmonary damage.

Objective: To assess the ability of nitrogen multiplebreath washout $\left(\mathrm{N}_{2} \mathrm{MBW}\right)$ for detecting pulmonary dysfunction compared to spirometry in long-term childhood cancer survivors.

Methods: Cross-sectional analysis from long-term ( $\geq 5$-year) childhood cancer survivors. We categorized survivors by risk: i) high risk: pulmotoxic chemotherapy, chest radiation, thoracic surgery, and/or hematopoietic stem cell transplantation ii) standard risk: other cancer therapies. Primary outcomes were lung clearance index (LCI), acinar ventilation inhomogeneity index $\left(\mathrm{S}_{\mathrm{ACIN}}\right)$, and forced expiratory volume in one second $\left(\mathrm{FEV}_{1}\right)$ as well as forced vital capacity (FVC). We calculated $z$ scores and compared pulmonary dysfunction between risk groups.

Results: We studied 46 survivors, median age at diagnosis 10 years, and 30 years at study. $37 \%$ were at the high, $63 \%$ in the standard risk group. LCI and $\mathrm{S}_{\mathrm{ACIN}}$ were higher in the high risk vs the standard risk group (mean LCI 2.09, standard deviation [SD] 2.39 vs 0.95 , SD 2.81; mean $\mathrm{S}_{\text {ACIN }} 2.45$, SD 3.29 vs 0.65 , SD 2.79). $\mathrm{FEV}_{1}$ and $\mathrm{FVC}$ were lower in the high risk vs the standard risk group (mean $\mathrm{FEV}_{1}-0.94$, SD 1.39 vs -0.10 , SD 1.07; mean FVC -1.14 , SD 1.23 vs 0.15 , SD 1.61). LCI, $\mathrm{S}_{\mathrm{ACIN}}, \mathrm{FEV}_{1}$, and FVC were abnormal in $60 \%, 53 \%, 33 \%$, and $33 \%$ of high risk patients compared to $23 \%, 21 \%, 0 \%$, and $4 \%$ of standard risk patients.

Conclusion: $\mathrm{N}_{2} \mathrm{MBW}$ identified more cases of pulmonary dysfunction in long-term childhood cancer survivors than spirometry, even in patients with cancer therapy not known as pulmotoxic. $\mathrm{N}_{2} \mathrm{MBW}$ could be an effective screening tool for early pulmonary damage after childhood cancer treatment.

Disclosure: Nothing to disclose

\section{6 \\ Description of Potential Presymptomatic Markers for Early Detection of Cardiopulmonary Complications in Pediatric Patients with Sickle Cell Disease}

H. Giron ${ }^{1}$, M. Beck Popovic ${ }^{2}$, I. Rochat ${ }^{3}$

${ }^{1}$ Faculty of Biology and Medicine, University of Lausanne,

2Pediatric Hemato-Oncology/ Woman Mother Child

Department, ${ }^{3}$ Pediatric Pulmonology/ Woman Mother Child

Department, Lausanne University Hospital (CHUV) and

University of Lausanne, Lausanne, Switzerland

Sickle cell disease (SCD), characterized by abnormal hemoglobin production, is a common genetic disease concerning millions of individuals worldwide. The first signs can appear early in life and affect every organ.

Acute pulmonary complications (acute chest syndrome, ACS) or chronic (loss of lung function, pulmonary hypertension) are common and may be life-threatening or limiting. ACS is a potentially fatal event presenting with acute chest pain, fever, respiratory symptoms and radiological abnormalities. Recurrent episodes of ACS can result in chronic lung disease and are linked to premature mortality.

In order to prevent ACS and improve management of pulmonary complications, predictors and specific markers are needed. Currently, clinical and hematological risk factors for ACS (infections, associated asthma, hemolysis) cannot be used for screening.

There have been recent reports of the role of circulating biological markers indicating impending ACS or future cardiopulmonary complications. For example, inflammatory markers such as CRP, sPLA2 and PTX3, though non-specific for ACS, are elevated prior to such an acute event. Likewise, interleukin-6, platelet derived growth factor (PDGF-BB) and vascular endothelial growth factor (VEGF) are strongly correlated with cardiac dysfunction. Nitric oxide (NO), an important pathway of vasodilatation, is significantly reduced in SCD patients, whereas Fas and its ligand FasL, members of the tumor necrosis factor receptor superfamily, are significantly higher in SCD patients compared to healthy controls and SCD patients treated with hydroxyurea. Finally, low levels of serum Apelin, a peptide involved in cardiovascular function, seem to be present in early stage subclinical cardiac disease and could enable the early recognition of high risk patients. Thus these biomarkers may possibly help identify early vascular damage and dysfunction.

This review describes potentially useful biological biomarkers of cardio-pulmonary complications in SCD in order to develop strategies for their early detection at a subclinical stage and proposes new guidelines for follow-up.

Disclosure: Nothing to disclose 


\section{8}

\section{Recurrent Pneumonia in a 15 Year-Old Patient: What could be the Underlying Cause?}

\section{A. Kuhn, D. Müller-Suter, P. Eng}

Pediatric Pulmonology and Allergy/Clin. Immunology, Kantonsspital Aarau, Children's Hospital, Aarau, Switzerland

Introduction: A 15-year old male patient had been referred to pediatric pneumology due to three episodes of left-sided pneumonia within one year. At the age of nine he acquired pneumonia for the first time, followed by bursitis calcanei at the age of 13 and lymphangitis aged 14, otherwise no significant medical history. $\mathrm{He}$ had been asymptomatic between the episodes of pneumonia and had received vaccinations according to Swiss standard recommendations. Weight and length development was normal.

Methods: Bodyplethysmography showed normal results and chest $\mathrm{x}$-ray indicated no major abnormalities. Blood results revealed very low levels of $\operatorname{IgG}(0,03 \mathrm{~g} / \mathrm{l})$ and $\operatorname{IgA}(<0,06 \mathrm{~g} / \mathrm{l})$ and normal levels of IgM $(1,99 \mathrm{~g} / \mathrm{l})$. No tetanus-IgG antibodies could be found despite vaccination. Normal CBC including normal levels of leucocytes and lymphocytes. Subsequent differentiation of lymphocytes showed normal numbers of T-lymphocytes with slightly decreased amount of B-lymphocytes. CD8+ lymphocytes were not decreased, whereas low levels of memory-B-cells, plasmablasts and CD21low-B-cells could be detected.

Results: These blood results led to the diagnosis of common variable immunodeficiency (CVID). However, CD40-ligand-deficiency was not ruled out so far, but would not lead to acute change in treatment. A following CT scan of the lung showed mild bronchiectasis. Additional autoimmune diseases could not be detected. A regular substitution of immunoglobulins, physiotherapy and inhalation was started.

Conclusion: Inherited immunodeficiencies do not always present at very young age. CVID is a heterogeneous syndrome of primary immunodeficiency almost always associated with pulmonary complications, typically commencing in mid adolescence to young adulthood. Patients with recurrent major infections such as pneumonia, meningitis etc. or an abnormal amount of minor infections (i.e. otitis) should be tested for antibody deficiency. In case of confirmation of the diagnosis patients should receive regular immunoglobulin substitution and regular check-ups for pulmonary, oncological and autoimmune disease.

Disclosure: Nothing to disclose 


\section{Respiration}

Abdelnour-Berchtold E. 81

Abdraeva A.K. 173

Abu Hussein N. 191

Adler D. 1

Aeschbacher S.S. 73

Allali G. 1

Ammann P. 120

Anna-Barbara T. 159

Ardura-Garcia C. 269

Asisof A. 139

Assayag D. 166

Aydaralieva S. 173

Banz Y. 299

Bao Y. 111

Barata F. 15, 19

Baty F. 54, 75, 151, 256

Beck Popovic M. 176

Bédat B. 59, 81

Beigelman-Aubry C. 90

Berezowska S. 299

Berlier C. 255

Bernasconi E. 228

Bernasconi M. 90

Berra G. 1

Beyer S. 23, 153

Bhatt S. 166

Bitos K. 173, 263

Blank A. 299

Blank F. 159

Bloch K.E. 73, 173, 255, 263

Blumer S. 306

Bondarenko J. 166

Bongiovanni M. 227

Bösch M. 54

Bovet L. 166

Brack T. 75

Bremerich J. 261

Bridevaux P.O. 191

Bridevaux P.-O. 201

Brugnetti D. 98, 99

Brun P. 166

Brunschwiler T. 139

Brutsche M. 15, 19, 131, 151, 244, 256

Brutsche M.H. 54, 75

Bubendorf L. 261

Buenzli S. 173

Cadus C. 191

Camp P. 166

Cantero C. 5

Carta A.F. 263

\section{Author Index}

Numbers refer to Abstract numbers
Casutt A. 90, 207

Charbonnier F. 201

Charvoz L. 228

Chatelain S. 228

Chhajed P.N. 191

Chicherov V. 140, 201

Christe A. 207

Chronis J. 120, 131

Clarenbach C. 201, 244

Clarenbach C.F. 139, 155

Coulter F. 111

Daccord C. 207, 227, 228

Dalla Lana K. 32

De Boer K. 166

De Palma M. 43

Debonneville A. 81

Demarchi M. 59

Diamanti E. 207

Domon N. 228

Duchna H.-W. 201

Dupuis-Lozeron E. 1

Durham A.-D. 90

Dushaj S. 98, 99

Dzemali O. 98, 99

Egger J.-M. 129

Eng P. 258

Fang L. 205, 245, 306

Faouzi M. 207, 227

Ferrié C. 159

Filipin-Horvath V. 54

Fitting J.-W. 169, 171

Fleisch E. 15, 19

Flueck D. 73

Fodor P. 99

Franzen D. 111

Frauchiger B. 74

Frei A. 32

Furian M. 73, 173, 255, 263

Fytianos K. 159, 222

Garvey C. 166

Gauer J. 32

Gazdhar A. 159, 222, 270

Geiser T. 191, 222, 270

Geiser T.K. 236, 244

Geks A. 111

Giron H. 176

Gloeckl R. 166

Gonzalez M. 81

Goutaki M. 269
Grendelmeier P. 191, 261

Gross C.S. 139, 155

Guichard A. 43

Guler S.A. 166, 236, 244

Gumbiene L. 20

Gunga Z. 316

Gutweniger S. 73

Haager P.K. 120, 131

Haeussler A. 98, 99

Hage R. 167

Hagmann M. 256

Hambly N. 166

Hasenauer A. 81

Hasler E.D. 73

Hess T. 23

Hofer M. 23, 153

Holland A. 166

Hostettler K. 222, 244, 270

Hostettler K.E. 261

Huber F. 73

Humair G. 207

Hur S.A. 166

Ivankay A. 139

Jahn K. 261

Jarosch I. 166

Jochmann A. 201

Joerg L. 120, 131

Johannson K.A. 166

Jonigk D. 270

Joos Zellweger L. 191

Jung R. 74

Kähler C. 75

Karenovics W. 59

Karol A. 111

Kasteler R. 74

Keklikoglou I. 43

Khan P. 222, 270

Kinj R. 90

Kirsch M. 316

Kleger N. 111

Klein K. 111

Kleinsorge L. 191

Knudsen L. 222, 270

Kocher G.J. 38

Kohlbrenner D. 139, 155

Kohler M. 75, 191

Koller-Doser A. 129

Korobeynikova M. 140, 201

Kowatsch T. 15, 19
Krebser S. 87

Krempaska K. 244

Kreuter M. 166

Kronen P. 111

Krueger T. 81

Kuehnel M. 270

Kuehni C.E. 74, 269

Kuhn A. 258

Lambert N. 207

Langer R. 299

Latshang T.D. 73

Latzin P. 74, 269

Lawi D. 1

Lazor R. 207, 227

Lechartier B. 207

Lenoir A. 169, 171

Leroux J.-C. 111

Leuenberger C. 201

Leuppi J.D. 191, 201

Li J. 245

Liaudet L. 81

Lichtblau M. 73, 255, 263

Loeblein H. 98

Lovis A. 90, 170

Lugli A. 299

Lugrin J. 81

Lüthi-Corridori G. 191

Mademilov M. 173, 263

Maeder M.T. 120, 131

Maier S. 191

Makhdami N. 166

Mansouri N. 43

Marazhapov N.H. 263

Mayer L. 173

Mayer L.C. 255

McBride S.A. 166

Meier Laub L. 87

Mercier L. 228

Miedinger D. 191

Moeller A. 269

Morisset J. 166

Muller M.-E. 227

Müller-Suter D. 258

Mungo G. 23

Mutlu S. 159

Mykoniati S. 222

Myratbekova A. 173

Nassiri S. 43

Nicod L.P. 169, 171, 228

Nissen M. 139 
Noirez L. 90, 170

Ntinopoulos V. 98, 99

Odavic D. 98, 99

Ogna A. 228

Paciorkowski M. 151

Papadopoulos N. 98, 99

Parapanov R. 81

Paunović N. 111

Pedersen E.S.L. 269

Pendl G. 140, 201

Perentes J.-Y. 81

Perez Valdes C. 207

Pichler Hefti J. 87

Piquilloud L. 81

Plojoux J. 5

Pohle S. 131

Preisig M. 169, 171

Providoli R. 140

Puhan M. 15, 19, 32

Rassouli F. 15, 19, 54, 75

Rezek S. 23

Ribi C. 228

Rickli H. 120, 131

Rigger J. 120
Rings L. 98, 99

Riva M. 153

Rochat I. 176

Rohner F. 120

Roth M. 205, 245, 306

Rothe T. 201

Roux J. 270

Rüber F. 111

Rueegg C.S. 74

Ryerson C.J. 166

Savic Prince S. 261, 270

Saxer S. 20, 255

Schachner T. 155

Scheiwiller P.M. 73

Schilt C. 227

Schindera C. 74

Schliep R. 222

Schmid A. 299

Schneider S.R. 173, 255, 263

Schoch O.D. 131

Schuoler C. 140

Schuurmans M.M. 153, 167

Schwarz E.I. 255

Schwerzmann M. 87

Sheraliev U. 173, 263

Sievi N.A. 75
Similowski T. 1

Singer F. 269

Sooronbaev T.M. 173, 263

Steiner S. 159

Steurer-Stey C. 15, 19, 32, 140, 201

Stickland M.K. 166

Stolz D. 75, 205, 245, 261, 306

Strassmann A. 32

Strippoli M.-P.F. 169, 171

Studart A. 111

Sun K. 166

Svab S. 87

Tamm M. 75, 191, 205, 245, 261, 270, 306

Tan B. 166

Thurnheer R. 75

Tinschert P. 15, 19

Torchia B. 43

Touilloux B. 169,171

Triponez F. 59

Trippel M. 299

Tschacher A. 191

Tschirren U. 75

Turnheer R. 191
Ullmer E. 140

Ulrich S. $73,73,173$, 255,263

Urwyler P. 191

Usemann J. 74, 269

Vollenweider P. 169, 171

Von der Weid N.X. 74

Von Garnier C. 90, 170

Von Rechenberg B. 111

Vongthilath R. 5

Wald J. 166

Weber L. 120, 131

Weilenmann D. 120, 131

Wetzel S. 140, 201

Widmer S. 54, 75

Wohlfarth E. 236

Wotzkow C. 159

Zehnder A. 38

Zellweger A. 129

Zellweger J.-P. 129

Zhou L. 205

Zuercher S. 74 